\title{
DEVELOPMENT OF UAV-BASED HIGH-THROUGHPUT PHENOTYPING TECHNOLOGIES TOWARDS IMPROVING SOYBEAN BREEDING EFFICIENCY
}

\author{
A Dissertation presented to \\ the Faculty of the Graduate School at the \\ University of Missouri-Columbia \\ In Partial Fulfillment \\ of the Requirements for the Degree \\ Doctor of Philosophy \\ by \\ JING ZHOU \\ Dr. Jianfeng Zhou, Dissertation Supervisor \\ MAY 2021
}


(C) Copyright by Jing Zhou 2021

All Rights Reserved 
The undersigned, appointed by the dean of the Graduate School, have examined the dissertation entitled

\author{
DEVELOPMENT OF UAV-BASED HIGH-THROUGHPUT PHENOTYPING \\ TECHNOLOGIES TOWARDS IMPROVING SOYBEAN BREEDING EFFICIENCY \\ presented by Jing Zhou, \\ a candidate for the degree of doctor of philosophy of Biological Engineering, \\ and hereby certify that, in their opinion, it is worthy of acceptance.
}

Professor Jianfeng Zhou

Professor Gang Yao

Professor Leon Schumacher

Professor Henry T. Nguyen 


\section{DEDICATION}

To all my families and friends who supported, encouraged, and inspired me in the past 30 years of my life. 


\section{ACKNOWLEDGEMENTS}

The path to the dissertation is a unique experience full of challenges, sparks, achievements and fun. It would not have been completed without the supports from my advisor, collaborators, co-workers, and committee members. First, I would like to express my sincere appreciation to my academic advisor, Dr. Jianfeng Zhou for providing me the great opportunity of studying and working in the Precision and Automated Agriculture Lab (PAAL). From his work attitude and personal characteristics, I have learned to be a serious scholar but a humble man. His strong background in Engineering and rich experiences in working on agricultural problems led me to find meaningful research goals in plant phenotyping and form a systematic approach to reach the goals.

I would like to think my collaborators in the Department of Plant Science, Dr. Pengyin Chen and his crew Dr. Md Liakat Ali and Caio Canella Vieira from the Fisher Delta Research Center for their generous supports of research fields, devices and data collection, Drs. Henry T. Nguyen and Heng Ye from the Molecular Genetics and Soybean Genomics Laboratory for their patient guidance in soybean genetics and supports of plant materials, and Dr. Andrew Scaboo from the Missouri Soybean Association for granting me access to his experimental materials and data. I am very grateful for having the privilege to closely work with these great scientists and learn perspectives, ideas, and knowledge from them.

I would thank the current and past members of PAAL, Dr. Xiuqing Fu, Dr. Shuiqin Zhou, Wenyi Cao, Dr. Dandan Fu, Huawei Mou and Aijing Feng for their kind help in preparing for experiments and collecting data. A special thanks to my co-worker and life- 
long friend Chin Nee Vong for her endless help in every moment that I have needed.

I am also grateful to the other two members of my doctoral committee, Drs. Gang Yao and Leon Schumacher for taking time out of their busy schedules to discuss my research progress and provide valuable suggestions towards completing the doctoral program. I have learned many skills in scientific writing and presenting by attending Dr. Yao's classes and admire his serious attitude and organized ways in preparing class materials. It was also a precious experience to be a teaching assistant in Dr. Schumacher's class. He is one of the most interesting persons that I have ever met. I was impressed by his optimism and the considerations he has put on each of his students.

Last but not least, I am very appreciative of my parents for their unconditional love and support wherever I am in the world. I would also thank my partner Xiaotian Hu for her love, encourage and willingness to sharing every moment of my life whether sad or joyful. A special thanks to my cat, Burger for joining my family and meowing at me. I want to give my appreciation to every friend who I met during this special period of my lifetime.

Things that I have obtained from completing the dissertation are much more than earning a Ph.D. degree. They are the treasure of my life and will benefit me for the rest. 


\section{TABLE OF CONTENTS}

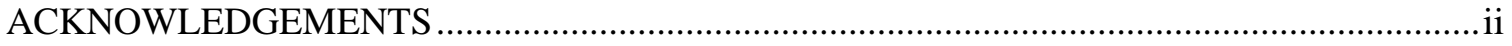

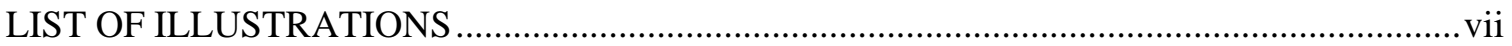

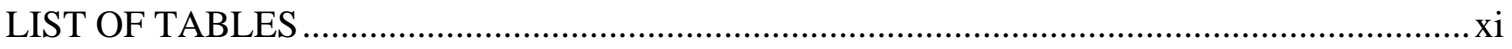

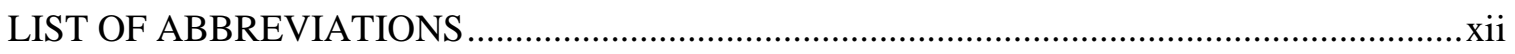

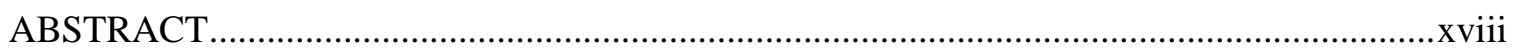

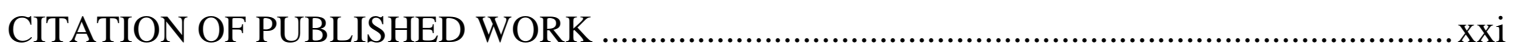

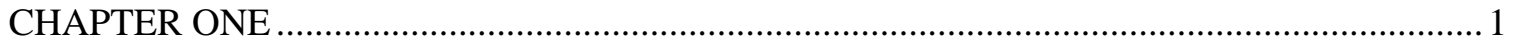

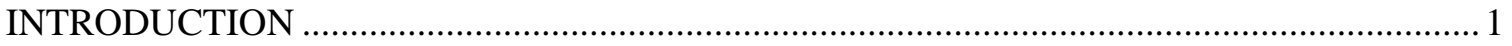

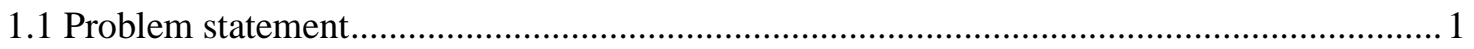

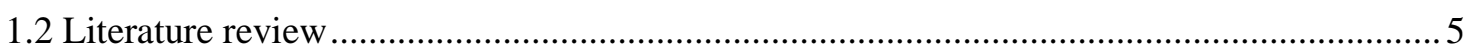

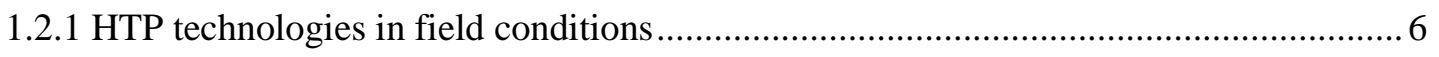

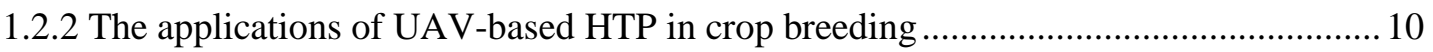

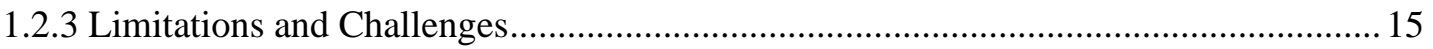

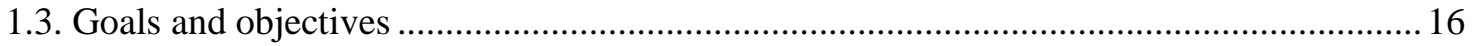

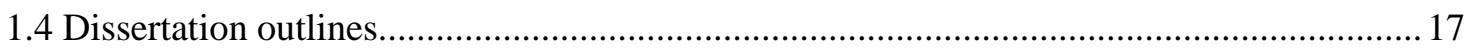

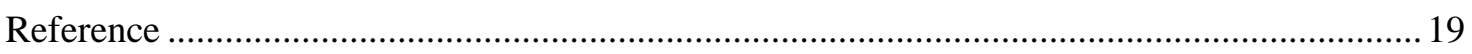

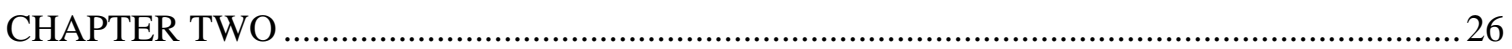

ESTIMATION OF THE MATURITY DATE OF SOYBEAN BREEDING LINES USING UAV-

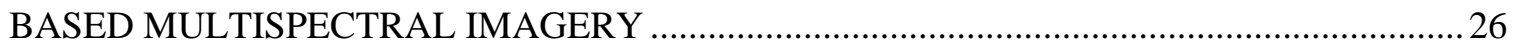

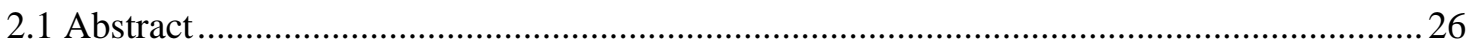

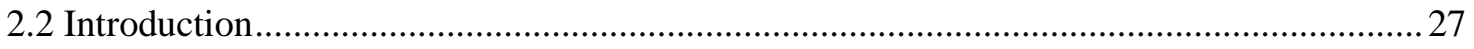

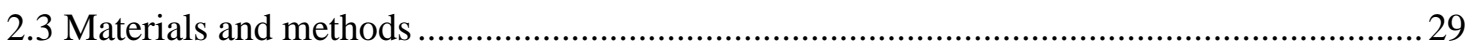

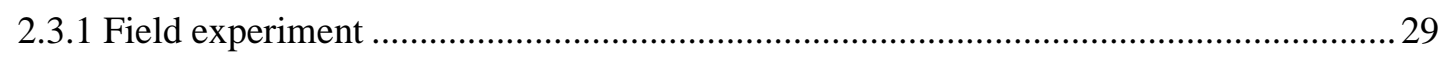

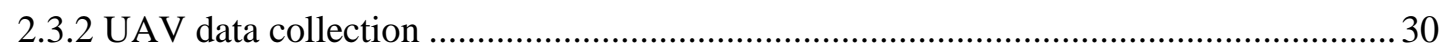

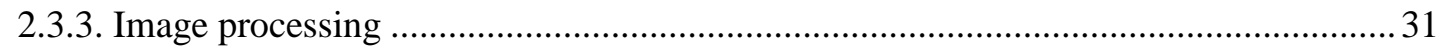

2.3.4. Maturity date and adjusted maturity date..................................................................... 34

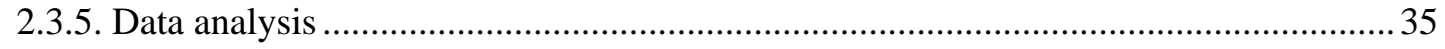

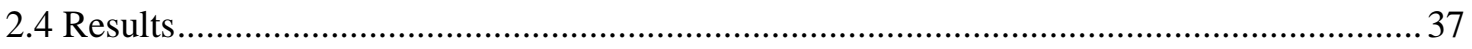

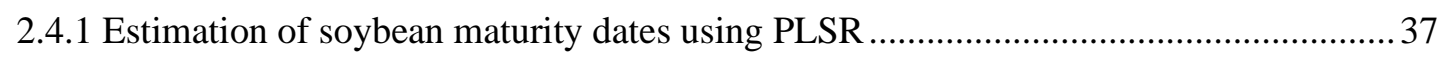

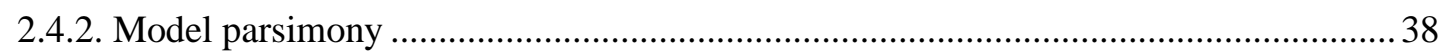

2.4.3. Adjusted maturity dates based on the variances in image features ............................... 41

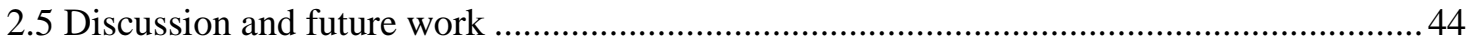

2.5.1. Estimation of soybean maturity dates at different growth stages................................. 44

2.5.2. Selected features for the parsimonious models ........................................................... 45 


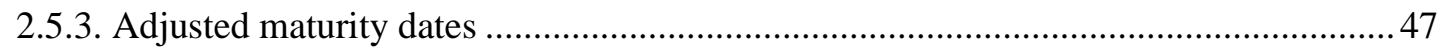

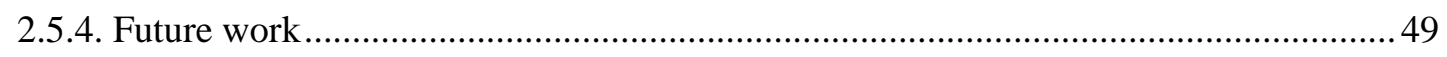

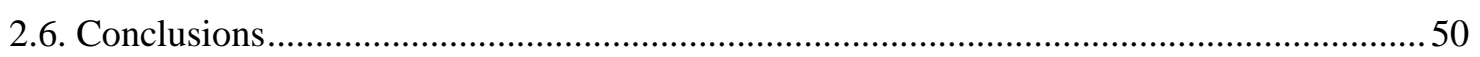

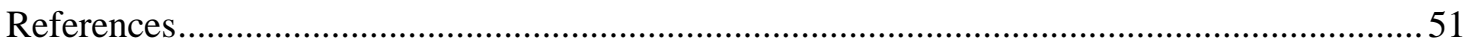

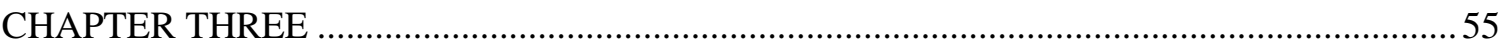

YIELD ESTIMATION OF SOYBEAN BREEDING LINES UNDER DROUGHT STRESS USING UNMANNED AERIAL VEHICLE-BASED IMAGERY AND CONVOLUTIONAL

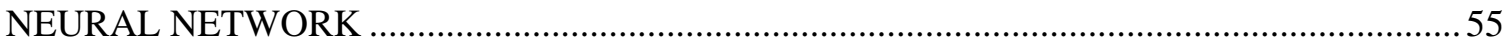

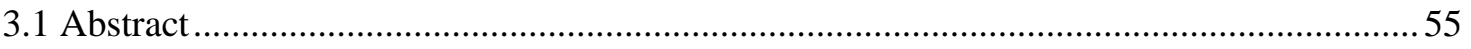

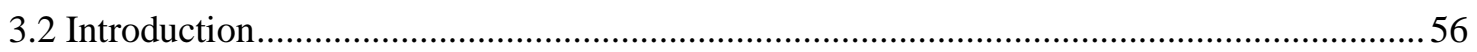

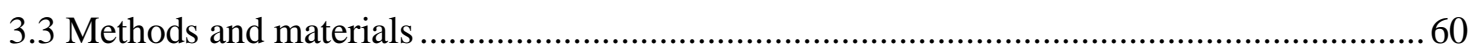

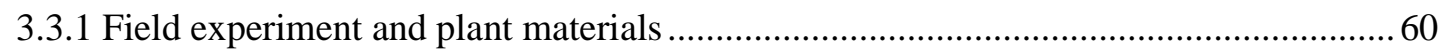

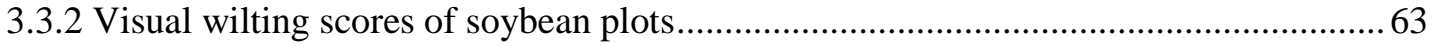

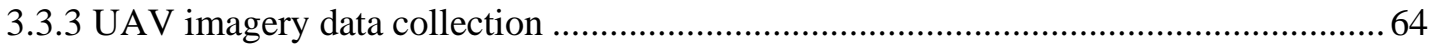

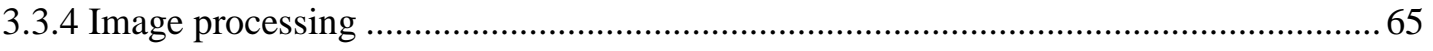

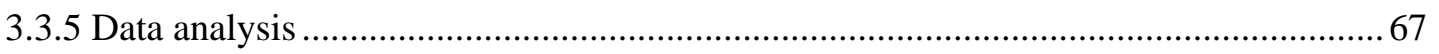

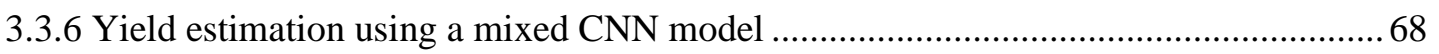

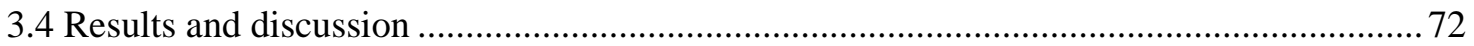

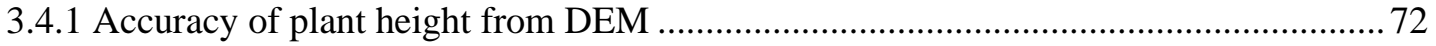

3.4.2 The effects of the maturity and wilting trait on yield..................................................... 73

3.4.3 Image features collected at three growth stages............................................................ 75

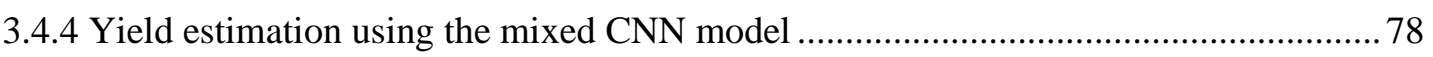

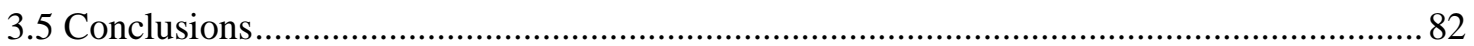

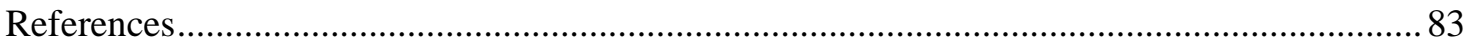

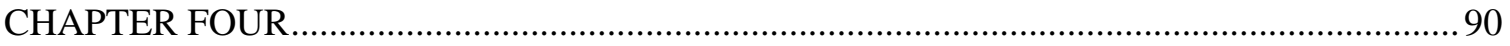

VARIETY SELECTION USING UAV HIGH-THROUGHPUT PHENOTYPING TOWARDS IMPROVE SELECTION ACCURACY AND INTENSITY FOR SOYBEAN BREEDING ......90

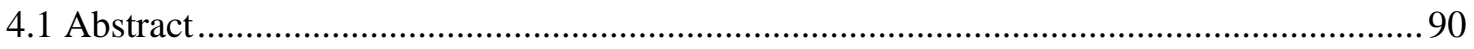

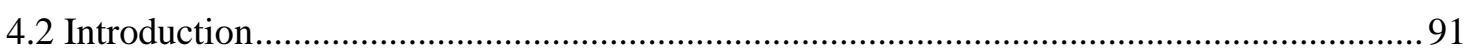

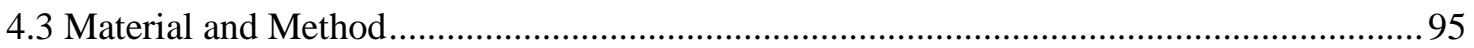

4.3.1 Plant material and field experiment ......................................................................... 95

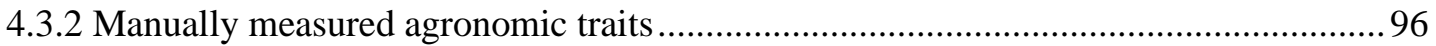

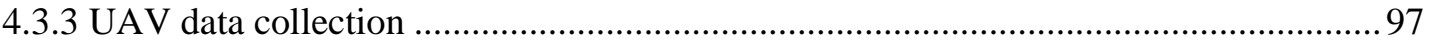

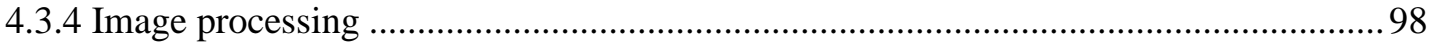

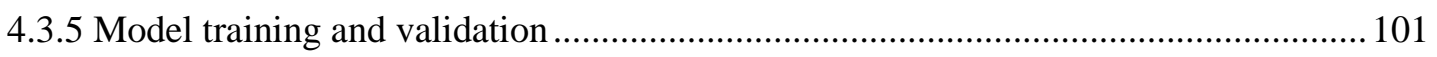

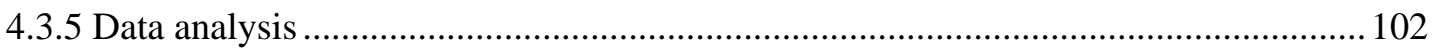

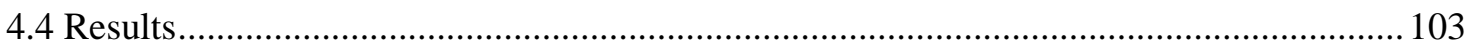


4.4.1 Differences in the manually measured agronomic traits between the selected and unselected groups

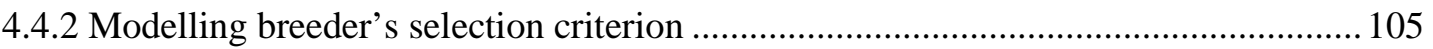

4.4.3 Differences in image features between selected and non-selected varieties ................ 108

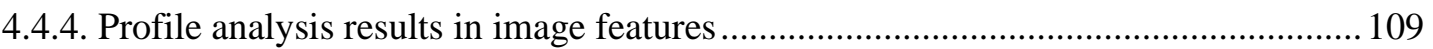

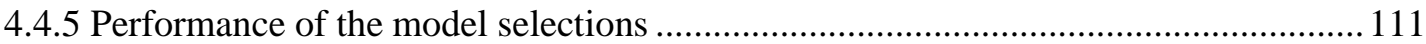

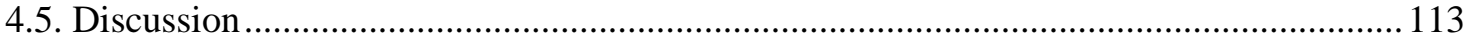

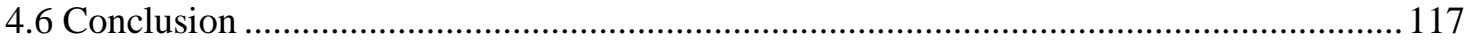

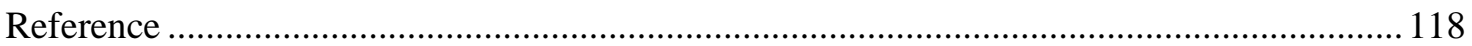

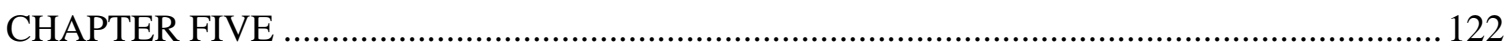

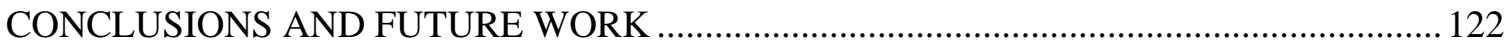

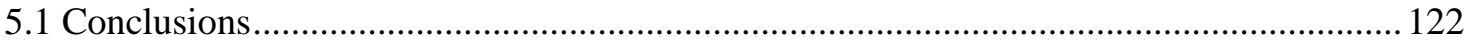

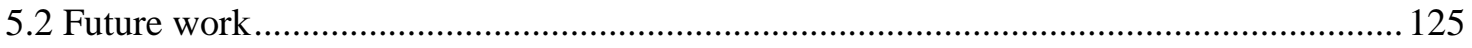

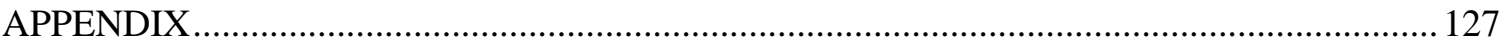

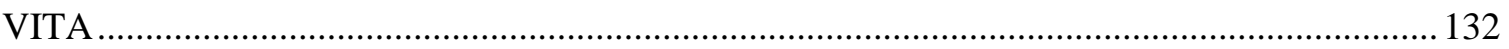




\section{LIST OF ILLUSTRATIONS}

Figure 1-1. Soybean breeding timeline

Figure 1-2. Electromagnetic spectrum (EMS) and sensors corresponding to different regions of wavelength (Fahlgren et al., 2015). 7

Figure 2-1. Orthomosaic images of part of the soybean field. (a) and (c) are the colorful images with the red (r), green (g) and blue (b) channels of images acquired on Aug. 27 and Sept. 27. (b) The grayscale image of blue normalized difference vegetation index (BNDVI) derived from images taken on Aug. 27 and (d) the grayscale image of blue-wide dynamic range vegetation index (BWDRVI) derived from images taken on Sept. 27. (e) and (f) are histograms of image pixel values in the two red rectangles.

Figure 2-2. Illustration of variations in canopy images of the soybean lines matured on Sept. 25. Images were generated using $\mathrm{r}$, $\mathrm{g}$ and $\mathrm{b}$ bands from the multispectral camera. Breeding line no. from left to right is: 7344, 7335 and 6913.

Figure 2-3. Estimation of maturity dates using partial least square regression (PLSR). (a) Percent of variance explained in the maturity dates as a function of the number of components in the PLSR model. (b) Mean square error in the maturity dates as a function of the number of components. (c)-(e) The correlations between manual maturity dates and predicted maturity dates using images features taken on Aug. 27, Sept. 14 and Sept. 27, respectively. ncomp represents the optimal number of components.

Figure 2-4. Variable importance in projection (VIP) scores of 130 predictors of the PLSR models on the three days.

Figure 2-5. Estimation of maturity dates using PLSR models with selected images features and the changing rates. (a) The correlations between manual maturity dates and predicted maturity dates using the selected images features taken on 14 September and their changing rates between 27 August and 14 September. (b) The correlations using the selected images features taken on 14 September and their changing rates between 14 September and 27 September. (c) The correlations using the selected images features taken on 27 September and their changing rates between 14 September and 27 September.

Figure 2-6. Vegetation indices CCCI and NDVIrededge. (a)-(b) The relationships between maturity dates and NDVIrededge_mean and CCCI_mean collected on Sept. 14 and 27. (c) Canopy images of the soybean lines matured on Sept. 25 in RGB, CCCI and NDVIrededge...... 43 
Figure 2-7. Adjusted maturity dates. (a) The relationship between maturity dates and the adjusted maturity dates based on the variances of NDVIrededge_mean and CCCI_mean taken on Sept. 14 and 27. (b) The correlations between adjusted maturity dates and predicted maturity dates using the selected image features taken on Sept. 14 and their changing rates between Aug. 27 and Sept. 14 (c) The correlations using the selected image features taken on Sept. 14 and their changing rates between Sept. 14 and 27. (d) The correlations using the selected image features taken on Sept. 27 and their changing rates between Sept. 14 and 27.

Figure 2-8. The changing rates in CI_mean and hue_std from Sept. 14 and 27. (a) The changing rates in CI_mean and hue_std. (b) and (d) are RGB images of a line matured on Sept. 19, and they were taken on Sept. 14 and 27, respectively. (c) and (e) are RGB images of a line matured on Oct. 1, and they were taken on Sept.14 and 27, respectively 47

Figure 3-1. Publications about crop yield using UAV imagery. .58

Figure 3-2. The field experiment. (a) The test site location. (b) Daily average temperature and cumulative precipitation during the growing season (c) Illustration of the experimental blocks in the field.

Figure 3-3. The architecture of the mixed CNN model.

Figure 3-4. Comparison of the height from DEMs with manual measurements. (a) The deviations of the GCPs height from DEM. (b) Agreement of the height from DEMs and manual measurement of nine soybean plots collected on July 7 and Aug. 22. Black solid line: a reference line $(y=x)$. Orange dash line: a least square linear fit of the orange dots $(y=1.8 x-28.5)$. Blue dot line: a least square linear fit of the blue dots $(y=1.1 x-9.1)$.

Figure 3-5. The bar plots of the effects of maturity group, wilting trait, and their interactions on yield. (a) is the yield means of the fast- and slow-wilting groups. (b) is the yield means of three MGs. (c) is the yield means of three MGs with different wilting traits. The lower-case letters above bars indicate the significant difference among these means at a 0.001 significance level.. 74

Figure 3-6. Image features collected at three growth stages. (a) - (f) are boxplots of six image features, i.e. hue, saturation, ExG, height, NDVI, and gNDVI collected at three growth stages. The lower-case letters indicate the significant difference among these means at a 0.05 significance level. (g) Pearson correlations between yield and image features collected at three dates. NS indicates No Significance. ${ }^{* *}$, ${ }^{* * *}$ indicate the significance levels of $p=0.01$ and 0.001 , respectively. 
Figure 3-7. The performance of yield estimated by the CNN branch and the mixed CNN model with seven image features as predictors. (a) and (c) are the scatter plots of losses against epochs. (b) and (d) are the scatter plots of the predicted vs. the measured yield. Blue dot lines: least square linear fits of the dots ( $y=1.4 x$-393 and $y=1.5 x$-399 for the lines in (b) and (d), respectively). (a) and (b) are for the mixed CNN model. (c) and (d) are for the CNN model.

Figure 3-8. Images of soybean lines with yield in five levels. (a) - (e) are images in RGB, MTVI2, DEM, CVI_contrast, and CVI_entropy, respectively.

Figure 4-1. Illustration of the experimental fields. (a) The progeny trial in 2018. (b) The preliminary yield trial in 2019. (c) The advanced yield trial in 2020. (d) Locations of the fields. 96

Figure 4-2. The image processing pipeline

Figure 4-3. Illustration of two metrics for evaluating model performance for selecting superior soybean plots.

Figure 4-4. Comparisons of manually measured agronomic traits between the selected and nonselected groups in the PYT. (a) - (d) Boxplots of the comparisons in yield, height, lodging and moisture, respectively. The lower-case letters above bars indicate the significant difference among these means at the above significance levels (p-values). (e) Histogram of the maturity dates. (f) and (g) Bar plots of the flower and pubescence colors. For (e)-(g), the blue bars represent all plots, while the orange bars represent the selected plots.

Figure 4-5. Comparisons of manually measured agronomic traits between the selected and nonselected groups in the AYT. (a) - (d) Boxplots of the comparisons in yield, height, lodging and moisture, respectively. The lower-case letters above bars indicate the significant difference among these means at the above significance levels (p-values). (e) Histogram of the maturity dates. (f) and (g) Bar plots of the flower and pubescence colors. For (e)-(g), the blue bars represent all plots, while the orange bars represent the selected plots.

Figure 4-6. Manually measured agronomic traits in PYT. (a) are the visualization plots of the variables' contributions and directions in the plane of the first two PCs. (b) is the projection of sample variables on the first two PCs. (c)-(d) are scatterplots of yield versus height, lodging and maturity date, respectively. (h) is a scatterplot of the relationship between moisture and maturity date. Eight outliers with moisture values higher than $20 \%$ were removed to ensure the adequacy of linear regression. (g)-(h) are boxplots of the yield of three groups of flower color and three groups of pubescence color. The p-value above (c)-(h) indicates the significance level of estimation. 107 
Figure 4-7. Manually measured agronomic traits in PYT. (a) are the visualization plots of the variables' contributions and directions in the plane of the first two PCs. (b) is the projection of sample variables on the first two PCs. (c)-(e) are scatterplots of yield versus height, lodging and maturity date, respectively. (f) is a scatterplot of the relationship between moisture and maturity date. (g)-(h) are boxplots of the yield of three groups of flower color and three groups of pubescence color. The $p$-value above (c)-(h) indicates the significance level of estimation........ 108

Figure 4-8. Illustration of the differences in image features between the selected and non-selected groups. Each square represents if there is a significant difference in an image feature collected at single times ( $\mathrm{p}$-value $<0.05$ ) between the two groups and which had higher values. 109

Figure 4-9. Profile plots of GNDVI for the selected and non-selected groups over the growing seasons. (a) - (c) are plots for the PT, PYT and AYT, respectively. The lower-case letters above bars indicate the significant difference among these means at a 0.05 significance level. The pvalue above each plot indicates the significance level of the null hypothesis that the two groups had a parallel pattern over time in GNDVI. Appendix Figure A1 shows the profile plots with different GNDVI scales.

Figure 4-10. Model performance on selecting superior soybean lines from the PT and PYT. (a) and (c) Comparison of the PYT yield means. (b) and (d) Comparison of the AYT yield means. (a)-(b) are for the PT selections, while (c) and (d) are for the PYT selections. Orange and blue bars represent the model selections (the TP class) and model non-selections but selected by breeders (the FN class), respectively. Error bars show the standard deviations. *, **, *** indicate the significance levels of $p=0.05,0.01$, and 0.001 for testing the hypothesis that there is no difference in yield means between model selections and non-selections. NS represents no significance. The numbers in blue are the classification recall 


\section{LIST OF TABLES}

Table 2-1. Wavelength information of the camera and the reflectance in each band of the calibration reflectance panel (CRP).

Table 2-2. Pearson correlations between the predictors with VIP scores $\geq 1$ and variance inflation factors (VIFs) $<5$ and maturity dates on the three days.

Table 2-3. Pearson correlations between maturity dates and the means of image features at each maturity dates on the three days

Table 3-1. The number of soybean breeding lines in the six groups (3 MGs $\times 2$ wilting traits)... 63

Table 3-2. Descriptions of three vegetation indices and texture features used in the mixed CNN model. The variables in the formulas of VIs are the pixel values of different channels of the 5band multispectral. $\mathrm{p}_{\mathrm{ij}}$ is the pixel value of the $\mathrm{i}^{\text {th }}$ row and the $\mathrm{j}^{\text {th }}$ column in an image. $\mathrm{N}$ is the dimension of the moving window (kernel) when calculating the grey level co-occurrence matrix of each image for texture features 


\section{LIST OF ABBREVIATIONS}

$1 \mathrm{D}$

2D

adMD

AEN

AGL

ANN

ANOVA Analysis of Variance

API

AYT

B

BNDVI Blue Normalized Difference Vegetation Index

BWDRVI Blue-Wide Dynamic Range Vegetation Index

CCCI Canopy Chlorophyll Content Index

CDT

CI

CNN Convolutional Neural Network

CRP Calibration Reflectance Panel

CVI Chlorophyll VI

DAP Day After Planting 


\begin{tabular}{|c|c|}
\hline DEM & Digital Elevation Model \\
\hline DL & Deep Learning \\
\hline $\mathrm{DN}$ & Digital Number \\
\hline DNN & Deep Neural Network \\
\hline EMS & Electromagnetic Spectrum \\
\hline ExG & Excess Green \\
\hline EXIF & Exchangeable Image File Format \\
\hline fAPAR & Fraction of Absorbed Photosynthetically Active Radiation \\
\hline FN & False Negative \\
\hline fps & Frame Per Second \\
\hline fps & Frame Per Second \\
\hline FWHM & Full Width at Half Maximum \\
\hline G & Green \\
\hline GCP & Ground Control Point \\
\hline GDVI & Green Difference VI \\
\hline GEBVs & Genomic-Estimated Breeding Values \\
\hline GLI & Green leaf index \\
\hline gNDVI & Green NDVI \\
\hline GNSS & Global Navigation Satellite System \\
\hline GRNDVI & Green-Red NDVI \\
\hline
\end{tabular}




\begin{tabular}{|c|c|}
\hline GRVI & Green Ratio VI \\
\hline GS & Genomic Selection \\
\hline GSD & Ground Sampling Distance \\
\hline $\mathrm{G}_{\mathrm{y}}$ & Genetic Gain \\
\hline h & Selection Accuracy \\
\hline $\mathrm{H}$ & Hue \\
\hline $\mathrm{H}^{2}$ & Broad-sense Heritability \\
\hline HSD & Honest Significant Difference \\
\hline HSV & Hue-Saturation-Value Color Space \\
\hline HTP & High-Throughput Phenotyping \\
\hline IF & Shape Index \\
\hline IO & Iron Oxide \\
\hline $\mathrm{k}$ & Selection Intensity \\
\hline Lasso & Least Absolute Shrinkage and Selection Operator \\
\hline LiDAR & Light Detection and Ranging \\
\hline MANOVA & Multivariate ANOVA \\
\hline MAS & Marker Assisted Selection \\
\hline MAV & Manned Aerial Vehicle \\
\hline MG & Maturity Group \\
\hline ML & Machine Learning \\
\hline
\end{tabular}




\begin{tabular}{|c|c|}
\hline MTVI2 & Modified Triangular Vegetation Index \\
\hline MU & University of Missouri \\
\hline $\mathrm{N}$ & Dimension of the moving window for the grey level co-occurrence matrix \\
\hline NDVI & Normalized Difference Vegetation Index \\
\hline NDVIrededge & Normalized Difference Rededge/Red \\
\hline NIL & Near Isogenic Line \\
\hline NIR & Near-Infrared \\
\hline NormG & Norm Green \\
\hline NS & No Significance \\
\hline PCA & Principal Component Analysis \\
\hline PLSR & Partial Least Square Regression \\
\hline PT & Progeny Trial \\
\hline PYT & Preliminary Yield Trial \\
\hline QTL & Quantitative Trait Locus \\
\hline $\mathrm{R}$ & Red \\
\hline$r$ & Pearson Correlation \\
\hline $\mathrm{R}^{2}$ & Correlation of Determination \\
\hline R2 & Reproductive growth Stage 2 \\
\hline R3 & Reproductive growth Stage 3 \\
\hline R5 & Reproductive growth Stage 5 \\
\hline
\end{tabular}


RaDAR Radio Detection and Ranging

Re $\quad$ Red Edge

ReLU Rectified Linear Unit

RGB Red-Green-Blue

RGR Red-Green Ratio

RIL Recombinant Inbred Line

RM Relative Maturity

RMSE Root Mean Square Error

RNN Recurrent Neural Network

S Saturation

SfM Structure from Motion

std Standard Deviation

TBVs True Breeding Values

TGI Triangular greenness index

TP True Positive

UAV Unmanned Aerial Vehicle

V Value

V4 Vegetative growth Stage 4 
VI

VIF

VIP

y

YT

$\sigma_{\mathrm{A}}$
Vegetation Index

Variance Inflation Factor

Variable Importance in Projection

Number of years required to complete a breeding cycle

Yield Trial

Genetic Variance 


\title{
DEVELOPMENT OF UAV-BASED HIGH-THROUGHPUT PHENOTYPING
}

\section{TECHNOLOGIES TOWARDS IMPROVING SOYBEAN BREEDING EFFICIENCY}

\author{
Jing Zhou
}

Dr. Jianfeng Zhou, Dissertation Supervisor

\begin{abstract}
Development of new crop varieties with improved traits through crop breeding programs is one of the promising solutions for the estimated food crisis in 2050 when agricultural production needs to double its current growth rate to feed 10 billion people in the world. Conventional crop breeding strategies largely rely on 'trial-and-error' and human input, limiting the trial capacity of breeding materials, the accuracy of measuring plant traits, and selection intensity of elite genotypes. These limitations have become a bottleneck to boost breeding efficiency as it is expected to meet the food demands.

The goal of this research was to develop an integrated and automated highthroughput phenotyping (HTP) framework leveraging advanced technologies in remote sensing and artificial intelligence for estimating key traits and selecting elite genotypes towards improving the selection intensity and accuracy of conventional soybean breeding. To achieve this goal, this research features three objectives: (1) develop an integrated and automated UAV HTP framework for measuring crop traits accurately and efficiently, (2) estimate soybean yield and maturity date of breeding materials using UAV image features and machine learning models., and (3) select elite soybean lines using UAV image features towards improving the selection intensity and accuracy.
\end{abstract}

A UAV-based HTP platform was developed to carry multispectral and high- 
resolution digital cameras and geo-referencing units. The platform was able to cover a 9acre field within 2 hours. An automated pipeline was developed to process the collected time-series images and generate labeled image features. It was shown that the developed methods can deliver accurate measurements on plant height (coefficient of determination $R^{2}$ up to 0.90 with average errors within $5 \mathrm{~cm}$ ) and consistent spectral reflectance.

The UAV platform and image processing pipeline were applied to estimate two key agronomic traits for soybean breeding, i.e., the maturity date and yield. A group of image features was collected on 326 soybean progeny lines near their maturity stages (R7-8). Their maturity dates were estimated using a partial least square regression (PLSR) model with the image features as inputs and the visual maturity dates taken by breeders as outputs. The results showed that the image-based maturity dates highly agreed $\left(R^{2}=0.81\right)$ with the visual ones with the root mean square error (RMSE) of 1.4 days.

For estimating the soybean yield, 972 soybean breeding plots in three maturity groups were planted under rainfed conditions. A mixed convolutional neural network (CNN) model was built to estimate soybean yield by taking seven image features (associated with plant height, canopy color, and canopy texture) and two categorical factors, i.e. maturity group and drought tolerance, as predictors. The prediction model could explain $78 \%$ of the measured yield with an RMSE of $391.0 \mathrm{~kg} \cdot \mathrm{ha}^{-1}$ (33.8 \% to average yield).

To model the breeder's selection criteria and select elite soybean genotypes, a soybean breeding program was traced for three years. The progeny trial (PT) had 11,473 rows, and 1,773 among them were selected for a preliminary yield trial (PYT) and 238 were further selected for an advance yield trial (AYT). Seven agronomic traits, including 
yield, plant height, maturity data, flower and pubescence color, moisture and lodging were manually measured for soybeans in the two yield trials. The UAV imagery was collected every two weeks over the growing seasons, and a group of image features was extracted for each trial. Results show the progeny lines had the most variation among the three trials and the images collected at earlier stages (before R5) explained more variation than those at later stages. A Lasso model for selecting soybean lines with image features correctly identified $71 \%$ and $76 \%$ of the breeder's selection for the PT and PYT. The model selections in PT and PYT had respectively $4 \%$ and 5\% higher yield, comparing the breeder's selection.

In summary, the developed UAV HTP platform is capable of collecting image features of soybean breeding materials efficiently and delivering estimations of agronomic traits accurately. The accurate and subjective estimations of plant traits decrease the phenotypic variations in breeding trials. By liberating human labor from the onerous field evaluation, the population size of soybean breeding lines could be increased a lot, leading to increased selection intensity. Moreover, the proposed variety selection model was able to narrow down the breeder selections, further increasing the selection accuracy and intensity. Therefore, it is be concluded that the developed UAV HTP platform has great potential in improving soybean breeding efficiency by decreasing the phenotypic variations, increasing the selection accuracy and intensity. This research could be scaled up to other crop breeding programs and offered a paradigm of improving the breeding efficiency using HTP technologies. 


\section{CITATION OF PUBLISHED WORK}

Two chapters of this dissertation have been published as follows:

\section{Chapter 2}

Zhou, J., Yungbluth, D., Vong, C. N., Scaboo, A., \& Zhou, J. (2019). Estimation of the maturity date of soybean breeding lines using UAV-based multispectral imagery. Remote Sensing, 11(18), 2075. https://doi.org/10.3390/rs11182075

\section{Chapter 3}

Zhou, J., Zhou, J., Ye, H., Ali, M. L., Chen, P., \& Nguyen, H. T. (2021). Yield estimation of soybean breeding lines under drought stress using unmanned aerial vehiclebased imagery and convolutional neural network. Biosystems Engineering, 204, 90-103. https://doi.org/10.1016/j.biosystemseng.2021.01.017 


\section{CHAPTER ONE \\ INTRODUCTION}

\subsection{Problem statement}

Phenotyping is essential to plant breeding (Fasoula et al., 2020). From 10,000 years ago, plants with preferrable phenotypes have been selected and bred by human beings for higher productions and environmental adaptivity (Ahmar et al., 2020). Since the early 1900s, crop development has been systemized with the introduction of Mendelian laws, followed by cross-breeding and hybrid breeding, and was further revolutionized with the availability of high-throughput genome information. However, it requires the accurate quantification of plant traits (phenotyping) to evaluate plant performance, characterize germplasm and experimental populations to identify useful genes/QTLs (Mir et al., 2019), or to train a prediction model, no matter in conventional breeding or molecular breeding (Lorenz et al., 2011). Similarly, phenotyping is also required to evaluate the results of mutagenesis, genetically modified organisms (Blum, 2014), or even clustered regularly interspaced short palindromic repeats (CRISPR)/CRISPR-associated protein-9 nuclease (CRISPR/Cas9) (Araus et al., 2018).

A fundamental concept in breeding is genetic gain $\left(G_{y}\right)$ that is the amount of increase in performance of a population achieved in one year through artificial selection (Fehr, 1991) as defined in Eq.1-1.

$$
G_{y}=\frac{k h \sigma_{A}}{y}
$$

Where $k$ is selection intensity, $h$ is selection accuracy, $\sigma_{A}$ is genetic variance and $y$ is the number of years required to complete a breeding cycle. In the context of phenotypic 
(conventional) selection, $h$ equals the square root of the narrow-sense heritability, while in the context of genomic selection (GS), $h$ is the correlation between true breeding values (TBVs) and genomic-estimated breeding values (GEBVs) (Xu et al., 2017). The equation of genetic gain translates the efficiency of selection in plant breeding programs into measurable parameters that breeders could manipulate in their pipelines. In other words, the genetic gain can be increased by increasing population size (or selecting fewer numbers of varieties) to increase selection intensity, shortening the breeding cycle, ensuring suitable genetic variation in the population, and obtaining accurate estimates of the genetic and phenotypic values (Araus et al., 2018; Moreira et al., 2019; Xu et al., 2017).

Crop breeding is the art and science of changing the traits of crops to produce desired characteristics (Sleper \& Poehlman, 2006). Specifically, the changes of traits for a crop type are made by identifying and selecting varieties with desired traits in its given population and combining these into one individual plant (BATS Center, 1995). The desired crop traits include improved yield primarily and other aspects of agronomic importance, for example, biotic and abiotic tolerance, appropriateness (e.g., crop height) for agricultural management and operations.
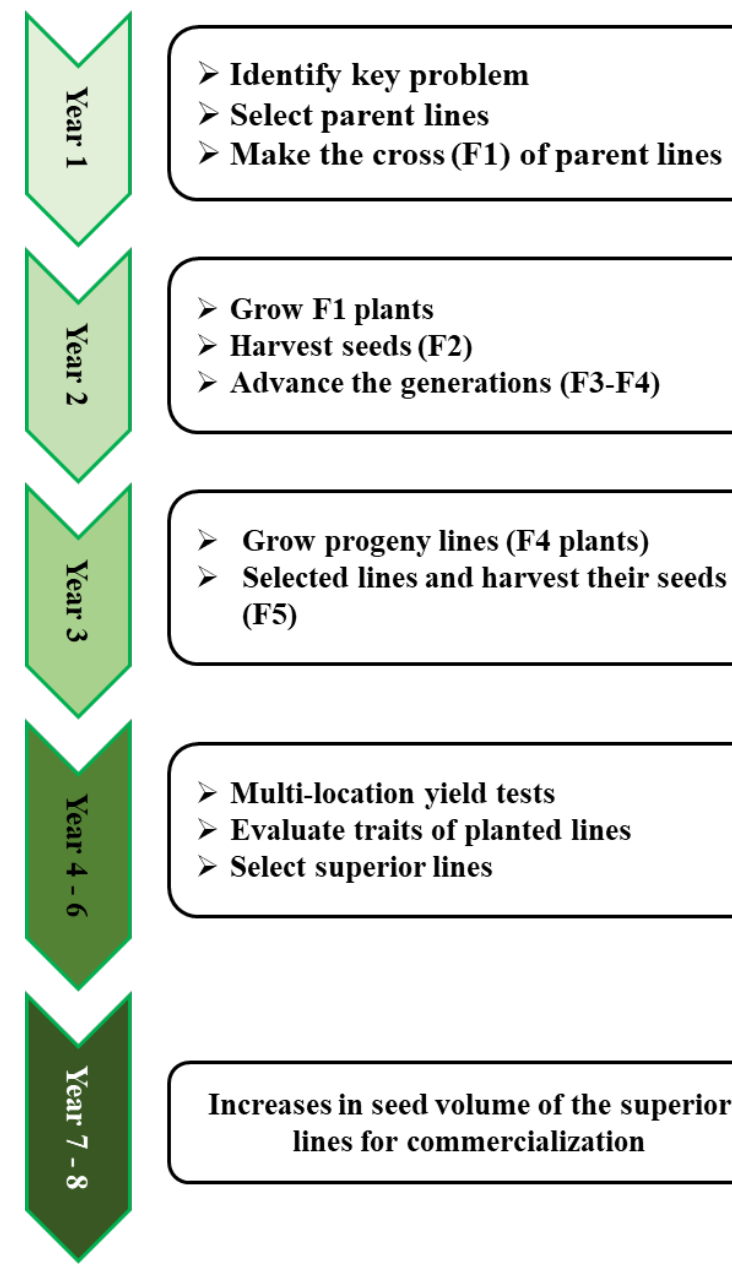

$>$ Advance the generations (F3-F4)

$>$ Grow progeny lines (F4 plants)

$>$ Selected lines and harvest their seeds (F5)

$>$ Multi-location yield tests

Evaluate traits of planted lines

Select superior lines

Increases in seed volume of the superior lines for commercialization

Figure 1-1. Soybean breeding timeline 
In conventional breeding, given many phases of crossing, selection, and testing involved in the production of new plant varieties, it can take one or two decades to create a new cultivar (Ahmar et al., 2020). There are several procedures following the timeline (as shown in Figure 1-1) to combine desired crop traits into individual crops and select superior varieties eventually. Taking a soybean breeding program as an example, roughly it takes seven to eight years to develop new soybean varieties in conventional crop breeding programs (Krueger, 2019). After identifying a key problem in a specific program, breeders select from parent lines from given populations that have desired traits to potentially address the problem. Then the selected parent lines are combined to obtain crosses that probably would exhibit desired traits from both parents simultaneously. The crosses are planted and harvested individually, and their generations are advanced to produce enough seeds for planting progeny lines. Then the progeny lines are selected for a combination of the desired traits eliminating those inheriting undesired traits from parents. The selected progeny lines are kept for multi-location yield trials in which breeders could keep selecting lines that are adapted either widely across the regions or specifically for targeted regions, depending on the purpose of the program.

The conventional breeding cycle can be shortened by accelerating breeding procedures through integrated breeding approaches assisted with genetic markers (Xu et al., 2017), such as marker assisted selection (MAS) and GS. Genetic markers are the biological features that are determined by allelic forms of genes or genetic loci and can be transmitted from one generation to another. Thus they can be used as experimental probes or tags to keep track of an individual, a tissue, a cell, a nucleus, a chromosome or a gene (Jiang, 2013). MAS refers to a breeding procedure using genetic markers associated with 
desirable traits as a substitute for or to assist conventional phenotypic screening (Fu et al., 2017). GS aims to use genome-wide markers to estimate the effects of all loci and thereby predict the genetic values of untested populations to achieve a comprehensive and reliable selection for accelerating genetic progress in crop breeding (Dwivedi et al., 2020). MAS and GS rely on marker-trait associations and they both have a prerequisite to use the markers in such selections that they should be closely linked to the precise phenotype or quantitative trait loci (QTLs) of interest and polymorphism for the markers between the parents (Shu \& Wu, 2016).

Despite genomics and technical advances, identifying plants with desirable traits is the initial and most important step in breeding pipelines (Ahmar et al., 2020). It is well acknowledged in the breeding community that phenotyping efficiency still represents a bottleneck in crop breeding (Araus \& Cairns, 2014; Araus et al., 2018; Furbank \& Tester, 2011), as phenotypes are the bases to make selections in conventional breeding and a lack of suitable phenotyping data has led to poor results in gene/QTL discovery, limiting progress in genomics-assisted crop improvement programs (Mammadov et al., 2012).

Over the years, an explosion in state-of-the-art technologies, developed through collaborative efforts of multidisciplinary teams, to assist the process of high-throughput phenotyping (HTP) in crop breeding (Fasoula et al., 2020). In terms of improving genetic gain, HTP contributes directly to (1) increase selection accuracy by increasing the accuracy of phenotypic information; (2) increase selection intensity by increasing population size (or reducing the number of selected plants) with the increased phenotyping efficiency and (3) identify novel genetic variations. Extensive adaptations of HTP in breeding pipelines will indirectly impact (1) breeding circles with increase selection accuracy and intensity in 
yearly trials, (2) budget allocation with reduced human input, (3) development of genetic selection with the availability of accurate phenotypic data in training populations.

Although the HTP advances have been proven capable of measuring a wide range of plant phenotypic traits, benefited by the rapid development of sensors and hardware platforms, the wide adoption of HTP in breeding routine procedures is hindered by a few limitations and challenges (Araus et al., 2018). There is an urgent need to ensure that these advances find practical application in breeding programs and contribute toward increased genetic gain.

\subsection{Literature review}

A phenotype is the result of interactions between plant genome and micro-and mega-environments encountered during its life span (Fasoula et al., 2020). Phenotyping is referred to as the set of methodologies (particularly noninvasive technologies) and protocols used to measure plant growth, architecture, and composition with certain accuracy and precision at different scales of organization, from organs to canopies (Fiorani \& Schurr, 2013). Although there is not an official definition for the HTP, it is generally considered as an integrated system that can collect massive amounts of phenotypic data from hundreds (and more) of plants on a regular basis (days to months) with a high degree of automation (D. Li et al., 2021; Yang et al., 2017). With this broad definition, a diversified range of HTP systems has been developed at different scales of organization (from organs to canopies) of different types of crops under various conditions (from lab to field) for diverse purposes. The following contents summarize current HTP technologies applied in field conditions, HTP applications on crop breeding (conventional and molecular) as well as the limitations and challenges to be addressed before meeting the 
needs for future breeding advances.

\subsubsection{HTP technologies in field conditions}

\section{(1) Field HTP platforms}

HTP can be implemented in field conditions with a variety of forms, mainly categorized into ground-based and aerial platforms. Ground-based platforms mainly include pole/tower-based systems, gantry-based systems, ground mobile systems, and cable-suspended systems. Ground-based platforms can provide data with higher spatial and temporal resolutions than the aerial ones, have the flexibilities to be equipped with multiple devices and usually do not require special operation skills, such as a remote pilot license. However, they have relatively low efficiency in terms of field coverage (D. Li et al., 2021).

Based on imaging distance, there are three types of aerial HTP platforms, namely unmanned aerial vehicle (UAV), manned aerial vehicle (MAV), and satellite platforms. As imaging distances increasing, the aerial platforms have decreased image pixel resolutions, but accordingly with increased ability to cover large-scale fields (from a few to hundreds of acres) within a short time period. The UAVs and MAVs require operations from at least two dedicated persons and devices to provide information from the global navigation satellite system (GNSS) together with ground control points (GCPs). With limited controls on environmental lights, calibration procedures are necessary to correct light variations in images collected by the aerial platforms. Satellite platforms can provide imagery in several spectral bands at meter-level resolutions as well as radio detection and ranging (RaDAR) data (Jin et al., 2021). Processing satellite images is at a relatively low cost due to the international standard protocols and the accessibility of commercial satellite platforms, such as the Landsat series and WorldView series (C. Zhang et al., 2020). Satellite imagery 
is susceptible to weather conditions, such as cloud, rain, fog and haze (D. Li et al., 2021), which is gradually compensated by the application of RaDAR data on sensing crop traits.

\section{(2) Sensors}

Sensors that are commonly equipped in the field HTP platforms including redgreen-blue (RGB), multispectral, hyperspectral, and thermal cameras, stereo cameras; and light detection and ranging (LiDAR) devices. These sensors occupy different ranges of wavelengths in the Electromagnetic spectrum (EMS), as shown in Figure 1-2. Although with some overlaps, these sensors varied in bandwidth, image resolution, image quality and price. They have been used in many studies to sense crop traits for different purposes.

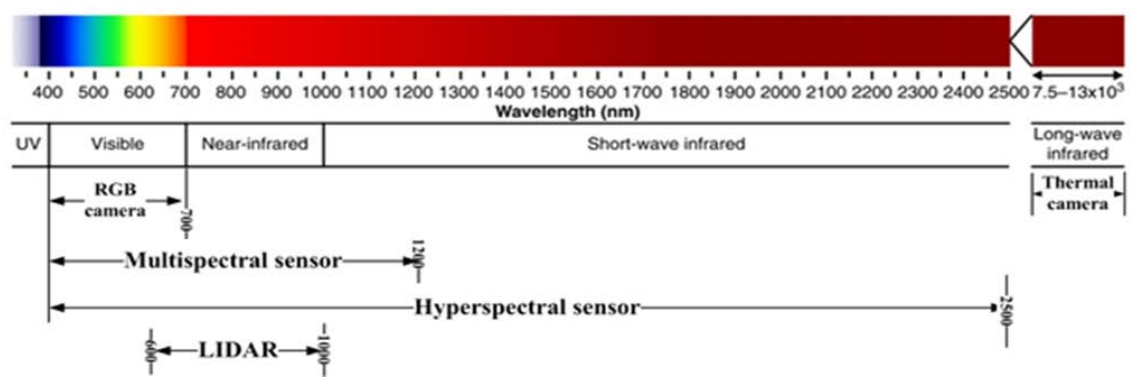

Figure 1-2. Electromagnetic spectrum (EMS) and sensors corresponding to different regions of wavelength (Fahlgren et al., 2015).

RGB cameras record red (564-580 nm), green (534-545 nm), and blue (420-440 nm) spectral bands in the visible EMS regions. to produce digital images and mimic human visions (Jin et al., 2017). They are capable to obtain color information, 2D geometric information (e.g., canopy area) and depth information (e.g., plant height) from crop canopy. RGB cameras are the most commonly adopted sensors in the HTP platforms due to their advantages of low cost, high-resolution, user-friendly operation, light weight and adaptability to the working environment (Yang et al., 2017). However, three spectral bands and their broad bandwidth of RGB cameras limit their ability to receive crop traits other 
than human perceptions, and their pixel digital numbers (DNs) are largely dependent on environmental radiations, thus hardly being used to estimate plant traits without preprocessing.

Multispectral and hyperspectral cameras extend the sensing range of RGB cameras with narrower and more bands along with the EMS. Multispectral cameras commonly include several spectral bands in the infrared spectral regions that have been shown sensitive to leaf pigments, for example, the red-edge region that is the transition from the visible to the near-infrared (NIR) spectral region (Geipel et al., 2016). While hyperspectral cameras have a higher spectral resolution, with continuous or discrete spectral bands (Jay et al., 2017), enable calculating more advanced vegetation indices (VIs) and unperceived crop phenotypes. The multi-and hyper-spectral cameras often require serial steps of imaging and pre-processing, including calibration radiometric calibration to reduce effects of light variations, image stitching and alignment (Zarco-Tejada et al., 2012).

Thermal cameras are used to capture the long-wave infrared spectral radiation emitted from crops and convert such reflectance to an electrical signal which highly correlates with the surface temperature of crops. Such surface temperature can be an indicator of its evaporation or transpiration rates. When a plant experiences abiotic or biotic stresses, these rates will change as well as its surface temperature. Hence, thermal cameras are widely used to detect such stresses and also evaluate the responses of plants with tolerant or susceptible abilities to the stresses (Gago et al., 2015; Zhou et al., 2020).

Stereo cameras and LiDAR devices are generally used to obtain depth information of targeted objects. Stereo cameras are very similar to the traditional RGB camera but with two or more lenses. Given two sets of two-dimensional (2D) images from both lenses, 
three-dimensional information can be generated using stereoscopy techniques that take advantage of the parallax characteristics of human eyes (W. Li et al., 2016). A LiDAR device has a single-beam laser (narrowband in 600-1000 nm) that emits a light pulse and a receiver measures the propagation time of the light pulse from emission to reflection. The time is then converted into a distance given the known speed of light (Guo et al., 2018).

(3) Analytical methods and models

The HTP platforms and sensors provide multi-dimensional data to characterize crop phenotypic traits across a spatial and temporal scale on hundreds or thousands of breeding materials. Those data for different research purposes require dedicated methods to remove irrelevant or redundant information and tune model arguments to eventually reach acceptable results. Although with excellent interpretability, traditional regression-based models are limited by their model complexity to explain multivariate relationships between the predictors and responses (Parmley et al., 2019). Over the past few years, with access to high-performing computers and Application Programming Interfaces (APIs, for example, Keras in Python), machine learning (ML) and deep learning (DL) models have been extensively applied in crop breeding problems with HTP inputs.

Classic supervised ML methods, such as support vector machine, tree-based models and Linear/Quadratic Discriminant Analysis are mostly fit to estimate continuous crop traits (e.g., yield and plant height) and classify categorical traits (e.g., stress injury scores and maturity groups). Selecting an appropriate set of input features to these models is critical to the classification or prediction performance (Chandrashekar \& Sahin, 2014). Therefore, an unsupervised ML method, Principal Component Analysis (PCA), is often used to reduce feature dimensions by creating principal components (linear combinations 
of relevant features) that explain the most variations in the dataset. Others supervised ML model, i.e., the Lasso (least absolute shrinkage and selection operator) and ridge regression, could also help to shrink the dataset by assigning extremely small coefficients (even 0) to the variables that had little contributions to the model performance (James et al., 2013).

DL is the most important branch of ML allowing hierarchical data learning. One of the advantages of DL is that it does not require an extra step for feature selection, instead, important features will be discriminated during the training process by adjusting a set of parameters associated with the input features. There are a few classic DL models widely applied in HTP, including artificial neural networks (ANNs) taking tabulated input similar to ML models, convolutional neural networks (CNNs) for image analysis, recurrent neural networks (RNNs) for sequence or time data processing, and auto-encoder networks (AENs) (Goodfellow et al., 2016). In terms of disadvantages, however, DL models require a large dataset for training, testing, and validation. They are also computationally expansive and have poor interpretability (L. Zhang et al., 2016).

\subsubsection{The applications of UAV-based HTP in crop breeding}

In the application of HTP for crop breeding, UAV platforms have been applied in many studies due to its proper balance between data collection efficiency and quality, easy operation and flexibility to carry various types of devices (e.g., sensors, power sources, GNSS units, data storages and processing units). It has been witnessed to become an essential method for quickly obtaining crop traits for various purposes (Näsi et al., 2018). The section summarizes the applications of UAV-based HTP in obtaining crop traits that are commonly considered as primary and secondary traits for breeding selections, including plant height, lodging, flower, maturity, stress and yield. 


\section{(1) Plant height and lodging}

Plant height is one of the most important secondary traits for the breeders. Plant height usually had a significantly positive correlation with yield in many types of crops, thus it is often used as an indicator of yield potential (Feng et al., 2019; W. Li et al., 2016; Wan et al., 2019; Yue et al., 2017). Besides, plant height can be used to detect crop lodging that is observed as the permanent deviation of crop plants from the upright position (Pinthus, 1974). Lodging reduces harvest efficiency and increases yield losses (Fischer \& Stapper, 1987).

Various sensors and methods have been developed to rapidly and accurately measure plant height, as the conventionally manual measurements are very timeconsuming and labor-intensive. There are a few applications of crop phenotyping using LiDAR systems to estimate plant height and biomass due to high point density, high spatial resolution, lighter weight, and good performance in low-altitude detection. Walter et al. (2018) reported an $R^{2}$ of 0.94 with an RMSE of $11.5 \mathrm{~cm}$ between the LiDAR height measurements on 173 maize hybrid lines and manual measurements.

One of the most popular stereovision techniques, Structure from Motion (SfM) enables the reconstruction of digital elevation models (DEMs) from a series of overlapping 2D RGB images. Similar to LiDAR, the DEM is in a format of dense point cloud with each point represented by a position in a local coordinate (x, y, and z axis) or WGS84 (World Geodetic System 1984, i.e., latitude, longitude, and altitude) and a color (red, green, and blue). Zhou et al. (2021) reported an RMSE of $2.9 \mathrm{~cm}$ for 12 GCPs (99 cm high) and a relative $R M S E$ of $6.1 \%$ (RMSE $=3.6 \mathrm{~cm}$ ) for soybean plots between the manually measured height and estimated height from a DEM generated by a set of RGB images 
collected $15 \mathrm{~m}$ above ground in a soybean breeding field.

\section{(2) Stresses}

Plants may suffer various kinds of stress conditions during their growth, including abiotic stresses in adverse environments, such as drought, flooding, salt, heat and chill, etc., and biotic stresses, such as weed stress, pests, and disease stress (Abdel-Haleem et al., 2012). The stresses have significant adverse effects on plant growth and could reduce plant yield ultimately. Studying the response of plants to different stress conditions is essential to cultivating stress tolerant varieties in crop breeding (Valliyodan et al., 2016).

Plant canopy reflectance can be used as an indicator of plant health status under stress conditions because the reflected light directly depends on leaf pigment compositions (e.g., chlorophylls) (Jones \& Vaughan, 2010). The reflectance in specific spectral bands could be captured by RGB, multispectral and hyperspectral cameras and then used to develop various VIs. One of the most widely used VIs is the Normalized Difference Vegetation Index (NDVI) that has been used to detect vegetations and evaluate plant 'greenness' (Gago et al., 2015):

$$
N D V I=\frac{N I R-R}{N I R+R}
$$

where NIR is the reflectance in the near-infrared band and $R$ in the red band. Other imagederived VIs and geometric features were also investigated to detect plant physiological responses under stress. For example, drought is one of the most studied stress as its limiting factors in crop growth and production. It was reported that the yield of wheat, maize and soybean reduced $21 \%, 40 \%$ and $40 \%$, respectively, due to drought on a global scale (Daryanto et al., 2016). Zhou et al. (2020) evaluated the differences in a group of VIs and 
canopy temperatures between drought tolerant and sensitive soybean genotypes and the results show that drought tolerant genotypes had significantly ( $p$-value $<0.01$ ) higher NDVI, hue, saturation, canopy size, height, and lower temperature than drought sensitive genotypes. Those image traits were also shown to have higher broad-sense heritability $\left(\mathrm{H}^{2}\right)$ than yield.

\section{(3) Maturity}

Maturity time stands for the time length of soybean from planting to their physiological maturity. For some particular crop types, maturity time is a critical characteristic for breeders to select the crop genotypes with desired traits. Taking soybean as an example, soybeans with extended maturity time may take full advantage of the growing season to improve their yield (Staton, 2017). However, soybeans in delayed harvest days may suffer frost damages or high harvest losses. Therefore, a unique attributematurity group was given to crop whose productions related to their maturity time. For soybean, there are 10 maturity groups (MGs) from 0 to 9 according to their time length from planting to physiological maturity. MG 0 has the shortest maturity time and 9 has the longest maturity time. MG zones were developed accordingly to define where a soybean cultivar grows best (Mourtzinis \& Conley, 2017).

Breeders need to figure out the maturity group of their new varieties and select those suitable for their targeted climate. To determine the MG of a new variety, breeders compare maturity dates of the new variety with checks that are in known MGs. Maturity date is the first day of the year when soybeans reach physiological maturity status. A soybean is considered mature when $95 \%$ of its pods achieve mature color. Conventionally, soybean breeders take notes of maturity date by visual observations. With tens of 
thousands of breeding lines in a season, it is very tedious to take notes of every single lines.

Field HTP could be used to capture the difference between mature and immature plots and use the difference to predict the maturity date/MGs of breeding materials. Yu et al. (2016) developed a random forest model to classify mature and immature soybean lines using images in the blue and NIR band from a UAV-based HTP platform and achieved over 93\% classification accuracy. Christenson et al. (2016) predicted the relative maturity of soybean lines using a PLSR model with three VIs derived from narrow spectral bands that were acquired by a UAV imaging system. The correlation between predicted relative maturity and observed maturity was 0.5 with an RMSE of 5.19 days.

(4) Yield estimation

Yield is the primary trait for selecting elite breeding genotypes, but a very complicated crop trait. Although the potential of estimating crop yield using image features has been reported in many studies, there are few studies conducted to predict the yield of soybean breeding lines in early breeding cycles. In breeding programs, tens of thousands of crosses from two parents are planted in progeny trials without replicates. Large variations in phenotypes (e.g. yield) can be caused by the conjunctions of their genetic and environmental variances (Fehr, 1991), consequently bringing large variances in image features and model predictions. Although a small percentage of selected genotypes are planted in following multi-location yield trials, the expansion of field networks across geographic locations increases spatial variances and consequently phenotypic variances in the same genotypes (Araus et al., 2018).

The most widely used method for yield estimation is linear regression that has high interpretability and shows good performance in some cases. Advanced data analytic 
methods, such as ML and DL, show great potential in processing complicated data sets and extracting hidden information of data that are not easily acquired using restrictive models (Elavarasan et al., 2018). Maimaitijiang et al. (2020) predicted the yield of three soybean cultivars under two irrigation treatments with an $R^{2}$ of 0.72 and an RMSE of $1054.9 \mathrm{~kg} \cdot \mathrm{ha}^{-}$

${ }^{1}$ using a deep neural networks (DNN) based on RGB, multispectral, and thermal images. Ashapure et al. (2019) reported a result of $R^{2}=0.7-0.89$ for predicting the yield of a set of tomato genotypes using a simple ANN model based on UAV-based RGB images.

\subsubsection{Limitations and Challenges}

Obtaining accurate and inexpensive estimates of the genetic value of breeding materials is central to improve breeding efficiency toward increasing genetic gain (Araus et al., 2018). While plant breeders have started using high-resolution genome information for germplasm characterization and genetic dissection of major agronomic traits, phenotypic traits such as maturity date and yield remain largely manual within breeding programs. Thus, the research bottleneck in crop breeding is shifting from genotyping to phenotyping (Mir et al., 2019). However, there are challenges limiting the adoption of the HTP advances for breeding purposes.

Data acquisition, processing, management, interpretation and modeling form the core of HTP to deliver accurate measurements of plant traits (D. Li et al., 2021). Each of the steps, more or less, currently requires manual interactions from those who have specific skills, such as remotely operating UAVs, programming, and statistical analyzing, etc. Although various software, tools, and online database have emerged to assist users with one or more steps in HTP pipelines, such as Coverage Tool, Plant 3D and Quantitative Plan, it is still being challenging for breeders to apply and customize these tools for their 
specific research purposes. Thus, more user-friendly or 'breeders preferred' platforms are still needed to bridge the gap between breeders and the research community of 'phenotypers'.

Besides, it is well noted that the current research in the field HTP largely focuses on developing HTP platforms, selecting input features and models, and validating the relationship between HTP data and ground data. When the platforms and models are under real breeding scenarios, they might not be as effective as they were tested due to large variations in field environmental conditions, breeding materials and their interactions. Therefore, there is an urgent need to integrate the current advances into a pipeline, gathering both image and ground data from yearly trials and training models to adapt to these variations, and eventually touching the goal of breeding programs to make selections of elite genotypes toward increased genetic gain (Araus et al., 2018).

\subsection{Goals and objectives}

Motivated by the need to bridge the gap between breeders and 'phenotypers', the goal of this research was to develop an integrated and automated HTP framework leveraging advanced technologies in remote sensing and artificial intelligence for estimating key traits and selecting elite genotypes towards improving the selection intensity and accuracy of conventional soybean breeding. To achieve this goal, this study features three objectives:

Objective 1: Develop an integrated and automated UAV HTP framework for measuring crop traits accurately and efficiently. The framework integrates a hardware module to acquire high-quality aerial images with accurate geo-references, as well as an applicational software module to automatedly process the multi-dimensional images. The 
framework can generate reliable measurements of plant geometric and morphological traits for further analysis.

Objective 2: Estimate soybean yield and maturity date of breeding materials using UAV image features and machine learning models. Yield is the primary trait for selecting elite soybean lines and the maturity date is decisive to select suitable lines for particular geographical regions. Accurate estimations of these two agronomic traits will increase the breeding population size that has been limited by the requirement of human input in field evaluations, and therefore increase the probability of selecting elite lines. Such estimations early in plant development allow selection decisions to be made before completing a growing season, eventually beneficial to saving breeding resources and reducing breeding circles.

Objective 3: Select elite soybean lines using UAV image features. The primary goal of soybean breeding is to select elite lines with high yield potential, meanwhile taking into consideration a few secondary traits, such as maturity date and logding, etc. Additional to estimating the primary and secondary traits, the HTP framework can make selections on soybean lines by learning from the past breeder's selections and rank the candidate lines. With the initiative HTP suggestions on elite lines, breeders will be able to select a smaller group with higher yield potential to increase the selection intensity and accuracy towards improving genetic gain.

\subsection{Dissertation outlines}

The dissertation has five chapters:

Chapter One: Introduction. This chapter describes the background and justifications 
to this research and a literature review of current HTP developments and their applications in crop breeding. It also listed the research goal and objectives, and an outline of the dissertation.

Chapter Two: Estimation of the maturity date of soybean breeding lines using UAV-based multispectral imagery. The physiological maturity date is a critical parameter for the selection of breeding lines in soybean breeding programs. This chapter was to investigate the potential of predicting maturity dates of soybean breeding lines using UAVbased multispectral imagery.

Chapter Three: Yield estimation of soybean breeding lines under drought stress using UAV-based imagery and CNN. This chapter was to evaluate the performance of a UAV imaging system and deep learning in estimating the yield of soybean breeding lines. A group of soybean breeding lines in three maturity groups was planted under rainfed conditions for testing their drought tolerance.

Chapter Four: Variety selection using UAV HTP towards improving selection accuracy and intensity for soybean breeding. This chapter was to model the breeder's selection using a group of agronomic traits and investigate the potential of selecting superior soybean varieties for soybean breeding programs using image-based secondary traits. The model selection was evaluated regarding selection and accuracy (future yield performance) and intensity (small group of selected genotypes with higher yield).

Chapter Five: Conclusion and future work. A summary of the results in this dissertation research and expectations for future work were presented in chapter five. 


\section{Reference}

Abdel-Haleem, H., Carter, T. E., Purcell, L. C., King, C. A., Ries, L. L., Chen, P., et al. (2012). Mapping of quantitative trait loci for canopy-wilting trait in soybean (Glycine max L. Merr). Theoretical and Applied Genetics, 125(5), 837-846. 10.1007/s00122-012-1876-9

Ahmar, S., Gill, R. A., Jung, K.-H., Faheem, A., Qasim, M. U., Mubeen, M., et al. (2020). Conventional and Molecular Techniques from Simple Breeding to Speed Breeding in Crop Plants: Recent Advances and Future Outlook. International journal of molecular sciences, 21(7), 2590. 10.3390/ijms21072590

Araus, J. L., \& Cairns, J. E. (2014). Field high-throughput phenotyping: the new crop breeding frontier. Trends in Plant Science, 19(1), 52-61

Araus, J. L., Kefauver, S. C., Zaman-Allah, M., Olsen, M. S., \& Cairns, J. E. (2018). Translating high-throughput phenotyping into genetic gain. Trends in Plant Science, 23(5), 451-466. https://doi.org/10.1016/j.tplants.2018.02.001

Ashapure, A., Oh, S., Marconi, T. G., Chang, A., Jung, J., Landivar, J., et al. (2019). Unmanned aerial system based tomato yield estimation using machine learning. Paper presented at the Proceedings of SPIE - The International Society for Optical Engineering. https://doi.org/10.1117/12.2519129

BATS Center, B. a. S. (1995). Methods of Plant Breeding. Retrieved from https://www.bats.ch/bats/publikationen/1995-1_TA/2-methods.php

Blum, A. (2014). Genomics for drought resistance - getting down to earth. Functional Plant Biology, 41(11), 1191-1198. https://doi.org/10.1071/FP14018

Chandrashekar, G., \& Sahin, F. (2014). A survey on feature selection methods. Computers \& Electrical Engineering, 40(1), 16-28

Christenson, B. S., Schapaugh, W. T., An, N., Price, K. P., Prasad, V., \& Fritz, A. K. (2016). Predicting Soybean Relative Maturity and Seed Yield Using Canopy Reflectance. Crop Science, 56(2), 625-643. 10.2135/cropsci2015.04.0237

Daryanto, S., Wang, L., \& Jacinthe, P.-A. (2016). Global synthesis of drought effects on 
maize and wheat production. PLoS One, 11(5)

Dwivedi, S. L., Goldman, I., Ceccarelli, S., \& Ortiz, R. (2020). Chapter Three Advanced analytics, phenomics and biotechnology approaches to enhance genetic gains in plant breeding. In D. L. Sparks (Ed.), Advances in Agronomy (Vol. 162, pp. 89-142): Academic Press.

Elavarasan, D., Vincent, D. R., Sharma, V., Zomaya, A. Y., \& Srinivasan, K. (2018). Forecasting yield by integrating agrarian factors and machine learning models: A survey. Computers and Electronics in Agriculture, 155, 257-282. https://doi.org/10.1016/j.compag.2018.10.024

Fahlgren, N., Feldman, M., Gehan, Malia A., Wilson, Melinda S., Shyu, C., Bryant, Douglas W., et al. (2015). A Versatile Phenotyping System and Analytics Platform Reveals Diverse Temporal Responses to Water Availability in Setaria. Molecular Plant, 8(10), 1520-1535. https://doi.org/10.1016/j.molp.2015.06.005

Fasoula, D. A., Ioannides, I. M., \& Omirou, M. (2020). Phenotyping and Plant Breeding: Overcoming the Barriers. 10(1713). 10.3389/fpls.2019.01713

Fehr, W. R. (1991). Principles of cultivar development: theory and technique. New York, NY: Macmillian.

Feng, A., Zhang, M., Sudduth, K. A., Vories, E. D., \& Zhou, J. (2019). Cotton yield estimation from UAV-based plant height. Transactions of the ASABE, 62(2), 393-404. https://doi.org/10.13031/trans.13067

Fiorani, F., \& Schurr, U. (2013). Future scenarios for plant phenotyping. Annual review of plant biology, 64, 267-291

Fischer, R., \& Stapper, M. (1987). Lodging effects on high-yielding crops of irrigated semidwarf wheat. Field Crops Research, 17(3-4), 245-258

Fu, Y.-B., Yang, M.-H., Zeng, F., \& Biligetu, B. (2017). Searching for an Accurate Marker-Based Prediction of an Individual Quantitative Trait in Molecular Plant Breeding. Frontiers in Plant Science, 8(1182). 10.3389/fpls.2017.01182

Furbank, R. T., \& Tester, M. (2011). Phenomics - technologies to relieve the 
phenotyping bottleneck. Trends in Plant Science, 16(12), 635-644.

https://doi.org/10.1016/j.tplants.2011.09.005

Gago, J., Douthe, C., Coopman, R. E., Gallego, P. P., Ribas-Carbo, M., Flexas, J., et al. (2015). UAVs challenge to assess water stress for sustainable agriculture. Agricultural Water Management, 153, 9-19. https://doi.org/10.1016/j.agwat.2015.01.020

Geipel, J., Link, J., Wirwahn, J. A., \& Claupein, W. (2016). A programmable aerial multispectral camera system for in-season crop biomass and nitrogen content estimation. Agriculture, 6(1), 4

Goodfellow, I., Bengio, Y., \& Courville, A. (2016). Deep learning: MIT press.

Guo, Q., Wu, F., Pang, S., Zhao, X., Chen, L., Liu, J., et al. (2018). Crop 3D—a LiDAR based platform for 3D high-throughput crop phenotyping. Science China Life Sciences, 61(3), 328-339

James, G., Witten, D., Hastie, T., \& Tibshirani, R. (2013). An introduction to statistical learning (Vol. 112): Springer.

Jay, S., Maupas, F., Bendoula, R., \& Gorretta, N. (2017). Retrieving LAI, chlorophyll and nitrogen contents in sugar beet crops from multi-angular optical remote sensing: Comparison of vegetation indices and PROSAIL inversion for field phenotyping. Field Crops Research, 210, 33-46

Jiang, G.-L. (2013). Molecular markers and marker-assisted breeding in plants. Plant breeding from laboratories to fields, 45-83

Jin, X., Liu, S., Baret, F., Hemerlé, M., \& Comar, A. (2017). Estimates of plant density of wheat crops at emergence from very low altitude UAV imagery. Remote Sensing of Environment, 198, 105-114

Jin, X., Zarco-Tejada, P. J., Schmidhalter, U., Reynolds, M. P., Hawkesford, M. J., Varshney, R. K., et al. (2021). High-Throughput Estimation of Crop Traits: A Review of Ground and Aerial Phenotyping Platforms. IEEE Geoscience and Remote Sensing Magazine, 9(1), 200-231. 10.1109/MGRS.2020.2998816 
Jones, H. G., \& Vaughan, R. A. (2010). Remote sensing of vegetation: principles, techniques, and applications: Oxford university press.

Krueger, S. (2019). How Breeders Develop New Soybean Varieties. Retrieved from https://emergence.fbn.com/inputs/how-breeders-develop-new-soybean-varieties

Li, D., Quan, C., Song, Z., Li, X., Yu, G., Li, C., et al. (2021). High-Throughput Plant Phenotyping Platform (HT3P) as a Novel Tool for Estimating Agronomic Traits From the Lab to the Field. Frontiers in Bioengineering and Biotechnology, 8(1533). 10.3389/fbioe.2020.623705

Li, W., Niu, Z., Chen, H., Li, D., Wu, M., \& Zhao, W. (2016). Remote estimation of canopy height and aboveground biomass of maize using high-resolution stereo images from a low-cost unmanned aerial vehicle system. Ecological Indicators, 67, 637-648. https://doi.org/10.1016/j.ecolind.2016.03.036

Lorenz, A. J., Chao, S., Asoro, F. G., Heffner, E. L., Hayashi, T., Iwata, H., et al. (2011). Genomic Selection in Plant Breeding: Knowledge and Prospects. In D. L. Sparks (Ed.), Advances in Agronomy (Vol. 110, pp. 77-123): Academic Press.

Maimaitijiang, M., Sagan, V., Sidike, P., Hartling, S., Esposito, F., \& Fritschi, F. B. (2020). Soybean yield prediction from UAV using multimodal data fusion and deep learning. Remote Sensing of Environment, 237, 111599. https://doi.org/10.1016/j.rse.2019.111599

Mammadov, J., Aggarwal, R., Buyyarapu, R., \& Kumpatla, S. (2012). SNP Markers and Their Impact on Plant Breeding. International Journal of Plant Genomics, 2012, 11. $10.1155 / 2012 / 728398$

Mir, R. R., Reynolds, M., Pinto, F., Khan, M. A., \& Bhat, M. A. (2019). High-throughput phenotyping for crop improvement in the genomics era. Plant Science, 282, 6072. https://doi.org/10.1016/j.plantsci.2019.01.007

Moreira, F. F., Hearst, A. A., Cherkauer, K. A., \& Rainey, K. M. (2019). Improving the efficiency of soybean breeding with high-throughput canopy phenotyping. Plant methods, 15(1), 139. https://doi.org/10.1186/s13007-019-0519-4

Mourtzinis, S., \& Conley, S. P. (2017). Delineating Soybean Maturity Groups across the 
United States. Agronomy Journal, 109, 1397-1403. 10.2134/agronj2016.10.0581

Näsi, R., Viljanen, N., Kaivosoja, J., Alhonoja, K., Hakala, T., Markelin, L., et al. (2018). Estimating Biomass and Nitrogen Amount of Barley and Grass Using UAV and Aircraft Based Spectral and Photogrammetric 3D Features. Remote Sensing, 10(7), 1082

Parmley, K. A., Higgins, R. H., Ganapathysubramanian, B., Sarkar, S., \& Singh, A. K. (2019). Machine Learning Approach for Prescriptive Plant Breeding. Scientific Reports, 9(1), 17132. 10.1038/s41598-019-53451-4

Pinthus, M. J. (1974). Lodging in wheat, barley, and oats: the phenomenon, its causes, and preventive measures Advances in agronomy (Vol. 25, pp. 209-263): Elsevier.

Shu, Q., \& Wu, D. (2016). Rice Breeding Reference Module in Food Science: Elsevier.

Sleper, D. A., \& Poehlman, J. M. (2006). Breeding field crops: Blackwell publishing.

Staton, M. (2017). What is the relationship between soybean maturity group and yield? Retrieved from https://www.canr.msu.edu/news/what_is_the_relationship_between_soybean_mat urity_group_and_yield

Valliyodan, B., Ye, H., Song, L., Murphy, M., Nguyen, H. T., \& Shannon, J. G. (2016). Genetic diversity and genomic strategies for improving drought and waterlogging tolerance in soybeans. Journal of Experimental Botany, 68(8), 1835-1849. https://doi.org/10.1093/jxb/erw433

Walter, J., Edwards, J., McDonald, G., \& Kuchel, H. (2018). Photogrammetry for the estimation of wheat biomass and harvest index. Field Crops Research, 216, 165174. 10.1016/j.fcr.2017.11.024

Wan, L., Cen, H., Zhu, J., Li, Y., Zhu, Y., Sun, D., et al. (2019). Combining UAV-based vegetation indices, canopy height and canopy coverage to improve rice yield prediction under different nitrogen levels. Paper presented at the 2019 ASABE Annual International Meeting. 10.13031/aim.201900626

Xu, Y., Li, P., Zou, C., Lu, Y., Xie, C., Zhang, X., et al. (2017). Enhancing genetic gain 
in the era of molecular breeding. Journal of Experimental Botany, 68(11), 26412666. 10.1093/jxb/erx135

Yang, G., Liu, J., Zhao, C., Li, Z., Huang, Y., Yu, H., et al. (2017). Unmanned aerial vehicle remote sensing for field-based crop phenotyping: Current status and perspectives. Frontiers in Plant Science, 8, 1111. 10.3389/fpls.2017.01111

Yu, N., Li, L., Schmitz, N., Tian, L. F., Greenberg, J. A., \& Diers, B. W. (2016). Development of methods to improve soybean yield estimation and predict plant maturity with an unmanned aerial vehicle based platform. Remote Sensing of Environment, 187, 91-101. https://doi.org/10.1016/j.rse.2016.10.005

Yue, J., Yang, G., Li, C., Li, Z., Wang, Y., Feng, H., et al. (2017). Estimation of winter wheat above-ground biomass using unmanned aerial vehicle-based snapshot hyperspectral sensor and crop height improved models. Remote Sensing, 9(7). $10.3390 /$ rs9070708

Zarco-Tejada, P. J., González-Dugo, V., \& Berni, J. A. (2012). Fluorescence, temperature and narrow-band indices acquired from a UAV platform for water stress detection using a micro-hyperspectral imager and a thermal camera. Remote Sensing of Environment, 117, 322-337

Zhang, C., Marzougui, A., \& Sankaran, S. (2020). High-resolution satellite imagery applications in crop phenotyping: An overview. Computers and Electronics in Agriculture, 175, 105584. https://doi.org/10.1016/j.compag.2020.105584

Zhang, L., Zhang, L., \& Du, B. (2016). Deep Learning for Remote Sensing Data: A Technical Tutorial on the State of the Art. IEEE Geoscience and Remote Sensing Magazine, 4(2), 22-40. 10.1109/MGRS.2016.2540798

Zhou, J., Zhou, J., Ye, H., Ali, M. L., Chen, P., \& Nguyen, H. T. (2021). Yield estimation of soybean breeding lines under drought stress using unmanned aerial vehiclebased imagery and convolutional neural network. Biosystems Engineering, 204, 90-103. https://doi.org/10.1016/j.biosystemseng.2021.01.017

Zhou, J., Zhou, J., Ye, H., Ali, M. L., Nguyen, H. T., \& Chen, P. (2020). Classification of soybean leaf wilting due to drought stress using UAV-based imagery. Computers 
and Electronics in Agriculture, 175, 105576.

https://doi.org/10.1016/j.compag.2020.105576 


\section{CHAPTER TWO}

\section{ESTIMATION OF THE MATURITY DATE OF SOYBEAN BREEDING LINES USING UAV-BASED MULTISPECTRAL IMAGERY}

\subsection{Abstract}

Physiological maturity date is a critical parameter for the selection of breeding lines in soybean breeding programs. The conventional method to estimate the maturity dates of breeding lines uses visual ratings of the pod senescence by experts, which is subjective by human bais, labor-intensive and time-consuming. Unmanned aerial vehicle (UAV)-based phenotyping systems provide a high-throughput and powerful tool to capture crop traits using remote sensing, image processing and machine learning technologies. The goal of this study was to investigate the potential of predicting maturity dates of soybean breeding lines using UAV-based multispectral imagery. Maturity dates of 326 soybean breeding lines were taken using visual ratings from the beginning maturity stage (R7) to full maturity stage (R8), and the aerial multispectral images were taken during this period on Aug. 27, Sept. 14, and 27, 2018. One hundred and thirty features were extracted from the five-band multispectral images. The maturity dates of the soybean lines were predicted and evaluated using partial least square regression (PLSR) models with 10-fold cross-validation. Twenty image features with importance to the estimation were selected and their changing rates between each two of the data collection days were calculated. The best prediction $\left(R^{2}=\right.$ 0.81, RMSE $=1.4$ days) was made by the PLSR model using image features taken on Sept.14 and their changing rates between Sept. 14 and 27 with five components, leading to the conclusion that the UAV-based multispectral imagery is promising and practical in estimating maturity dates of soybean breeding lines. 


\subsection{Introduction}

By 2050, the global population is expected to reach 9.8 billion (Hincks, 2018) and the current arable land is decreasing due to climate change, urbanization, soil degradation, water shortages and pollution (Breene, 2018). Food demand is expected to be $60 \%$ higher than it is today, resulting in limited global food supplies. Crop breeding is a promising solution for the food crisis by developing new crop varieties with improved traits, including high yield potential and resilience to biotic and abiotic stresses due to adverse environments (Sun, 2014).

The rationale of breeding programs is to select among a large number of genotypes crop varieties with high yield potential, tolerance to biotic and abiotic stresses and high efficiency in cultivation, harvest and processing (Breseghello \& Coelho, 2013). Selection criteria usually include yield, lodging, flowering, maturity date, stress symptoms, severity, etc., with different emphasis depending on the purpose of breeding programs. Specifically, in soybean breeding programs, the physiological maturity date is a critical parameter for selecting soybean lines. Soybeans with extended maturity time may take full advantage of the growing season to improve their yield (Staton, 2017). However, soybeans in delayed harvest days may suffer frost damages or high harvest losses. Therefore, soybean cultivars are divided into maturity groups (MGs, e.g., MG III or MG IV) from 0 to 9 according to their time length from planting to the physiological maturity. MG 0 has the shortest maturity time and 9 has the longest maturity time. MG zones were developed to define where a soybean cultivar grows best (Mourtzinis \& Conley, 2017).

Breeders determine the MG of a new variety by referring to the relative maturity (RM, by dividing an MG into 10 subgroups, e.g., RM3.0 to RM3.9 are 10 RMs in MG III) 
of the new variety to commercially released soybean cultivars (referred to as checks) with a known MG. The RM is determined by the differences in the maturity dates (the first day of the year when soybeans reach maturity status) between the new variety and the checks. Conventionally, soybean breeders take measurements of the physiological maturity status of desired breeding lines using visual ratings by observing the senescence color of soybean pods. However, the visual rating is labor-intensive, time-consuming, and more importantly, it is subjective (Masuka et al., 2017). In conventional breeding programs, a breeder may need to scout tens of thousands of breeding lines in a progeny trial in a season (Yu et al., 2016), which makes it challenging to select the most desired lines. Therefore, there is a pressing need to develop an efficient and effective tool to take measurements of the maturity date of soybean progeny breeding lines in fields using novel approaches.

Maturity generally occurs when soybean pods have reached their physiological matured stages, showing gray, tan, or brown color in pods (Fehr et al., 1971). After entering the beginning maturity stage (R7), a soybean plant, including leaves and pods, loses its moisture quickly from around $60 \%$ to less than $30 \%$, depending on the local air temperature, humidity, and soil conditions (Peske et al., 2004). In addition, with the plant approaching senescence, the chlorophyll content of leaves reduces and the color turns to yellow/gray (Fehr et al., 1971). This change might result in substantial changes in spectral reflectance. Therefore, it is possible to quantify the plant maturity level using the combinations of spectral reflectance in given wavebands that are very sensitive to crop canopy characteristics, such as chlorophyll content (Gitelson et al., 2003), nitrogen content or water status (Barnes et al., 2000).

Yu et al. (2016) developed a random forest model to classify mature and immature 
soybean lines using images in the blue and near-infrared bands from an unmanned aerial vehicle (UAV)-based high-throughput phenotyping (HTP) platform, and achieved over 93\% classification accuracy. Christenson et al. (2016) predicted the RM of soybean lines using a partial least square regression (PLSR) model with three vegetation indices (VIs) derived from narrow spectral bands that were acquired by a UAV imaging system. The correlation of determination $\left(R^{2}\right)$ between the predicted RM and observed maturity was 0.5 with a root mean square error (RMSE) of 5.19 days. However, the difference between two relative maturity designations (e.g., RM 3.7 and RM 3.8) is usually only one to two days (personal communication with a soybean breeder-Dr. Andrew Scaboo). It is not sufficient for soybean breeding purposes by classifying breeding lines into only two classes of mature and immature lines or the prediction with 5 days error. To the best of our knowledge, there is no such study showing the ability to determine accurate maturity date for breeders.

Therefore, the goal of this study was to investigate a method to estimate the maturity dates of soybean breeding lines using a UAV-based imaging system. There were three supportive objectives to achieve this goal: (1) To investigate the best time (growth stage) to collect images, (2) to select image features important to estimate relative maturity date, and (3) to evaluate the accuracy of our estimates.

2.3 Materials and methods

\subsubsection{Field experiment}

The field experiment was conducted at an experimental field in the Greenley Research Center of the University of Missouri, Novelty, Missouri, United States ( $40^{\circ} 01^{\prime} \mathrm{N}$, $92^{\circ} 11^{\prime} \mathrm{W}$ ) in 2018. Eleven thousand, four hundred and seventy-three soybean lines were inbred from soybean varieties in MG III and IV, and planted on May 29, 2018, in a 3.64 ha 
field. The soybean lines were planted in single-row plots, each being a progeny row derived from a single F4 plant, without replicates with $2.59 \mathrm{~m}$ in length and $0.76 \mathrm{~m}$ spacing in between rows. The breeding lines started maturing from Sept. 19 and were completed on Oct. 2, 2018.

Three hundred and twenty-six soybean lines were randomly selected before R8 stages as ground references for the estimation of maturity dates. The maturity dates of these soybean lines were determined by an experienced breeder using visual assessments. The measurements of maturity date were recorded every seven days starting from Aug. 31 to Oct. 5. Mature plots were not observed until Sept. 19. In each measuring day, a soybean line that has approximately $95 \%$ of its pods achieved mature pod color was determined to be mature and the date was recorded as its maturity date, and for those that have slightly less or more than $95 \%$ pods achieved mature color, the maturity dates were estimated as 1 to 3 days after or before the measuring day.

\subsubsection{UAV data collection}

Multispectral images were acquired using a multispectral camera RedEdge-M (MicaSense, Seattle, WA, USA) that has a resolution (number of total pixels) of $1260 \times$ 960 pixels. The details of wavelength are shown in Table 2-1. An iPad mini 3 (Apple Inc., Cupertino, CA, USA) was used to configure the camera taking time-lapse images at 1 frame per second (fps) by connecting the camera using its Wi-Fi hotspot. A GNSS (Global Navigation Satellite System) unit was attached to the camera and pre-programed to provide geo-referencing information for each frame of images. All images with the EXIF (Exchangeable Image File Format) metadata were saved to an SD card on the camera. Before each flight, a calibration reflectance panel (CRP) was imaged by holding the camera 
at about $1 \mathrm{~m}$ above the CRP and looking vertically in an open area (to avoid shadow). The CRP has a factory calibrated reflectance in each of the five spectral bands and is used to convert the raw pixel values of multispectral images into reflectance during postprocessing (MicaSense, 2017b). The reflectance in each band of the CRP used in this study is shown in Table 2-1.

Table 2-1. Wavelength information of the camera and the reflectance in each band of the calibration reflectance panel (CRP).

\begin{tabular}{llll}
\hline Band Name & Center Wavelength (nm) & Bandwidth* (nm) & Reflectance (\%) \\
\hline Blue (B) & 475 & 20 & 49.2 \\
Green (G) & 560 & 20 & 49.3 \\
Red (R) & 668 & 10 & 49.1 \\
Red edge (Re) & 717 & 10 & 48.7 \\
Near-infrared (NIR) & 840 & 40 & 49.0 \\
\hline
\end{tabular}

* Bandwidth is defined as the full width at half maximum (FWHM).

The multispectral camera was mounted on a UAV DJI Matrice 600 Pro (DJI, Shenzhen, China) with the camera facing the ground vertically (nadir view). The UAV platform was controlled using a flight control App Autopilot (Hangar Technology, Austin, TX, USA) that allows setting up flight path, flying speed, and elevation prior to the flights. Images were taken at $30 \mathrm{~m}$ above ground level (AGL) with a ground sample distance (GSD) of $20.8 \mathrm{~mm} \cdot$ pixel $^{-1}$. The desired flight speed and the number of paths were carefully determined to obtain sufficient overlaps (forward overlap of $87.5 \%$ and side overlap of $84.5 \%$ in this case) to cover the whole field. The images were acquired from 13:15 to 15:00 CDT on Aug. 27, Sept. 14 and Sept. 27, 2018.

\subsubsection{Image processing}

The geo-referenced multispectral images were downloaded from the SD card and uploaded to a UAV image processing software Pix4D Mapper (Pix4D, Lausanne, Switzerland) to generate orthomosaic images of the field. After all the images were 
imported, the camera GNSS information was read automatically from the EXIF metadata. The images of the CRP were selected and the reflectance was inputted manually following an official instruction (MicaSense, 2018). The “Ag Multispectral processing” template was chosen for this processing. When the processing was completed, the orthomosaic image of each band was generated and exported as a .tif image, which was processed using the Image Processing Toolbox and Computer Vision System Toolbox of MATLAB (ver. 2016b, The MathWorks, Natick, MA, USA).

The background (soil and other non-crop) information was first removed from orthomosaic images using color thresholds. Figure 2-1 a and c are the colorful images with the red (R), green (G) and blue (B) channels of images acquired on Aug. 27 and Sept. 27 and they show different color contrasts between soil and soybeans at different growth stages. A blue normalized difference vegetation index (BNDVI) (Yang et al., 2004) that could signify the crop pixels and suppress non-crop pixels was used to remove the background of images on Aug. 27 and Sept. 14 as it had the highest contrast between soil and soybeans (Figure 2-1b). The blue-wide dynamic range vegetation index (BWDRVI) (Hancock \& Dougherty, 2007) was used for images on Sept. 27 as it was found to have higher contrast than BNDVI when soybean leaves started turning yellow and similar to soil color (Figure 2-1d). From the histograms in Figure 2-1 e and f, pixels with BNDVI values higher than 0 or with BWDRVI values higher than 0.7 were considered as soybean lines.

$$
\begin{gathered}
B N D V I=\frac{\text { nir }- \text { blue }}{\text { nir }+ \text { blue }} \\
B W D R V I=\frac{0.1 \times \text { nir }- \text { red }}{0.1 \times \text { nir }+ \text { red }}
\end{gathered}
$$



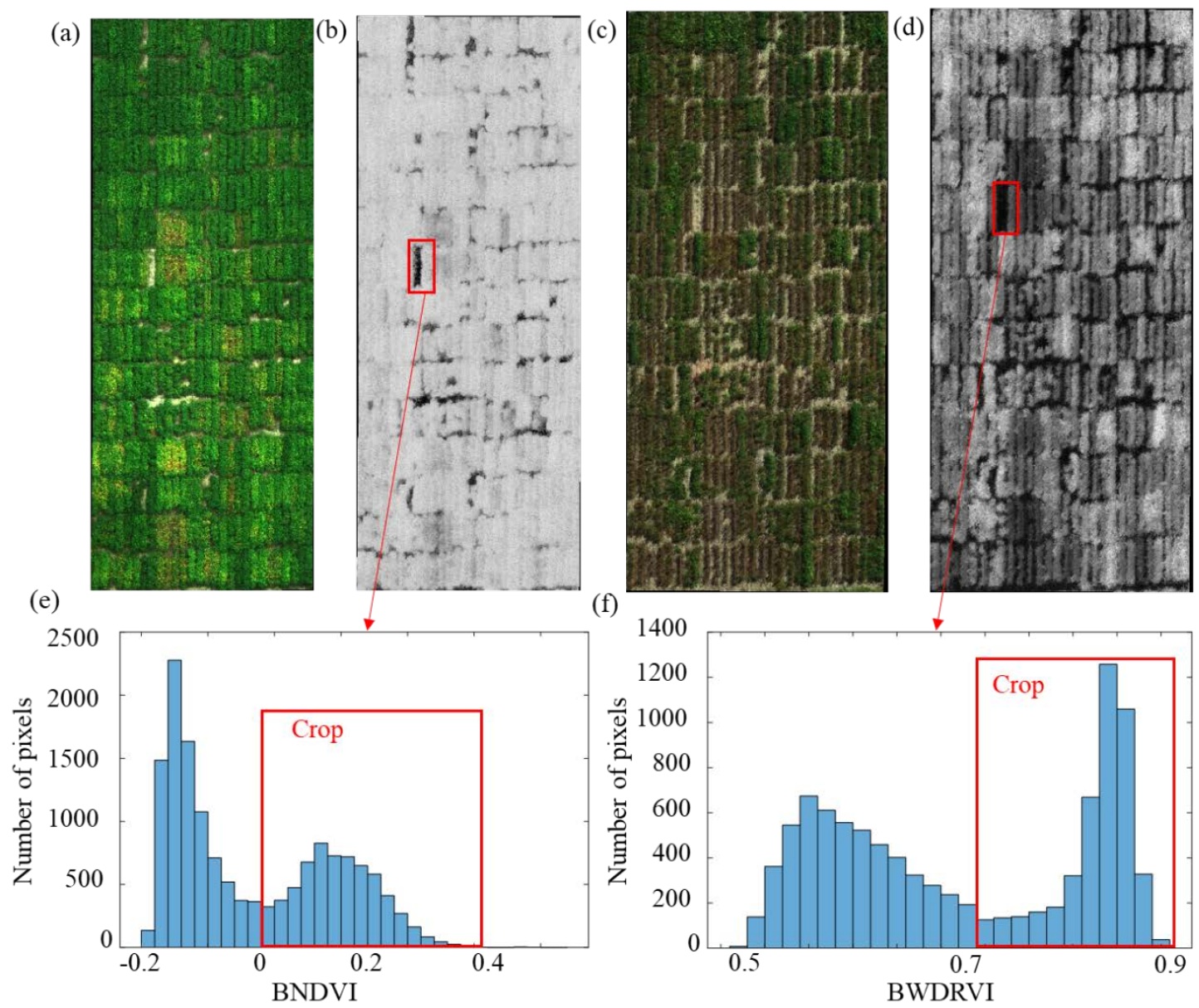

Figure 2-1. Orthomosaic images of part of the soybean field. (a) and (c) are the colorful images with the red (R), green (G) and blue (B) channels of images acquired on Aug. 27 and Sept. 27. (b)

The grayscale image of blue normalized difference vegetation index (BNDVI) derived from images taken on Aug. 27 and (d) the grayscale image of blue-wide dynamic range vegetation index (BWDRVI) derived from images taken on Sept. 27. (e) and (f) are histograms of image pixel values in the two red rectangles.

After background removal, individual soybean lines were segmented from one of the five-band images (the blue band images were used in this case) by manually drawing vertical and horizontal lines in alleys between plots. The segmented individual plots were saved as binary masks and then applied to the rest bands to get images of individual plots in each band. Each segmented plot has a unique plot number associated according to its physical position in the field and the number was saved with images of the plots. The 326 soybean samples were recognized by matching plot numbers in ground reference data with 
those in segmented images.

Fifty-four vegetation indices (VIs) were extracted from the five-band images using the formula summarized by Agapiou et al. (2012) and Henrich et al. (2009). The formula and a brief description of the selected VIs are included in Appendix Table A1. The mean and standard deviation (std) values of these VIs and the pixels of the five-band images of every single row were calculated as image features. In addition, we also calculated the mean and the std values of the hue, saturation (S) and value (V) in HSV color space and the $\mathrm{L}^{*}$, $\mathrm{a}^{*}$ and $\mathrm{b} *$ in CIELAB color space, which was converted from R, G, and B channels using MATLAB function 'rgb2hsv' and 'rgb2lab'. In total, 130 image features were extracted for further processing.

\subsubsection{Maturity date and adjusted maturity date}

The status of maturity was determined when approximately $95 \%$ of the pods in a line had achieved mature pod color by visual assessment (Fehr et al., 1971), ignoring the delayed leaf drop and green stems that might introduce variances in canopy images of soybean lines with the same maturity date. Additionally, maturity dates of soybean lines are usually determined visually by breeders in an interval of seven days and the dates of the breeding lines matured within the intervals were estimated based on experiences, therefore, the maturity dates may allow a one-or two-day error (Yu et al., 2016). Figure 22 shows images of three soybean lines taken on Sept. 27. Although recorded with the maturity date of Sept. 25, they had different numbers of green leaves remaining on the canopy. Line 7344 (left) had rare leaves remaining so that it could be derived from the images that its pods have turned to mature color. However, a certain number of green leaves on the canopy of line 6913 (right) covered most of its stems. The real scenarios could be 
that either its pods had reached the mature color but with delayed dropping leaves or its maturity date was estimated too early in the interval of seven days.
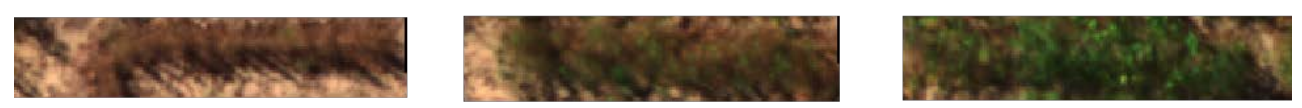

Figure 2-2. Illustration of variations in canopy images of the soybean lines matured on Sept. 25. Images were generated using $\mathrm{R}, \mathrm{G}$ and $\mathrm{B}$ bands from the multispectral camera. Breeding line no. from left to right is 7344, 7335 and 6913.

In order to tolerate the variances in canopy leaves, we used a new parameter adjusted maturity date (adMD), calculated as Eq. 2-3. The adMD calculates the variances in canopy images of the soybeans matured on the same day and applied the variances to the manually measured maturity date. The original maturity dates would be extended to a more precise format (22.2, 23.7 and 25.6 for soybean lines in Figure 2-2 from left to right) considering human error and canopy variances.

$$
\operatorname{adMD_{i}}=\frac{1}{n}\left(\sum_{1}^{n} \frac{x_{i n}-m_{i n}}{s t d_{i n}}\right)+M D_{i}
$$

where $a d M D_{i}$ is the adjusted maturity date of the $i^{\text {th }}$ soybean line. $i$ represents the $i^{\text {th }}$ soybean line, $i=1,2, \ldots, 326$ and $n$ represents the $n^{\text {th }}$ image feature used in an estimation model, $n$ $=1,2, \ldots, 130 . M D_{i}$ is the maturity date of the $i^{\text {th }}$ soybean line that is determined by breeders. $x_{i n}$ is the value of the $i^{\text {th }}$ soybean line in the $n^{\text {th }}$ image feature. $m_{i n}$ is the mean of all soybean lines matured on the same day with the $i^{\text {th }}$ soybean line in the $n^{\text {th }}$ image features. $s t d_{i n}$ is the standard deviation of all soybean lines matured on the same day with the $i^{\text {th }}$ soybean line in the $n^{\text {th }}$ image features.

\subsubsection{Data analysis}

All statistical analyses were conducted using the statistical toolboxes in MATLAB. To evaluate the potential of UAV-based imagery in estimating maturity dates, PLSR 
models were built using image features as predictors and maturity dates as responses for the data collected on three days. PLSR models create linear combinations (known as components) of the original predictor variables (image features) to explain the observed variability in the responses (measured maturity dates). Additionally, PLSR largely is able to reduce the variability and instability of estimated responses caused by multicollinearity among predictors (Ishtiaq \& Abdul-Aziz, 2015). The PLSR was conducted using the 'plsregress' function with 10 -fold cross-validation. The estimation accuracy was evaluated using the RMSE of the estimated responses.

To develop a parsimonious and interpretable PLSR model for each data set, variable importance in projection (VIP) scores were used to select predictors by estimating the importance of each predictor in a PLSR model. A predictor with a VIP score close to or greater than 1 can be considered important in a given model (Eigenvector Research, 2018). The VIP scores of each predictor were calculated using MATLAB codes suggested by the MathWorks Support Team (2019).

The variance inflation factor (VIF) was also calculated to remove image features with high collinearity (James et al., 2013). The VIF is calculated as a dependent variable of the coefficient of determination $\left(R^{2}\right)$ of an individual feature against all other features. $R^{2}$ was obtained by calling the parameter of 'Im.Rsquare.Ordinary' in the 'fitlm' function. This step was performed in a loop that the variable with the highest VIF was removed each time and the loop would not stop until the highest VIF is less or equal to 5 (Chen et al., 2014). The higher the VIF value an image features have, the higher the collinearity is between this feature with others.

To better interpret the PLSR models, the correlation between each selected 
predictor and maturity dates was calculated using 'corr' function with the 'Pearson' option. As the multispectral images were collected on the three days at different growth stages of soybean lines, the changes in image features between every two days may indicate the transitions from the immature to mature status of soybean lines. The changing rate between each two data collection days was calculated using Eq. 2-4.

$$
\text { Changing rate }(i)=\frac{P(i+1)-P(i)}{P(i)}
$$

where $P$ is each selected predictor of single soybean lines on the $i=1^{\text {st }}, 2^{\text {nd }}$ and $3^{\text {rd }}$ time of data collection.

\subsection{Results}

\subsubsection{Estimation of soybean maturity dates using PLSR}

The maturity dates estimated using the PLSR models with 10-fold cross-validation on the three days are shown in Figure 2-3. Figure 2-3 a illustrates the percent variance explained in maturity dates with the number of PLSR components. Variance in maturity dates can be explained up to 59\%, 77\% and $83 \%$ using image features on Aug. 27, Sept. 14 and Sept. 27, respectively. Figure 2-3 b shows the changes of RMSE with the number of PLSR components and the optimal number of components for each day was determined when the RMSE reaches the minimum on that day. The estimation using image features on Sept. 27 had the lowest RMSE of 1.7 days with five components followed by the one on Sept. 14 (RMSE $=1.8$ days) with 24 components and Aug. $27(R M S E=2.7$ days $)$ with seven components. Thus, the optimal numbers of components for the PLSR model on the three days are 7, 24 and 5, respectively.

Figure 2-3 c and e show the correlations between the manual maturity dates and 
predicted maturity dates from PLSR models with the optimal numbers of components. When using image features of soybean lines in the middle of their maturity stage (R8, Sept. 27), there was the best agreement $\left(R^{2}=0.82\right)$ of predicted maturity dates and manual measurements.
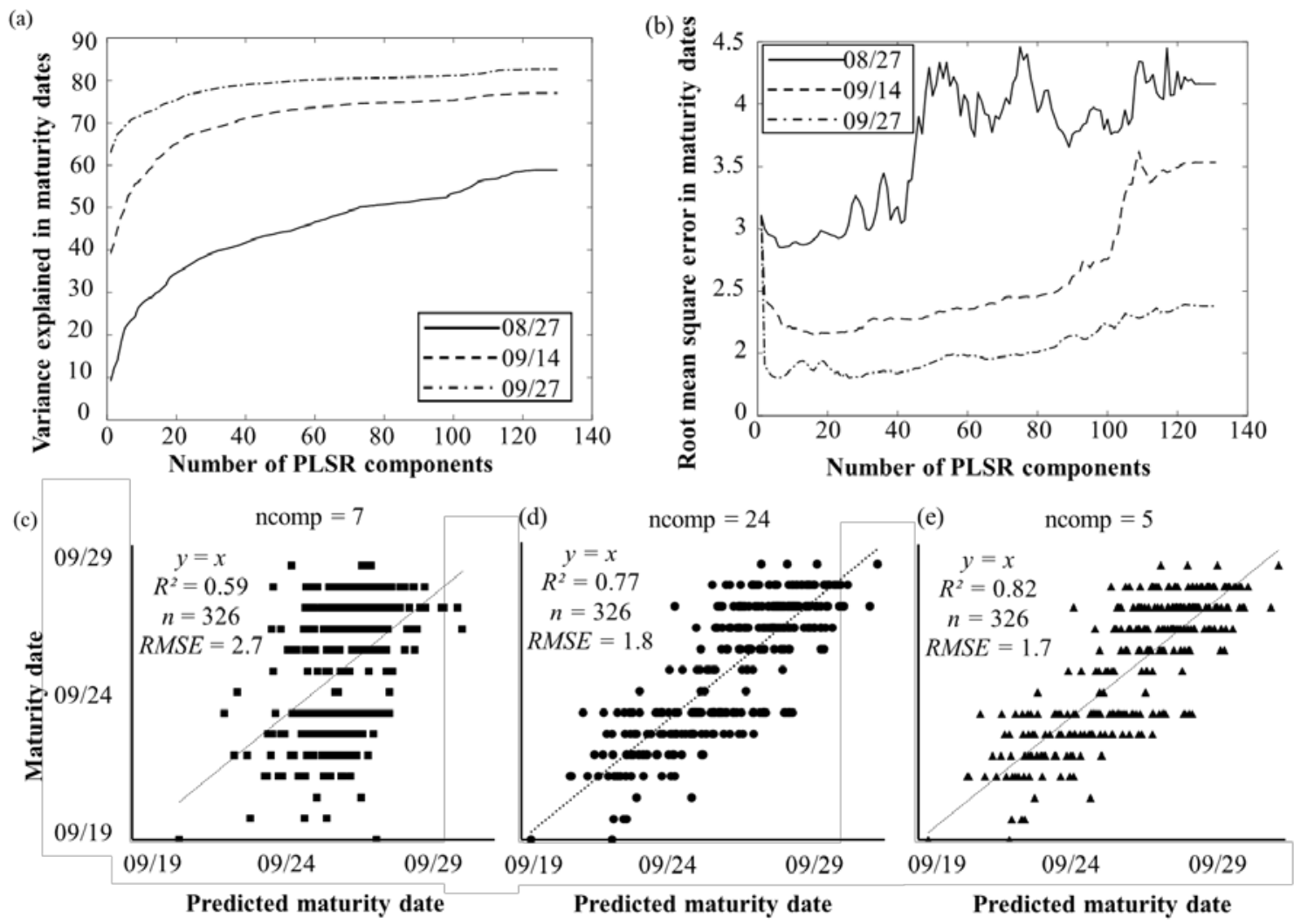

Figure 2-3. Estimation of maturity dates using the PLSR model. (a) Percent of variance explained in the maturity dates as a function of the number of components in the PLSR model. (b) Mean square error in the maturity dates as a function of the number of components. (c)-(e) The correlations between manual maturity dates and predicted maturity dates using images features taken on Aug. 27, Sept. 14 and Sept. 27, respectively. ncomp represents the optimal number of components.

\subsubsection{Model parsimony}

Figure 2-4 shows the VIP scores of 130 predictors of the PLSR models on the three days. There were 42, 56 and 73 predictors with VIP scores equal or greater than 1 in models on August 27, Sept. 14 and Sept. 27, respectively. VIFs of these predictors were then 
calculated and those with VIFs less than 5 are shown in Table 2-2. Table 2-2 also shows the Pearson correlations between the maturity dates and the selected predictors (image features) from PLSR models on the three days. It can also be seen that few image features were overlapping in the PLSR models for all three days. The possible reason is that strong collinearities were existing among all the 130 image features so that those with high Pearson correlations were not selected due to their high VIFs ( $>5)$.

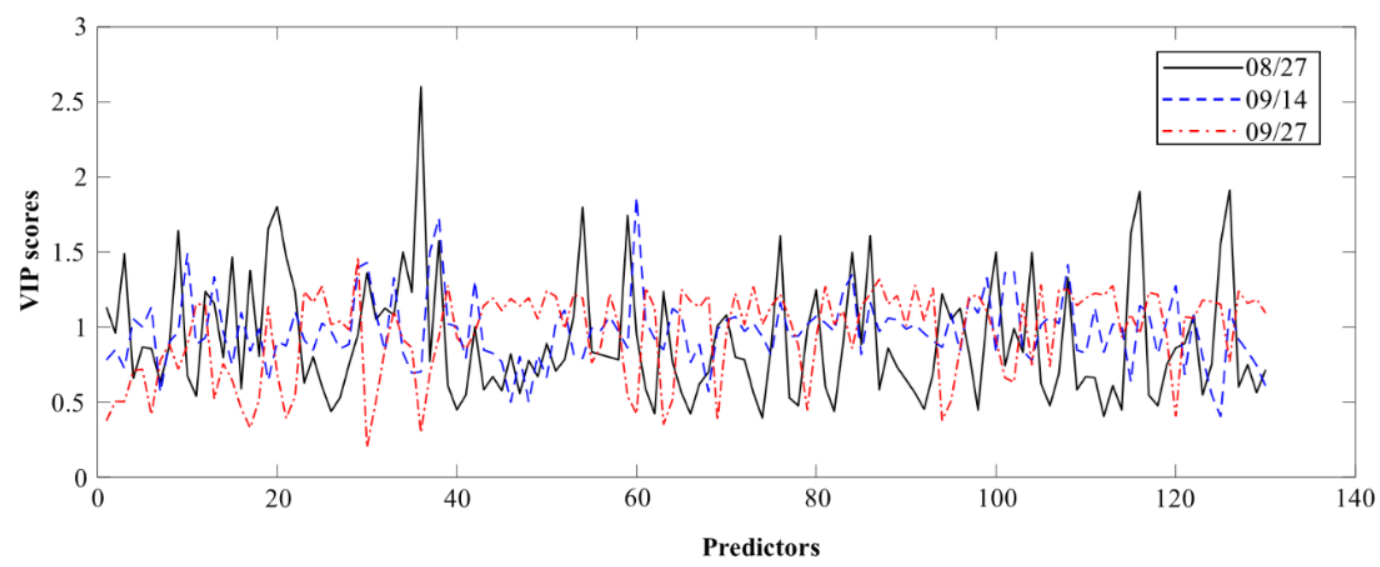

Figure 2-4. Variable importance in projection (VIP) scores of 130 predictors of the PLSR models on the three days.

Table 2-2. Pearson correlations between the predictors with VIP scores $\geq 1$ and variance inflation factors (VIFs) $<5$ and maturity dates on the three days.

\begin{tabular}{llll}
\hline Image features & Aug. 27 & Sept. 14 & Sept. 27 \\
\hline re_mean & $-0.596^{*}$ & - & - \\
S_mean & -0.501 & $-0.697^{* *}$ & - \\
CCCI_std & -0.487 & $-0.930^{* * *}$ & - \\
CIrededge_mean & 0.504 & - & 0.368 \\
CVI_std & -0.389 & - & - \\
CI_std & 0.184 & $-0.662^{* *}$ & - \\
GDVI_std & 0.177 & $0.861^{* * *}$ & - \\
H_mean $\dagger$ & 0.102 & - & - \\
NormG_std & 0.413 & $-0.831^{* * *}$ & - \\
IF_std & -0.170 & - & - \\
RRI2_std & -0.239 & -0.227 & - \\
CI_mean & - & $-0.935^{* * *}$ & - \\
H_std & - & $-0.915^{* * *}$ & - \\
GRVI_mean & - & $0.922^{* * *}$ & - \\
MTVI2_std & - & $0.795^{* * *}$ & - \\
hue_std $\ddagger$ & - & - & $0.959^{* * *}$
\end{tabular}




\begin{tabular}{llll} 
GEMI_mean & - & - & $0.977 * * *$ \\
GRNDVI_std & - & - & $0.959 * * *$ \\
BNDVI_mean & - & - & $0.934^{* * *}$ \\
IF_mean & - & - & $-0.991^{* * *}$ \\
\hline
\end{tabular}

$*$, ** and $* * *$ indicate the significance at $p=0.05,0.01$ and 0.001 levels, respectively. $\dagger$ and $\ddagger$ represent the image feature hue in HSV color space but was calculated using different methods. $\dagger$ uses the equation in Appendix Table A1. $¥$ is converted from the RGB image using the ' $r g b 2 h s v$ ' function in MATLAB.

In order to understand the different growth trends of soybean lines matured sequentially, the Pearson correlations between maturity dates and the selected image features in Table 2-2 were calculated and shown in Table 2-3. It can be seen that only three image features re_mean, CI_mean and IF_mean had significant linear relationships with the maturity dates on Aug. 27, while the majority of image features on Sept. 14 and Sept. 27 are of significance.

Table 2-3. Pearson correlations between maturity dates and the means of image features at each maturity date on the three days.

\begin{tabular}{llll}
\hline Image features & Aug. 27 & Sept. 14 & Sept. 27 \\
\hline re_mean & $-0.596^{*}$ & -0.414 & $0.890^{* * *}$ \\
S_mean & -0.501 & $-0.697^{* *}$ & $-0.830^{* * *}$ \\
CCCI_std & -0.487 & $-0.930^{* * *}$ & $0.605^{*}$ \\
CIrededge_mean & 0.504 & $0.928^{* * *}$ & 0.368 \\
CVI_std & -0.389 & $-0.718^{* *}$ & $-0.552^{*}$ \\
CI_std & 0.184 & $-0.662^{* *}$ & $0.931^{* * *}$ \\
GDVI_std & 0.177 & $0.861^{* * *}$ & $0.845^{* * *}$ \\
H_mean & 0.102 & $-0.830^{* * *}$ & -0.409 \\
NormG_std & 0.413 & $-0.831^{* * *}$ & $0.916^{* * *}$ \\
IF_std & -0.170 & $-0.921^{* * *}$ & $-0.585^{*}$ \\
RRI2_std & -0.239 & -0.227 & $0.981^{* * *}$ \\
CI_mean & $-0.595^{*}$ & $-0.935^{* * *}$ & $-0.886^{* * *}$ \\
H_std & -0.490 & $-0.915^{* * *}$ & 0.268 \\
GRVI_mean & 0.497 & $0.922^{* * *}$ & 0.499 \\
MTVI2_std & -0.035 & $0.795^{* * *}$ & $0.989^{* * *}$ \\
hue_std & -0.413 & $-0.931^{* * *}$ & $0.959^{* * *}$ \\
GEMI_mean & -0.004 & $0.857^{* * *}$ & $0.977 * * *$ \\
GRNDVI_std & 0.242 & $-0.889^{* * *}$ & $0.959^{* * *}$ \\
BNDVI_mean & 0.356 & $0.879^{* * *}$ & $0.934^{* * *}$ \\
IF_mean & $-0.544 *$ & $-0.879^{* * *}$ & $-0.991^{* * *}$ \\
\hline Indicate the significance at $p=0.05,0.01$ and 0.001 levels, respectively.
\end{tabular}

Figure 2-5 shows the predicted maturity dates using three PLSR models with 20 selected image features and their changing rates. The estimations were performed using the 
same method mentioned in section 2.5. The optimal numbers of components for the three PLSR models were 13, 5 and 4, respectively. Compared with Figure 2-3 d and e, the PLSR models with changing rates in the selected image features taken between Sept. 14 and Sept. 27 improved the estimation accuracy (Figure 2-5 b and c). The results indicate that there are certain patterns that image features of soybean lines with different maturity dates changed over time and these patterns could help to recognize the maturity dates. From Figure 2-5 a and b, the model with the changing rates between Sept. 14 and Sept. 27 had higher estimation accuracy than the one with the changing rates between Aug. 27 and Sept.

14 , showing the challenges in estimating maturity dates at early stages.

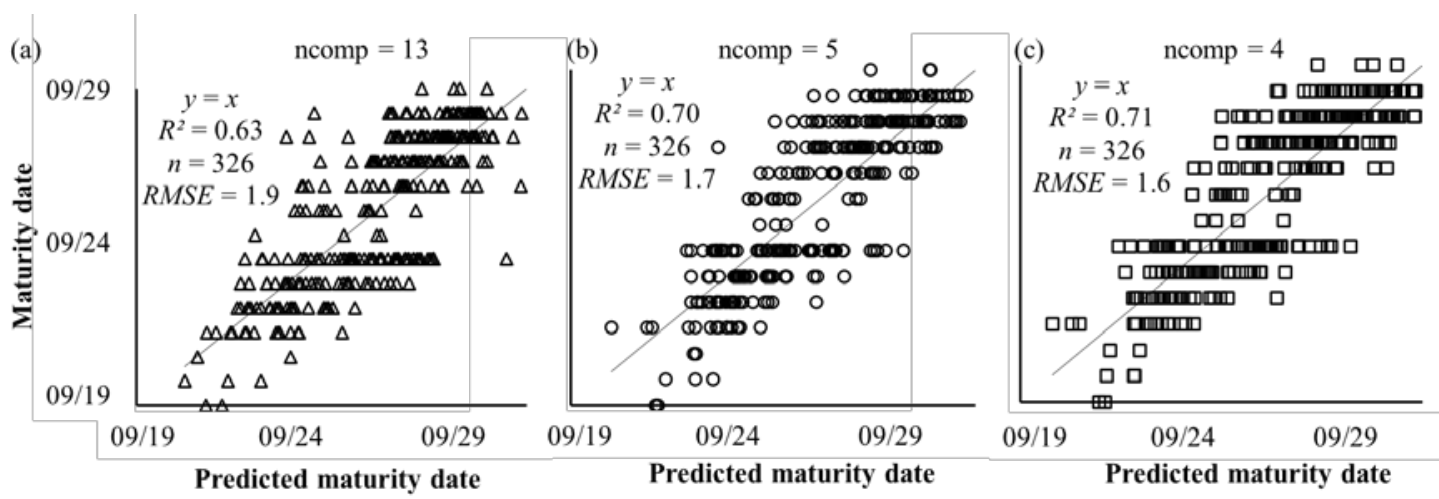

Figure 2-5. Estimation of maturity dates using PLSR models with the selected image features and the changing rates. (a) The correlations between manual maturity dates and predicted maturity dates using the selected images features taken on Sept. 14 and their changing rates between Aug. 27 and Sept. 14. (b) The correlations using the selected images features taken on Sept. 14 and their changing rates between Sept. 14 and 27. (c) The correlations using the selected images features taken on Sept. 27 and their changing rates between Sept. 14 and 27.

\subsubsection{Adjusted maturity dates based on the variances in image features}

Even though we can observe the monotonically increasing and decreasing trends in the selected image features against the maturity dates (Table 2-2), the variances in some days overlapped with other days (Figure 2-6 a and b), especially when those soybean lines were close to their maturity, for example, the large variances in NDVIrededge_mean taken on Sept. 14 were observed in those lines matured on Sept. 19 and 20, and the large variances 
in CCCI_mean taken on Sept. 27 were observed from Sept. 21 to 27.

Figure 2-6 c shows canopy images in these two vegetation indices of soybean lines (as shown in Figure 2-1) that were determined by visual ratings maturing on the same day (Sept. 25). It can be seen that the dry stems had the highest CCCI values, while the green leaves had the lowest. When soybean lines had reached their R7 stages, the water content of the seeds is less than $50 \%$ and when they at the R8 stages, the water content is less than 30\% (Fehr et al., 1971). As an indicator of water stress suggested by Barnes et al. (2000), CCCI could represent various water content in soybean lines.

The NDVIrededge was first proposed by Gitelson and Merzlyak (1994) and found that it was highly proportional to crop leaf chlorophyll content. It was calculated using the $\mathrm{R}(668 \mathrm{~nm})$ band with the Re $(717 \mathrm{~nm})$ band and has shown a strong linear proxy $\left(R^{2}=\right.$ 0.94) of the green portion of the fraction of absorbed photosynthetically active radiation (fAPAR) that is sensitive to chlorophyll content in maize and soybean canopy (Viña \& Gitelson, 2005). It is consistent with our observation that the soybean line with more remaining leaves (No. 6913) had higher NDVIrededge values while the dry lines (e.g., No. 7344) had very low values. 


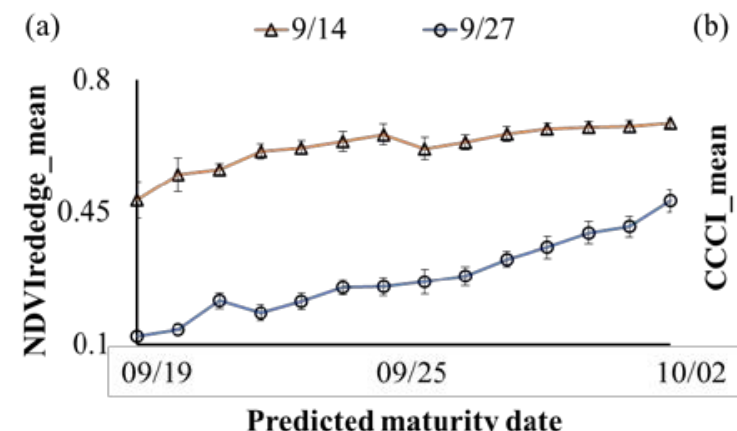

Predicted maturity date

(b)

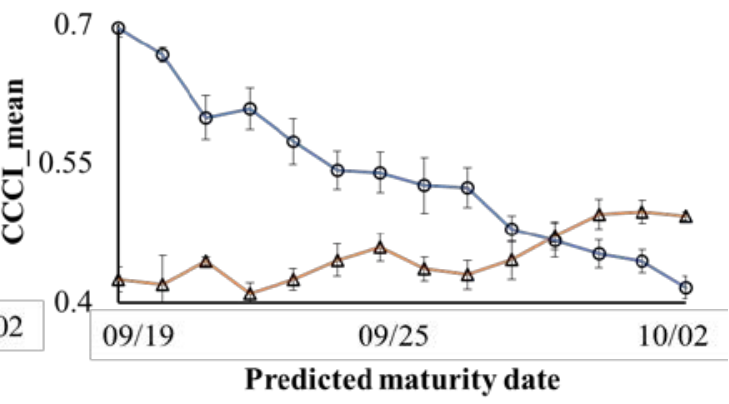

(c)

$$
\text { RGB }
$$

No. 7344

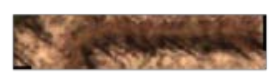

No. 7335

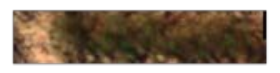

No. 6913

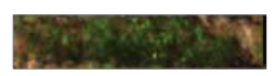

NDVIrededge

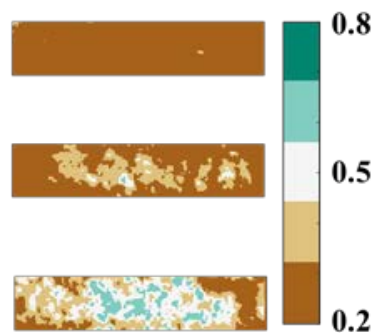

Figure 2-6. Vegetation indices CCCI and NDVIrededge. (a)-(b) The relationships between maturity dates and NDVIrededge_mean and CCCI_mean collected on Sept. 14 and 27. (c) Canopy images of the soybean lines matured on Sept. 25 in RGB, CCCI and NDVIrededge.

The maturity dates of soybean lines were adjusted based on the variances of NDVIrededge_mean and CCCI_mean taken on Sept. 14 and 27. The relationships between maturity dates and the adjusted maturity dates were shown in Figure 2-7. The PLSR models were used to predict the maturity dates using the same method mentioned in section 2.5 and the estimations are shown in Figure 2-7 b and c. 

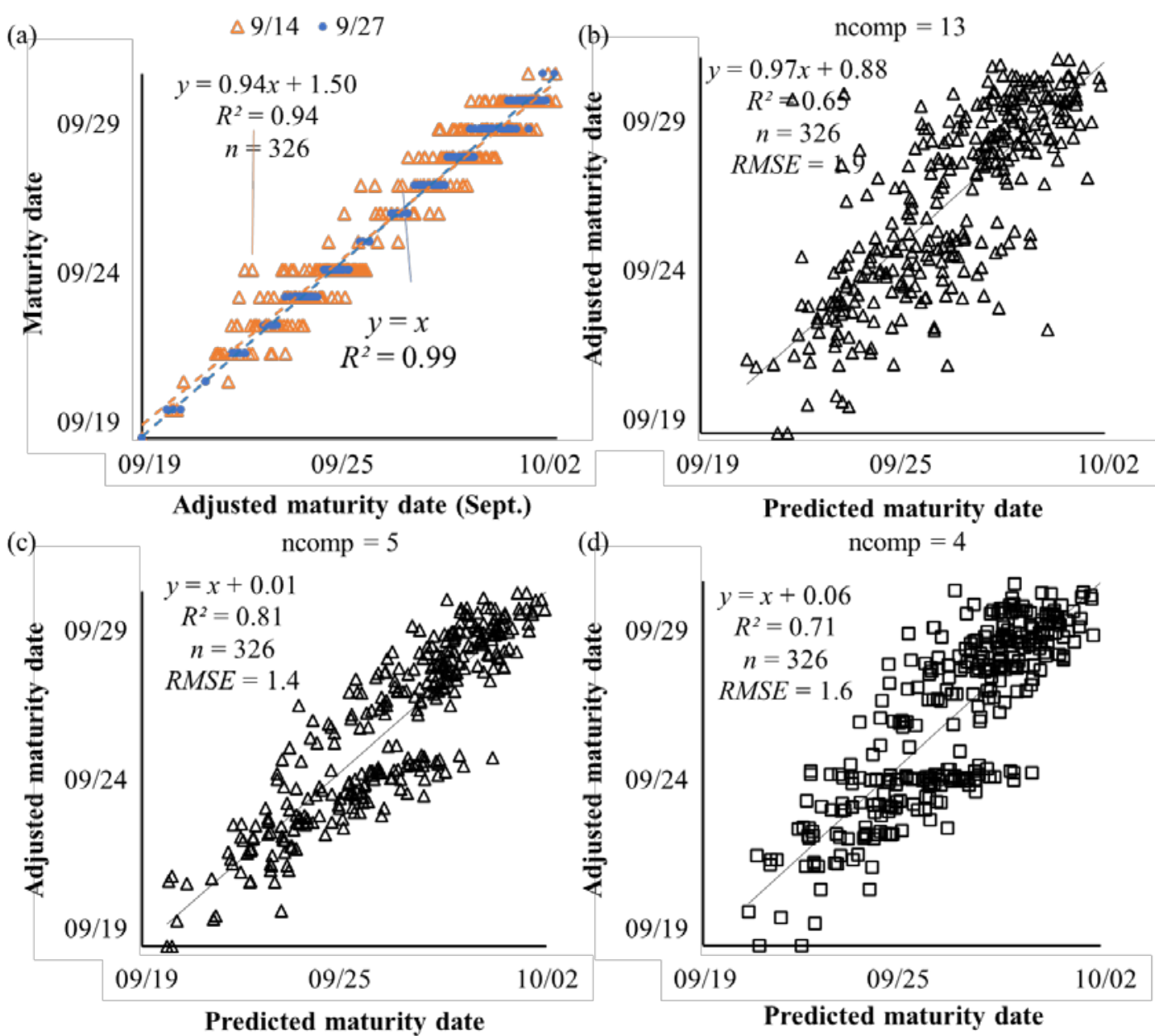

Figure 2-7. Adjusted maturity dates. (a) The relationship between maturity dates and the adjusted maturity dates based on the variances of NDVIrededge_mean and CCCI_mean taken on Sept. 14 and 27. (b) The correlations between adjusted maturity dates and predicted maturity dates using the selected image features taken on Sept. 14 and their changing rates between Aug. 27 and Sept. 14 (c) The correlations using the selected image features taken on Sept. 14 and their changing rates between Sept. 14 and 27. (d) The correlations using the selected image features taken on Sept. 27 and their changing rates between Sept. 14 and 27.

\subsection{Discussion and future work}

\subsubsection{Estimation of soybean maturity dates at different growth stages}

The estimation of soybean maturity dates using the PLSR model with image features collected on different days in Figure 2-2 shows that the best agreement of predicted maturity dates and manual measurements was made by image features in the middle of 
their maturity stage, while the worst is by those collected when none of the soybean lines started maturing.

From Table 2-2, only one image feature (re_mean) had a significant linear relationship with the maturity dates on Aug. 27, while there were nine and five image features highly correlated ( $p$-value $<0.05$ ) with the maturity dates for Sept. 14 and 27, respectively, indicating the challenge in estimating maturity date at the early stages. We can also see that there were six common image features selected for PLSR models on Aug. 27 and Sept. 14, and five of them had significant linear relationships on Sept. 14. These image features had the potential to be used as predictors, but they have not shown the trends at the early stage when mostly soybean lines remained green (Figure 2-1a).

Again, it can be seen from Table 2-3 that only three image features re_mean, CI_mean and IF_mean had significant linear relationships with the maturity dates on Aug. 27, while the majority of image features on Sept. 14 and 27 were of significance. It may indicate a higher accuracy in estimation of maturity date using data from later stages than early stages, as implied by the estimations from the PLSR models (Figure 2-3).

\subsubsection{Selected features for the parsimonious models}

For all three days, five image features (CIrededge_mean, GDVI_std, GRVI_mean and BNDVI_mean) had positive linear relationships with the maturity dates, and four image features (S_mean, CVI_std, IF_std, CI_mean and IF_mean) were negatively related to the maturity dates, suggesting that these image features of all soybean lines kept increasing or decreasing from the beginning to the full maturity stage so that they can maintain consistent trends no matter which stages the image features were collected at. As shown in Figure 2-8a, for all the soybean lines, the changing rates in CI_mean were above 
0 due to the increased CI_mean in all soybean lines from Sept. 14 to 27. It was also observed that the changing rates in soybean lines that matured later were greater than those in lines that matured early, leading to a positive linear relationship between the changing rates in CI_mean and the maturity dates.

It should be noticed that the Pearson correlation coefficients of the other 11 image features in Table 2-3 had opposite signs for the three days, especially for the latter two days, such as re_mean, CCCI_std, CI_std, NormG_std, hue_std and GRNDVI_std. The reason may be that for the soybean lines matured on different days, there were different changing rates in such features. From Figure 2-8a, the changing rates in hue_std were significantly less than 0 for lines matured before Sept. 24, indicating the hue_std of these lines decreased from Sept. 14 to 27. For lines matured during Sept. 24 to 27, the changing rates in hue_std were very close to 0, suggesting that the hue_std in these lines barely changed between two data collection days. For lines matured after Sept. 27, the changing rates were significantly greater than 0 , showing that there were increased hue_std in these lines. It might be due to the gradual occurrence of yellow leaves when the soybean lines were closed to their maturity (Figure 2-8b), leading to high variances in hue, while those lines that matured late still had more green leaves (Figure 2-8c). When hue was calculated on Sept. 27, matured soybean lines had more dry leaves than fresh leaves (Figure 2-8d), leading to low variances, while the late-matured lines were stepping into their R8 stages (Figure 2-8e), resulting in high variances. Similar situations could happen in other image features with opposite linear relationships for the latter two days. 


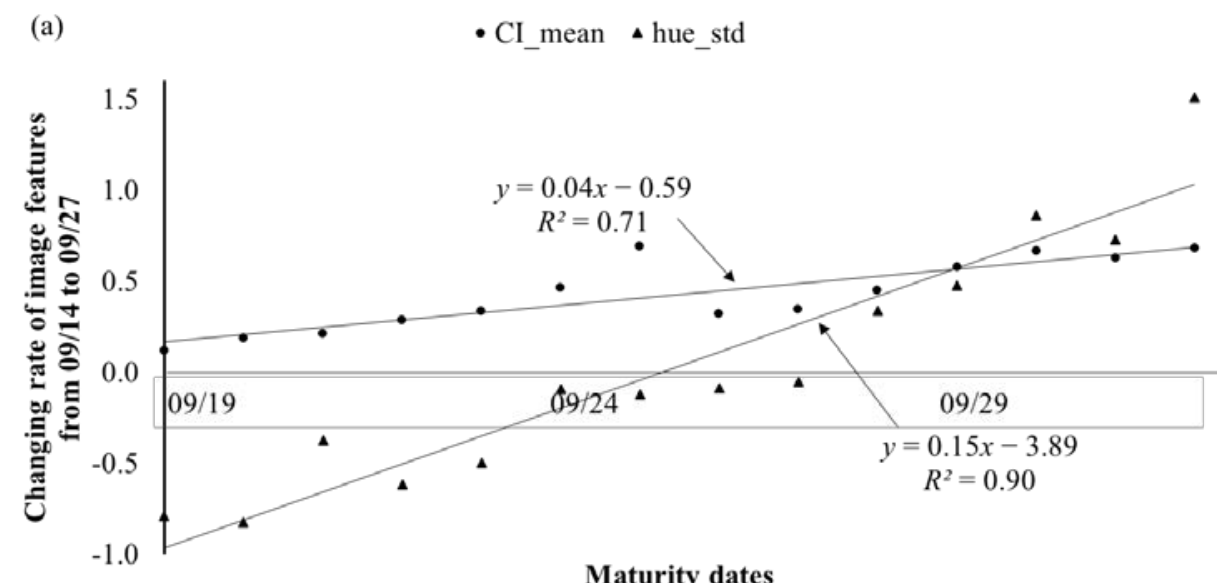

(b)

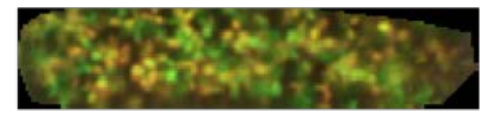

(c)

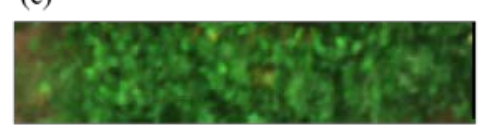

(d)

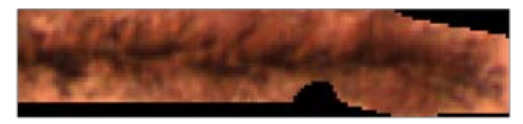

(e)

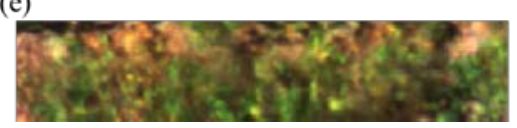

Figure 2-8. The changing rates in CI_mean and hue_std from Sept. 14 and 27. (a) The changing rates in CI_mean and hue_std. (b) and (d) are RGB images of a line matured on Sept. 19, and they were taken on Sept. 14 and 27, respectively. (c) and (e) are RGB images of a line matured on Oct. 1, and they were taken on Sept.14 and 27, respectively.

\subsubsection{Adjusted maturity dates}

In Figure 2-7a, the adjusted maturity dates for both image collection days were extended around their observed maturity dates, which might help to tolerate the variances of canopy green leaves as well as errors from subjective judgments and estimations of breeders. By using the adjusted maturity dates as a ground reference, the RMSE dropped from 1.7 to 1.4 and the $R^{2}$ increased to 0.81 using the selected images features taken on Sept. 14 and their changing rates between Sept. 14 and 27, while nearly no changes occurred on the other two models. It might be because these two image features captured more variances on Sept. 14 than those on Sept. 27.

By personal communication with breeders, RMSE within 1.5 days can be considered as a tolerable error in soybean breeding programs. Compared to previous 
studies, our study shows improvements in predicting the exact maturity dates instead of the classification between mature and immature lines (Yu et al., 2016), as well as making a more practical prediction (with an acceptable accuracy) for breeding programs (Christenson et al., 2016). Therefore, it can be concluded that it is a promising method to predict soybean maturity dates using UAV-based multispectral image features by screening the soybean lines once at the beginning of maturity stage to quantify the variances in canopies and another one at the middle of the full maturity stage to track the canopy changes.

As the adjusted maturity dates are first proposed in this study to tolerate the variances of canopy green leaves for the purpose of predicting soybean maturity dates using canopy image features, the maturity dates were adjusted based on the variances in two image features with known matured dates in a single environment. In soybean breeding programs, RMs of a variety from around 40-50 environments are required to assign an MG to the variety. Therefore, the variances need to be quantified in future studies by repeating the experiments in multiple environments and documenting the image features.

Errors could be introduced by many factors regarding image features. Due to the limited field of view of the multispectral camera, the 3.64 ha field was fully screened by three flight missions during the time period of more than $2 \mathrm{~h}$, including taking off and landing the UAV, changing batteries and calibration. Light changes during image collection were conjunctions of many factors, such as the changing relative position between sun and drone, various light diffusions caused by the thin cloud in the sky and the random exiting thick clouds, which resulted in cloud shadows in the images (not observed in this study) (MicaSense, 2017a). Another inevitable source of variation is some blurred 
portions of orthomosaic caused by image stitching. At the later stage of soybean growth, soybean lines with big canopies can touch and overlap with their neighbors and leaves on immature lines are yellow or in the transition to yellow, which were hard to be distinguished with soil. Under this scenario, blurs and even undetectable misalignments of breeding lines might occur in the orthomosaic.

\subsubsection{Future work}

In future studies, the variances in image features to adjust the maturity dates need to be quantified and derived by repeating the experiments in multiple environments and documenting image features. There were also two major issues expected to be addressed to reduce errors introduced during the image collection and processing pipeline. First, as an image pixel value is the reflectance of incoming solar irradiance, the variation in light conditions could introduce variations to image-derived features. The relationship between light changes during UAV flight missions and camera responses (reflectance) should be further explored and flight dynamics (drone pose, sun's position, light diffusion, clouds, etc.) need to be better integrated to describe the light changes. Second, variations are inevitably introduced when images are stitched based on feature recognition. Instead of localizing individual breeding lines from an orthomosaic, efforts have to be made to investigate the potential of direct georeferencing that the geometric position of a breeding line is the conjunction of the relative position of the line in an image and the UAV position where the image was taken. With direct georeferencing, flight attitude and speed can be liberated from high image overlaps ( $\geq 70 \%$ for creating orthomosaic images) so that the time issue and light variation can be moderated consequently. 


\subsection{Conclusions}

The potential of predicting maturity dates of soybean breeding lines using UAVbased multispectral imagery was investigated in this paper. Maturity dates of 326 soybean breeding lines were taken using visual ratings from the beginning maturity stage (R7) to full maturity stage (R8), and the aerial multispectral images were also taken during this period on Aug. 27, Sept. 14 and Sept. 27. One hundred and thirty features were extracted from the five-band multispectral images. The maturity dates of soybean lines were predicted and evaluated using PLSR models with 10-fold cross-validation. The results showed that the estimations at later stages had better accuracy than those at earlier stages. Twenty image features with importance were then selected to simplify the PLSR models. The changing rates in image features between each of the two collection days were calculated. The estimation accuracy was improved by the simplified PLSR models with the selected image features and their changing rates. The adjusted maturity dates were proposed to tolerate the variances of canopy green leaves and calculated based on the variances in NDVIrededge_mean and CCCI_mean. The best prediction $\left(R^{2}=0.81, R M S E\right.$ = 1.4 days) was made by the PLSR model using selected image features taken on Sept. 14 and their changing rates between Sept. 14 and 27 as predictors and the adjusted maturity dates as responses with five components. This study outperformed other similar studies in terms of prediction accuracy and practical usefulness. Another contribution of this paper is that based on our observations from canopy images, the adjusted maturity dates were proposed to tolerate the variances of canopy green leaves as well as errors from subjective judgments and estimations of breeders. 


\section{References}

Agapiou, A., Hadjimitsis, D. G., \& Alexakis, D. D. (2012). Evaluation of Broadband and Narrowband Vegetation Indices for the Identification of Archaeological Crop Marks. Remote Sensing, 4(12), 3892

Barnes, E., Clarke, T., Richards, S., Colaizzi, P., Haberland, J., Kostrzewski, M., et al. (2000). Coincident detection of crop water stress, nitrogen status and canopy density using ground based multispectral data. Paper presented at the Proceedings of the Fifth International Conference on Precision Agriculture, Bloomington, MN, USA

Breene, K. (2018). Food security and why it matters. Retrieved from https://www.weforum.org/agenda/2016/01/food-security-and-why-it-matters/

Breseghello, F., \& Coelho, A. S. G. (2013). Traditional and Modern Plant Breeding Methods with Examples in Rice (Oryza sativa L.). Journal of Agricultural and Food Chemistry, 61(35), 8277-8286. 10.1021/jf305531j

Chen, D., Neumann, K., Friedel, S., Kilian, B., Chen, M., Altmann, T., et al. (2014). Dissecting the phenotypic components of crop plant growth and drought responses based on high-throughput image analysis. Plant Cell, 26(12), 46364655. 10.1105/tpc.114.129601

Christenson, B. S., Schapaugh, W. T., An, N., Price, K. P., Prasad, V., \& Fritz, A. K. (2016). Predicting Soybean Relative Maturity and Seed Yield Using Canopy Reflectance. Crop Science, 56(2), 625-643. 10.2135/cropsci2015.04.0237

Eigenvector Research, I. (2018). Vip. Retrieved from http://wiki.eigenvector.com/index.php?title=Vip

Fehr, W., Caviness, C. E., Burmood, D. T., \& Pennington, J. S. (1971). Stage of Development Descriptions for Soybeans, Glycine Max (L.) Merrill1. Crop Science, 11(6), 929-931. 10.2135/cropsci1971.0011183X001100060051x

Fehr, W. R. (1991). Principles of cultivar development: theory and technique. New York, NY: Macmillian. 
Gitelson, A., \& Merzlyak, M. N. (1994). Spectral Reflectance Changes Associated with Autumn Senescence of Aesculus hippocastanum L. and Acer platanoides L. Leaves. Spectral Features and Relation to Chlorophyll Estimation. Journal of Plant Physiology, 143(3), 286-292. https://doi.org/10.1016/S01761617(11)81633-0

Gitelson, A., Vina, A., Arkebauer, T. J., Rundquist, D. C., Keydan, G., \& Leavitt, B. (2003). Remote estimation of leaf area index and green leaf biomass in maize canopies. Geophysical Research Letters, 30(5), 52-51

Hancock, D. W., \& Dougherty, C. T. (2007). Relationships between Blue- and Red-based Vegetation Indices and Leaf Area and Yield of Alfalfa. Crop Science, 47(6), 2547-2556. 10.2135/cropsci2007.01.0031

Henrich, V., Götze, E., Jung, A., Sandow, C., Thürkow, D., \& Gläßer, C. (2009). Development of an Online indices-database: motivation, concept and implementation. EARSeL proceedings, EARSeL, Tel Aviv

Hincks, J. (2018). The World Is Headed for a Food Security Crisis. Here's How We Can Avert It. Retrieved from https://www.un.org/development/desa/en/news/population/world-populationprospects-2017.html

Ishtiaq, K. S., \& Abdul-Aziz, O. I. (2015). Relative Linkages of Canopy-Level CO2 Fluxes with the Climatic and Environmental Variables for US Deciduous Forests. Environmental Management, 55(4), 943-960. 10.1007/s00267-014-0437-1

James, G., Witten, D., Hastie, T., \& Tibshirani, R. (2013). An introduction to statistical learning (Vol. 112): Springer.

Masuka, B., Atlin, G. N., Olsen, M., Magorokosho, C., Labuschagne, M., Crossa, J., et al. (2017). Gains in Maize Genetic Improvement in Eastern and Southern Africa: I. CIMMYT Hybrid Breeding Pipeline. Crop Science, 57(1), 168-179. 10.2135/cropsci2016.05.0343

MathWorks Support Team. (2019). How to calculate the Variable Importance in Projection from outputs of PLSREGRESS. 
MicaSense. (2017a). DLS Sensor Basic Usage. Retrieved from

https://micasense.github.io/imageprocessing/MicaSense\%20Image\%20Processing \%20Tutorial\%203.html

MicaSense. (2017b). Use of Calibrated Reflectance Panels For RedEdge Data.

Retrieved from https://support.micasense.com/hc/en-us/articles/115000765514Use-of-Calibrated-Reflectance-Panels-For-RedEdge-Data

MicaSense. (2018). How to Process RedEdge Data in Pix4D. Retrieved from https://support.micasense.com/hc/en-us/articles/115000831714-How-to-ProcessRedEdge-Data-in-Pix4D

Mourtzinis, S., \& Conley, S. P. (2017). Delineating Soybean Maturity Groups across the United States. Agronomy Journal, 109, 1397-1403. 10.2134/agronj2016.10.0581

Peske, S. T., Höfs, A., \& Hamer, E. (2004). Seed moisture range in a soybean plant. Revista Brasileira de Sementes, 26, 120-124

Staton, M. (2017). What is the relationship between soybean maturity group and yield? Retrieved from https://www.canr.msu.edu/news/what_is_the_relationship_between_soybean_mat urity_group_and_yield

Sun, M. (2014). Efficiency study of testing and selection in progeny-row yield trials and multiple-environment yield trials in soybean breeding. (Doctor of Philosophy), Iowa State University, Ames, Iowa. Retrieved from https://lib.dr.iastate.edu/etd/13969

Viña, A., \& Gitelson, A. A. (2005). New developments in the remote estimation of the fraction of absorbed photosynthetically active radiation in crops. Geophysical Research Letters, 32(17). https://doi.org/10.1029/2005GL023647

Yang, C., Everitt, J. H., Bradford, J. M., \& Murden, D. (2004). Airborne hyperspectral imagery and yield monitor data for mapping cotton yield variability. Precision Agriculture, 5(5), 445-461

Yu, N., Li, L., Schmitz, N., Tian, L. F., Greenberg, J. A., \& Diers, B. W. (2016). Development of methods to improve soybean yield estimation and predict plant 
maturity with an unmanned aerial vehicle based platform. Remote Sensing of Environment, 187, 91-101. https://doi.org/10.1016/j.rse.2016.10.005 


\section{CHAPTER THREE}

\section{YIELD ESTIMATION OF SOYBEAN BREEDING LINES UNDER DROUGHT STRESS USING UNMANNED AERIAL VEHICLE-BASED IMAGERY AND CONVOLUTIONAL NEURAL NETWORK}

\subsection{Abstract}

Developing new crop varieties with improved traits through breeding programs is one of the key approaches to secure food supply for the growing global population of 10 billion in 2050. In breeding programs, yield is always used as a primary trait to select superior genotypes and evaluate breeding efficiency. However, yield trials have to be conducted at multiple locations for several years to evaluate the performance of numerous breeding lines and their adaptability to environments. The efficiency of conventional breeding programs is limited by the labor-intensive and time-consuming field phenotyping procedures, which can be improved by developing high-throughput field phenotyping systems using emerging technologies, including Unmanned Aerial Vehicle (UAV) imagery and deep learning technologies. The goal of this study was to evaluate the performance of a UAV imaging system and convolutional neural network (CNN) in estimating yield of soybean breeding lines. In this study, 972 soybean breeding plots in three maturity groups were planted under rainfed conditions for testing their drought tolerance. Aerial images were taken at the late vegetation (V6), early (R1), and late reproductive (R6-R8) growth stages. Seven image features associated with plant height, canopy color, and canopy texture were selected to estimate the yield of each breeding plot. A mixed CNN model was built to estimate soybean yield by taking the seven image features and two categorical factors, i.e. maturity group and drought tolerance, as predictors. Results show that image features collected at the early and late reproductive growth stages are comparably promising in 
estimating soybean yield. The prediction model could explain $78 \%$ of measured yield with a root mean square error of $391.0 \mathrm{~kg} \cdot \mathrm{ha}^{-1}$ (33.8 \% to average yield), indicating that the UAV imagery and deep learning models are promising in estimating yield for soybean breeding purposes.

Keywords: Soybean breeding; drought stress; yield estimation; UAV multispectral imagery; convolutional neural network

\subsection{Introduction}

Development of new crop varieties with improved traits through crop breeding programs is one of the promising solutions for the estimated food crisis in 2050 when agricultural production needs to double its current growth rate to feed 10 billion people in the world (Hincks, 2018). Soybean (Glycine max) seed has high protein and edible oil content and is an important and widely used crop for human food, animal feed, biofuel production, and many other products (Valliyodan et al., 2016). Global soybean production reached 336.11 million metric tons in 2019 (USDA-FAS, 2020) and world trade in soybean is projected to increase by 22\% in 2025 (Westcott \& Hansen, 2014). Desired crop traits for soybean include improved yield potential and other important agronomic traits such as disease resistance and stress tolerance, which can be obtained through breeding programs. Conventionally, a large number of progenies are made from a given population derived from a designed cross and grown in single-row plots. A certain percent of progenies (typically 10\%) are then selected based on visual evaluations of desired traits and grown at multiple locations over several years to further select a smaller group of genotypes that perform well under different environmental conditions (BATS Center, 1995; Sleper \& Poehlman, 2006). The conventional breeding procedure is labor-intensive and low efficient 
and may take seven to eight years to develop a small number of new soybean varieties from tens of thousands of progeny plots (Krueger, 2019).

Genetic gain is used to evaluate the efficiency of a breeding process (Fehr, 1991) and can be increased by increasing breeding population size, improving selection accuracy, and shortening the breeding cycle (Moreira et al., 2019). A larger population, including more progeny lines, more seeds, and larger fields, increases the chance of identifying the best combination of favorable alleles with high yield potential. However, the maximum population for a breeding program is limited by available labor, materials, resources, facilities, and time to handle all breeding lines. With the aid of accurate and automated high-throughput field phenotyping systems, breeders would be able to efficiently collect crop traits over a large population, evaluate soybean performance, increase selection intensity, and improve breeding efficiency (Araus et al., 2018). Leveraging emerging technology to predict yield in progenies or early breeding cycles will revolutionize the breeding program and improve the breeding efficiency significantly.

Recent studies in UAV-based plant phenotyping showed that phenotypic variances in crops can be quantified by one or combinations of image features, such as plant height (Feng et al., 2019), canopy cover (Chu et al., 2016), chlorophyll content (Kanning et al., 2018), stress symptoms (Zhou et al., 2020) and maturity dates (Zhou et al., 2019). Those image features representing important crop traits were found significantly correlating with crop yield. However, yield is a very complicated crop trait, and there are few studies conducted to predict the yield of soybean breeding lines in early breeding cycles according to a literature survey on Mar 3, 2020. As shown in Figure 3-1, there were 102 articles written in English related to the keywords of 'UAV' and 'yield prediction' or 'yield 
estimation' in the Scopus database (www.scopus.com), excluding reviews and erratum. Among them, there were 59 articles published during 2014-2020 that are related to crop yield, with 45 research papers were published in peer-reviewed journals and 14 were presented in conferences and symposiums. Most of the 59 articles attempted to prove the concept that UAV-based image features related to crop yield with $R^{2}$ (correlation of determination) from 0.4 to 0.92 . Some other studies were testing the potential use of image features in qualifying yield variations in single varieties of certain crops due to controlled experimental variables, such as irrigation, fertilization, or other management practices in field conditions. There is even less number of studies involving two or more crop varieties is even less (orange bars in Figure 3-1) among which only two studies directly estimated the yield of hundreds and more breeding materials using UAV imaging systems (Moreira et al., 2019; Yu et al., 2016).

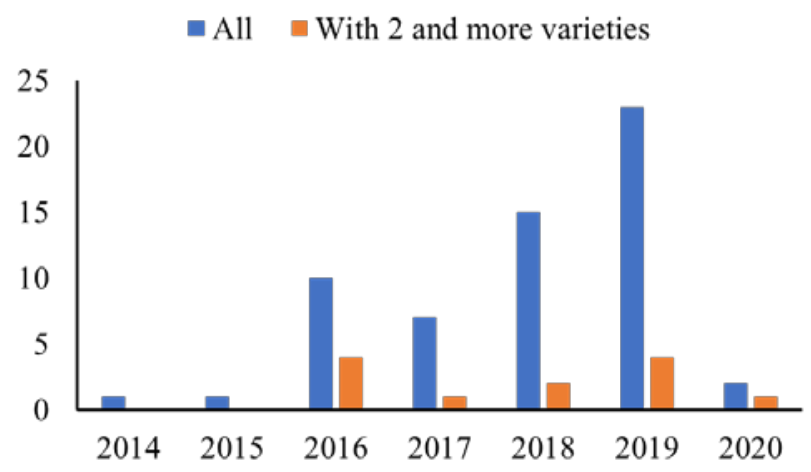

Figure 3-1. Publications about crop yield using UAV imagery.

The most widely used method for yield estimation is linear regression that has high interpretability and shows good performance in some cases. For example, Geipel et al. (2014) used a linear model to predict corn yield of one hybrid at four treatments of nitrogen fertilization using plant height and canopy coverage from UAV red-green-blue (RGB) images. The results showed that the prediction accuracy of yield $\left(R^{2}\right)$ reached up to 0.74 . 
However, prediction performance varied when linear models were applied to different scenarios even with similar predictors. For example, the yield prediction accuracy reported by Chu et al. (2016) was only up to 0.53 for predicting the yield of 35 cotton varieties using plant height and canopy coverage as predictors in their linear model.

Advanced data analytic methods, such as machine learning and deep learning, show great potential in processing complicated data sets and extracting hidden information of data that are not easily acquired using restrictive models (Elavarasan, et al., 2018). One of the examples is Partial Least Square Regression (PLSR) that was successfully used to estimate the yield of 20 commercial maize hybrids $\left(R^{2}=0.73\right)($ Herrmann et al., 2019) and 1103 soybean breeding lines $\left(R^{2}=0.59\right)$ (Zhang et al., 2019). Recently, deep learning methods have gained more and more attention thanks to high-performance computation systems. With the availability of convenient application programming interfaces (APIs), e.g. TensorFlow and Keras, more and more popular deep learning models, such as deep neural networks (DNNs), convolutional neural networks (CNNs), and recurrent neural networks (RNNs), have been used to address agricultural problems in four categories, i.e. identification of plant stresses, classification based on stress symptoms and signatures, quantification, and prediction of plant traits (Singh et al., 2018).

In breeding programs, tens of thousands of crosses from two parents are planted in progeny trials without replicates. Large variations in phenotypes (e.g. yield) can be caused by the conjunctions of their genetic and environmental variances (Fehr, 1991), consequently bringing large variances in image features and model predictions. Although a small percentage of selected genotypes are planted in following multi-location yield trials, the expansion of field networks across geographic locations increases spatial 
variances and consequently phenotypic variances in the same genotypes (Araus et al., 2018).

Yield estimation for breeding purposes is challenged by the complexity in genotypes and environments, and only a few studies have been reported. Maimaitijiang et al. (2020) predicted the yield of three soybean cultivars under two irrigation treatments with an $R^{2}$ of 0.72 and root mean square error (RMSE) of $1054.9 \mathrm{~kg} \cdot \mathrm{ha}^{-1}$ using a DNN based on RGB, multispectral, and thermal images. Ashapure et al. (2019) reported a result of $R^{2}=0.7-0.89$ for predicting the yield of a set of tomato genotypes using a simple Artificial Neural Network (ANN) model based on UAV-based RGB images. However, yield estimations for trials containing a large number of breeding varieties with unknown traits have not been reported.

This study was motivated by the need for accurate yield estimation of a large population of genotypes in soybean breeding programs for efficient selection of breeding lines with high yield potential. The goal of this study was to investigate the potential in estimating the yield of a large number of soybean breeding lines under drought stress using UAV multispectral imagery and a CNN model. The specific objectives were to (1) quantify the variance in yield due to environmental and genotypic variations in a typical breeding field trial setup, (2) evaluate the dynamics between soybean yield and image features collected at different growth stages, and (3) develop and evaluate a mixed CNN model for yield estimation.

3.3 Methods and materials

3.3.1 Field experiment and plant materials

A field experiment was conducted in a rainfed experimental field at Clarkton, 
Dunklin County, Missouri, United States in 2018. The experimental field locates in a humid subtropical climate region (Rubel et al., 2017) with sandy soil that facilitates moisture stress development in a few days after any rainfall event. The field has been used as an ideal location for drought research by the University of Missouri scientists for the last $10+$ years. Figure 3-2 b shows the weather conditions during the growing season, including daily average temperature and accumulative precipitation. The data were retrieved from a nearby weather station that is part of Missouri Mesonet - Weather Station Network (http://agebb.missouri.edu/weather).

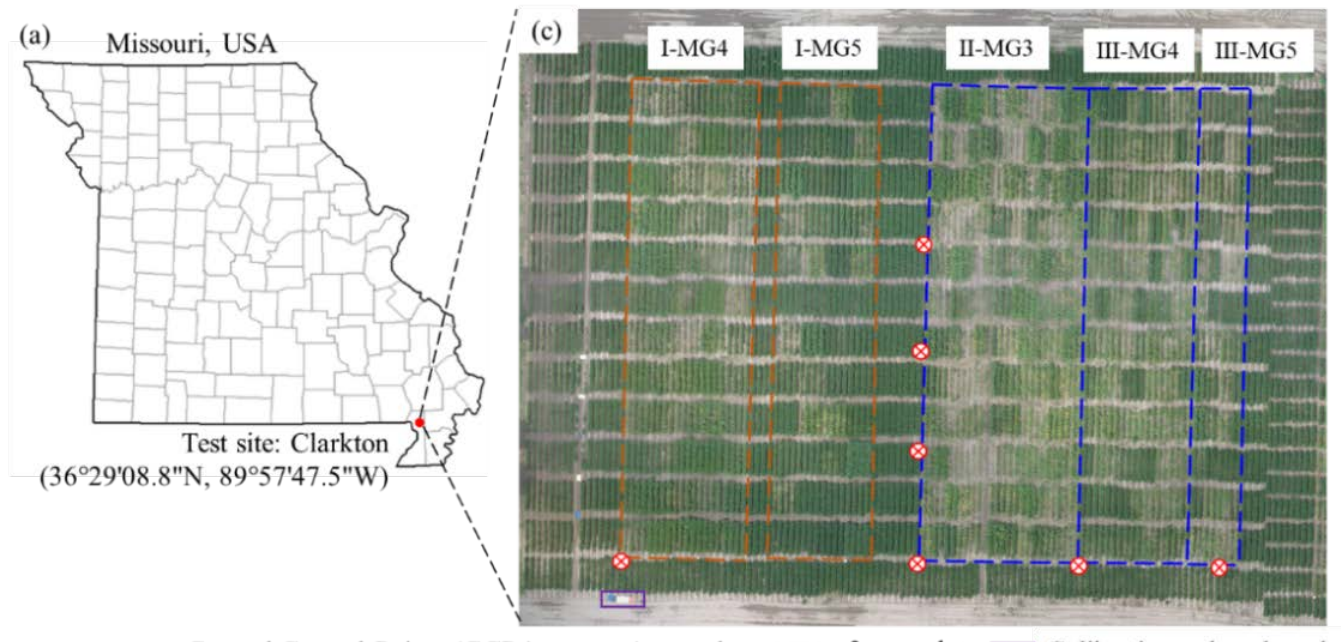

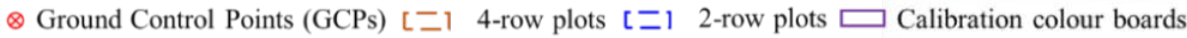

(b) $\square$ Daily Average Temperature -ө-Daily Cumulative Precipitation June $11 \quad$ July 72

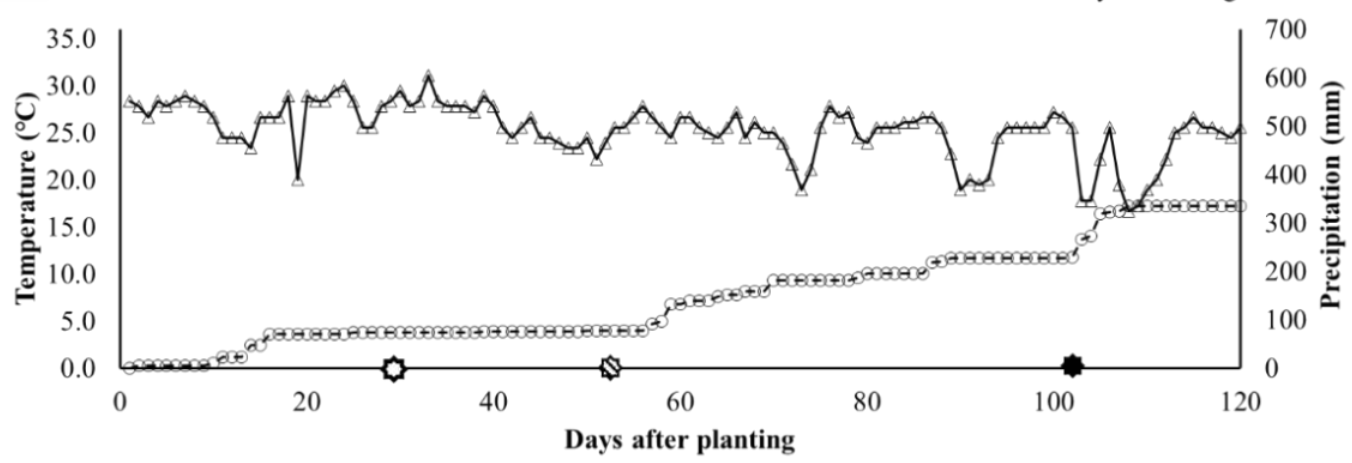

Figure 3-2. The field experiment. (a) The test site location. (b) Daily average temperature and cumulative precipitation during the growing season (c) Illustration of the experimental blocks in the field

The breeding field in this study was designed to develop drought tolerant soybean 
cultivars that are suitable for the mid-west and mid-south states of the USA. A group of 116 soybean advanced breeding genotypes and checks (commercial varieties) in maturity group (MG) 3, 4 and 5 were planted in three breeding blocks (Block I, II, III) of the field as shown in Figure 3-2 c. Block I included two breeding groups, i.e., I-MG4 and I-MG5. The I-MG4 group consisted of 15 advanced breeding lines, three commercial checks, one tolerant and one moderately susceptible check in MG 4. The I-MG5 group consisted of 11 advanced breeding lines, three commercial checks, one tolerant check and one susceptible check in MG 5. The Block II (II-MG3) contained 28 recombinant inbred lines (RILs) selected from two mappings derived from crosses involving one elite cultivar (drought susceptible) and one drought tolerant exotic soybean line. Four parental lines (drought susceptible and tolerant) involved in the crosses and four Near Isogenic Lines (NILs) bearing drought tolerance quantitative trait locus (QTLs) in MG 3. In addition, Block III also had two groups, with III-MG4 consisting of 29 varieties/lines in MG 4 and III-MG5 consisting of 12 varieties in MG 5 (proprietary cultivars of seed companies). Each group had one drought susceptible, one drought tolerant and one commercial check (P44A49X).

The soybean test entries in the Block I were planted in four-row plots, while those in the Block II and III were in two-row plots leading to 972 single-row soybean plots in total. All rows were $3.65 \mathrm{~m}$ in length and $0.75 \mathrm{~m}$ between rows. All breeding plots were planted on May 8, 2018, with three replications following a randomized complete block design. The test field was irrigated once at the vegetative stage (V6) to prevent a foreseeable death of soybean plots due to the severe drought. Plots were harvested using a plot combine during mid-October 2018. The seed yield of each plot was calculated based on the weight of two center rows of the four-row plots or all two rows of the two-row plots 
in kilograms per hectare $\left(\mathrm{kg} \cdot \mathrm{ha}^{-1}\right)$. Under the drought environment, the average yield of all the experimental materials was $1156.8 \mathrm{~kg} \cdot \mathrm{ha}^{-1}$.

\subsubsection{Visual wilting scores of soybean plots}

Canopy leaf wilting is one of the most important visual symptoms that are used by breeders to determine the drought-tolerant soybean varieties (Carter Jr, 1999; Pathan et al., 2014). The drought-sensitive plants wilt quickly (referred as fast-wilting) while the tolerant plants show delayed (referred as slow-wilting) canopy wilting under drought stress. Genotypes with the slow-wilting trait were found yielding more than those with the fastwilting trait under drought stress environments (Valliyodan et al., 2016).

The canopy wilting was rated visually during 1-3 pm in the first week of July 2018 when the field was experiencing drought stress and plants showed noticeable canopy wilting symptoms. Canopy wilting was rated on a scale of 1 to 5 using the method described in Zhou et al. (2020). The slow and fast-wilting genotypes were classified by taking 2.5 as a midpoint, i.e., genotypes with an average wilting score (mean of three replicates) lower than 2.5 were categorized in the slow-wilting group and those with scores higher than or equal to 2.5 were in the fast-wilting group (Ye et al., 2019). Slow- and fast-wilting genotypes of three MGs were scored resulting in six groups with two factors $(\mathrm{MG} \times$ wilting traits). The number of soybean breeding plots in each group is shown in Table 3-1.

Table 3-1. The number of soybean breeding plots in the six groups (3 MGs $\times 2$ wilting traits).

\begin{tabular}{lcccc}
\hline \multirow{2}{*}{ Wilting traits } & \multicolumn{3}{c}{ MG } & \multirow{2}{*}{ Total } \\
\cline { 2 - 4 } & 3 & 4 & 5 & \\
\hline Slow & 110 & 176 & 152 & 438 \\
Fast & 112 & 256 & 166 & 534 \\
Total & 222 & 432 & 318 & 972 \\
\hline
\end{tabular}




\subsubsection{UAV imagery data collection}

Imagery data were acquired using a UAV imaging system consisting of a UAV platform (model: DJI Matrice 600 Pro, DJI, Shenzhen, Guangdong, China) and two types of cameras, i.e., a multispectral and a visible RGB camera. Data were collected on June 11, July 7 and Aug. 22, 2018 when soybeans were at the late vegetation stage (V6), the early flowering stage (R1) and late reproductive stages (R6-R8). The RGB images were taken using a digital camera (GoPro Hero 5 Black, GoPro. Inc., San Mateo, CA, USA). It has a resolution of $4000 \times 3000$ pixels and was set to take time-lapse photos at 2 fps (frame per second). The multispectral images on June 11 and July 7 were acquired using a customized camera that was modified by replacing the spectral filter in the blue channel of a digital camera (PowerShot ELPH 300, Canon U.S.A., Inc., Huntington, NY, USA) with a nearinfrared (NIR) filter (800-900 nm) (MaxMax.com - Llewellyn Data Processing, Carlstadt, NJ, USA). The multispectral camera has a resolution of $4608 \times 3456$ pixels and was controlled with a third-party firmware Canon Hack Development Kit (CHDK, http://chdk.wikia.com) to take images at 0.5 fps. The multispectral images on Aug. 22 were acquired using a five-channel (Blue-Green-Red-RedEdge-NIR) camera (model: RedEdgeM, MicaSense, Seattle, WA, USA) that has a resolution of $1260 \times 960$ pixels. The fivechannel camera was configured to take time-lapse images at $1 \mathrm{fps}$ and calibrated using a spectral standard board before flights following the procedure described in Zhou et al. (2019).

The UAV imaging system flew at $15 \mathrm{~m}$ above ground level at a nadir view for all data collections, resulting in ground sampling distances (GSDs) of 7.7, 4.7 and 10.4 $\mathrm{mm} \cdot \mathrm{pixel}^{-1}$ for the RGB, three-channel multispectral and five-channel multispectral 
images, respectively. Prior to the flights, the flight speed was set to $7 \mathrm{~km} \cdot \mathrm{h}^{-1}$ and the flight paths were designed to ensure a forward overlap of $\geq 70 \%$ and side overlap of $\geq 65 \%$ for all images using a flight control App Autopilot (Hangar Technology, Austin, TX, USA).

Seven ground control points (GCPs) were placed in the field in each data collection. The GCP was made of a $30 \times 30 \mathrm{~cm}$ wood square covered with a cross-patterned vinyl sheet that was mounted on the top of a $1.1 \mathrm{~m}$ plastic fence post. The GCPs were manually stepped into the soil before each data collection and each had a height of $99 \mathrm{~cm}$ above the ground. In addition, the plant height of nine randomly selected soybean plots was manually measured from the ground to the base of the highest fully expanded leaf in every data collection. The measured plots were identified in images using the location information on their labels.

\subsubsection{Image processing}

Orthomosaic images and digital elevation models (DEMs) of the field were generated using the software Agisoft PhotoScan Pro (Agisoft LLC, St. Petersburg, Russia), following the procedures described in Zhou et al. (2019). The DEM was built based on the principle of structure from motion (SfM) and included elevation information (relative to mean sea level) of soybean canopy, bare soil surface and GCPs (Feng et al., 2019). The geo-reference information of the five-channel multispectral images was obtained using the GPS information from their metadata. The geo-reference information of the RGB and three-channel multispectral images was manually assigned using the telemetry data of UAV flight logs. The orthomosaic images and DEMs were then processed using the Image Processing Toolbox and Computer Vision System Toolbox of MATLAB (ver. 2019a, The MathWorks, Natick, MA, USA). 
The pixel values of orthomosaic images from RGB and multispectral images are linearly correlated to the light intensities that are closely related to imaging time (Yu et al., 2016). To make the data collected at different dates comparable, all images were calibrated using the calibration color boards (Figure 3-2 c) included in each data collection. Using images collected on June 11 as a baseline, linear equations for each image channel were obtained by regressing digital numbers of the black and blue color calibration boards. The linear equations were applied to the images collected on other dates.

Individual soybean plots were separated from the orthomosaic and DEMs by manually drawing vertical and horizontal lines in alleys between plots and then resized to $20 \times 100$ (the input size of a mixed CNN model). Soybean plants were segmented from the background by detecting projected canopy contours using the 'activecontour' function (Whitaker, 1998) with the 'Chan-Vese' method (Chan \& Vese, 2001) and 400 maximum iterations. Pixels within a full contour were considered as foreground (soybean plants) while those outside contours were background (soil and residues). Contours with extremely small regions were identified as noises using the 'regionprops' function and then removed from the foreground.

Six image features, including Hue $(\mathrm{H})$ and Saturation (S), plant height and three VIs, were extracted to evaluate the best growth stages in estimating yield. The image features $\mathrm{H}$ and $\mathrm{S}$ were extracted from the HSV color space converted from the RGB orthomosaic images using the function 'rgb2hsv' of MATLAB. The HSV color space has attributes corresponding directly to the basic color concepts, which makes it conceptually simple in image processing (Shipman, 2012). The height of soybean plots at three dates was derived from the DEMs built from RGB images. Outliers in each DEM of soybean 
plots and the GCPs were detected and removed using the 'rmoutliers' function with the default options. Height of the GCPs and plant was then calculated as the difference between the $95 \%$ percentile of the DEM (considered as the elevation of plants or GCPs) and the 5\% percentile of the DEM (considered as the elevation of ground/soil) of each plot. Three VIs, including Normalised Difference Vegetation Index (NDVI), green NDVI (gNDVI) and Excess Green (ExG), were calculated from the processed three-channel and five-channel multispectral images using Eq. 3-1 to 3-3.

$$
\begin{gathered}
N D V I=\frac{N I R-R}{N I R+R} \\
g N D V I=\frac{N I R-G}{N I R+G} \\
E x G=2 \times G-R-B
\end{gathered}
$$

where, NIR, R and G are the digit number of each image pixel acquired by the multispectral cameras. The VIs were designed to combine two or more spectral bands to signify vegetation and suppress non-vegetation objects in the images.

\subsubsection{Data analysis}

All data analyses were performed in RStudio (Ver. 1.1.414, RStudio, Boston, MA, USA). A two-way analysis of variance (ANOVA) with an honest significant difference (HSD) Tukey test was conducted to evaluate the effects of MG, wilting trait, and their interactions on yield. A one-way ANOVA was conducted to compare the changes in image features over the three growth stages. ANOVA tests were conducted at a significance level of 5\% using the ' $a o v$ ' function and the Tukey tests were performed using the 'TukeyHSD' function. The correlation between each image feature and yield was calculated using the 'corr' function in RStudio. 


\subsubsection{Yield estimation using a mixed CNN model}

A mixed CNN model was designed to estimate the yield of each soybean breeding plot. Predictors of the model included seven input images extracted from the processed images for each soybean plot as well as two vectors containing the categorical attributes of MG and wilting traits associated with the soybean plots. Manually measured yield data were used as training outputs to the model.

\section{(1) Architecture of the mixed CNN model}

The architecture of the mixed CNN model is illustrated in Figure 3-3, which consists of two branches with one (Branch 1) taking the seven input images and another (Branch 2) taking the categorical vectors as inputs. Each of the branches generated a vector of outputs. The two vectors were concatenated into one single vector that was directly connected to the output layer. Branch 1 (Figure 3-3) consisted of 20 layers to process the seven input images for each soybean plot. The input images were processed using six convolutional layers with a number of randomly initialized filters each with a size of [3, 3] in each layer. The numbers of filters were incremented exponentially to generate more sophisticated filters as the network goes deeper. A Rectified Linear Unit (ReLU) activation function that defines the output of neurons given a set of inputs from previous layers was selected for each convolutional layer. All the convolutional outputs less than 0 will be transformed to 0 , while those greater than 0 will be outputted themselves (LeCun et al., 2015). A batch normalization layer was then introduced to prevent arbitrary parameters (weights and biases) that were added in the convolutional layers from being imbalanced with extremely high or low values. It was proved that batch normalization can greatly increase the speed and training accuracy and reduce the influence of the outliers with large 
weight (Ioffe \& Szegedy, 2015). An average pooling layer was added after every two batch normalization layers to reduce the dimensionality of the output from previous layers.

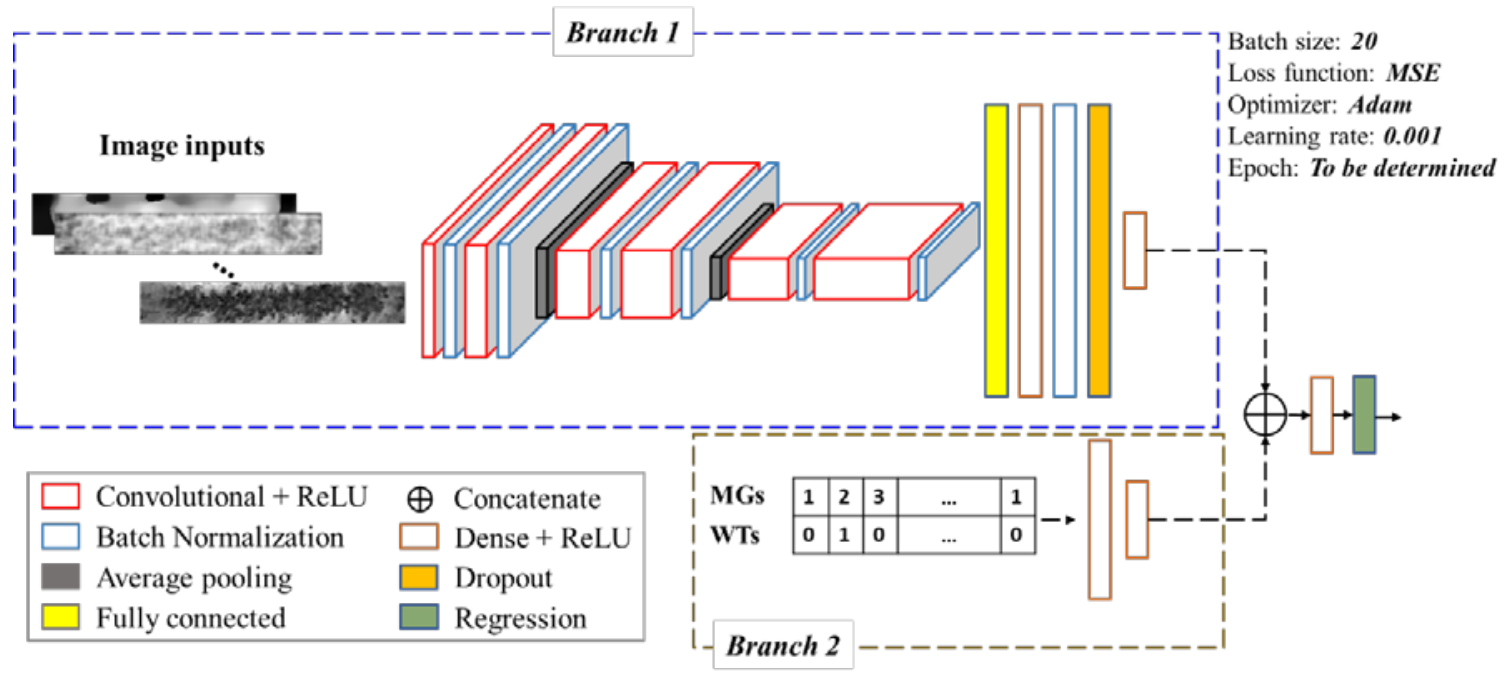

Figure 3-3. The architecture of the mixed CNN model.

A fully connected layer was then added to the end of all the convolutional layers to transform 2D image features of each soybean plot into a 1D vector that can be connected to their yield. A dense layer with the ReLU activation was used to further create trainable parameters related to the vector. To prevent overfitting, a dropout layer was added to randomly ignore the neuron units that could be possibly co-dependent on each other. Finally, another dense layer was added to match the number of nodes from both branches.

In Branch 2 (Figure 3-3), two categorical vectors each with the size of $1 \times n$ ( $n$ is the number of soybean plots) contained codes for MG and wilting traits of each soybean sample. A dense layer with 16 kernels (weight matrices) and the ReLU function were used to activate the categorical vectors with a set of trainable parameters, followed by a dense layer with four kernels. After trained by two dense layers, the categorical outputs were concatenated with the image outputs and connected to the regression layer.

The 972 soybean plots were randomly split into training (70\%) and testing (30\%) 
datasets to train and validate the model during the training process. The parameters associated with all layers were trained and optimized iteratively according to the setup of the model's hyperparameters (the top right corner of Figure 3-3). In flexible modelling methods such as CNN models, overfitting commonly happens during model training when model parameters are tuned to closely fit the training set and the training loss is much lower than the testing loss (James et al., 2013). Overfitting could be prevented by carefully monitoring the training epochs (Goodfellow et al., 2016). The optimal number of epochs was determined when the testing loss stops decreasing while the training loss keeps increasing. The mixed CNN model was developed in Keras (https://keras.io/), an opensource deep learning API written in Python.

(2) Data set preparation

Images features extracted from the 5-band multispectral images on Aug 22 (R6-8) were used as predictors to the mixed CNN model. The input for each soybean plot has seven images, including the red edge (705-729 nm) band of the multispectral images, three VIs (Table 3-2), a DEM and two texture features that were used to quantify the roughness or smoothness of soybean canopy, including contrast and entropy. The image texture features were calculated based on CVI (Table 3-2) using the 'graycomatrix' function in MATLAB.

Table 3-2. Descriptions of three vegetation indices and texture features used in the mixed CNN model. The variables in the formulas of VIs are the pixel values of different channels of the 5band multispectral. $\mathrm{p}_{\mathrm{ij}}$ is the pixel value of the $\mathrm{i}^{\text {th }}$ row and the $\mathrm{j}^{\text {th }}$ column in an image. $\mathrm{N}$ is the dimension of the moving window (kernel) when calculating the grey level co-occurrence matrix of each image for texture features

\begin{tabular}{ccc}
\hline Index Name & Full name & Formula \\
\hline CCCI & Canopy Chlorophyll & Content Index \\
(Barnes et al., 2000) & $\frac{\mathrm{NIR}-\mathrm{Re}}{\mathrm{NIR}+\mathrm{Re}}$ & $\mathrm{NDVI}$
\end{tabular}


CVI

(Hunt et al., 2011)

MTVI2

(Haboudane et al., 2004)

Contrast

(Hall-Beyer, 2017)

Entropy

(Hall-Beyer, 2017)
Chlorophyll VI

Modified Triangular VI

$$
\begin{gathered}
\sum_{i, j=1}^{N} p_{i, j}(i-j)^{2} \\
\sum_{i, j=1}^{N} p_{i, j}\left(-\ln p_{i, j}\right)^{2}
\end{gathered}
$$$$
1.5 \times(1.2 \times(\mathrm{NIR}-\mathrm{G})-2.5 \times(\mathrm{R}-\mathrm{G}))
$$$$
\sqrt{(3-\mathrm{NIR})^{2}-6 \times \mathrm{NIR}+5 \times \sqrt{\mathrm{R}}-0.5}
$$

The model datasets, i.e. images as input and yield as output, were normalized to balance the training parameters and improve converge efficiency (Moghimi et al., 2020). To avoid removing relative differences between images of different soybean plots, the image normalization was conducted for each channel separately using the mean and standard deviation of image pixel values of all soybean plots in that channel, as shown in Eq. 3-4 (Freedman et al., 2007).

$$
p^{\prime}{ }_{i j n k}=\frac{p_{i j n k}-\mu_{k}}{\sigma_{k}}
$$

where $p_{i j n k}$ is the pixel value of the $i^{\text {th }}$ row and the $j^{\text {th }}$ column in an image of the $n^{\text {th }}$ soybean plot in the $k^{\text {th }}$ channel, $p^{\prime}{ }_{i j n k}$ is the normalized value of $p_{i j n k}, \mu_{k}$ is the mean and $\sigma_{k}$ is the standard deviation of the image pixel values of all soybean plots in the $k^{\text {th }}$ channel. Yield data were normalized using Eq. 3-5 to mitigate the slight right skewness in its distribution (Kutner et al., 2005).

$$
y_{n}^{\prime}=\frac{\sqrt{y_{n}}-\mu}{\sigma}
$$

where $y_{n}$ is the original yield for the $n^{\text {th }}$ soybean plot, $y_{n}^{\prime}$ is the normalized yield for the $n^{\text {th }}$ soybean plot, $\mu$ is the mean and $\sigma$ is the standard deviation of the square root yield of all soybean plots. $\mu=32.46$ and $\sigma=10.82$ in this case. 


\subsection{Results and discussion}

\subsubsection{Accuracy of plant height from DEM}

The measurement accuracy of DEMs was evaluated by comparing the DEM-based height measurement with the rated height of the GCPs and manually measured height of soybean plants. As shown in Figure 3-4a, the overall measurement RMSE of the height for the GCPs was $2.9 \mathrm{~cm}$ in all three data collections. The largest error was $8 \mathrm{~cm}$ for the sixth GCP collected on June 11, which might because the fence post was not fully stepped into the dry soil so that its actual height was larger than $99 \mathrm{~cm}$. In addition, uneven ground surface or tilt GCPs may have caused errors in the height from DEMs.

(a)

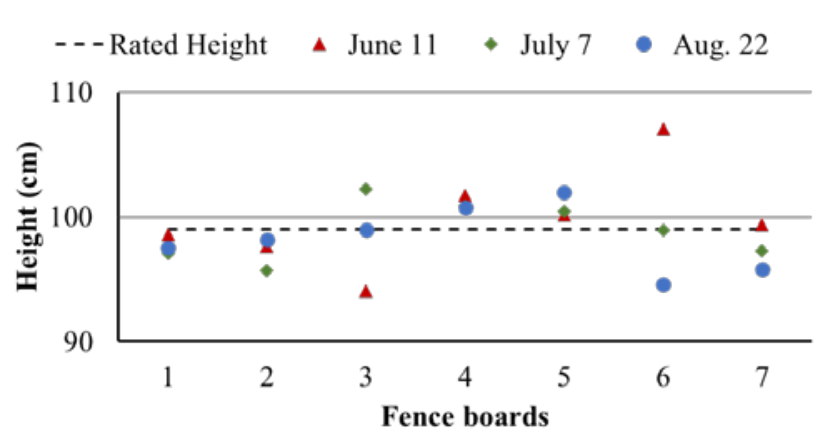

(b)

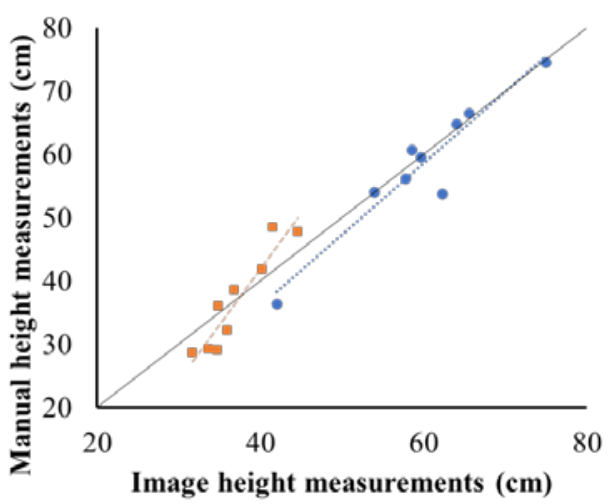

Figure 3-4. Comparison of the height from DEMs with manual measurements. (a) The deviations of the GCPs height from DEM. (b) Agreement of the height from DEMs and manual measurement of nine soybean plots collected on July 7 and Aug. 22. Black solid line: a reference line $(y=x)$. Orange dash line: a least square linear fit of the orange dots $(y=1.8 x-28.5)$. Blue dot line: a least square linear fit of the blue dots $(y=1.1 x-9.1)$.

Figure 3-4 b shows the agreement between the DEM-derived and manually measured plant height of nine soybean plots on July 7 and Aug. 22. In general, both data had high agreements with $R^{2}=0.88$ and 0.90 for the data on July 7 and Aug. 22 with the relative $R M S E$ to the average of $10.8 \%(\mathrm{RMSE}=4.0 \mathrm{~cm})$ and $6.1 \%(\mathrm{RMSE}=3.6 \mathrm{~cm})$, respectively. It was noticed that the measurement errors of soybean plots were larger than 
those of the GCPs, which might be due to the greater height variations in individual soybean plots than these of the GCPs with flat surfaces, and it might also be caused by personal biases during manual operations. Additionally, plant heights from the DEM on July 7 were overestimated in the lower range, but underestimated for the higher range, compared to the manual measurements of these nine soybean plots. The errors could result from the difficulties in both generating DEMs and keeping human slights as horizontal as soybean canopies on short objects $(28-48 \mathrm{~cm})$ in field conditions. The adequacy of using plant height from the DEM at the early stages will be further evaluated using lower standard objects (GCPs) during data collection.

\subsubsection{The effects of the maturity and wilting trait on yield}

The results of the two-way ANOVA test (Figure 3-5) show significant effects of maturity, wilting trait, and their interactions on soybean yield $(\mathrm{p}<0.001)$. Significant differences were also observed in yield between two wilting groups across the three MGs, with the slow-wilting group producing on average $1395.0 \mathrm{~kg} \cdot \mathrm{ha}^{-1}$ and the fast-wilting group only $986.5 \mathrm{~kg} \cdot \mathrm{ha}^{-1}$ (Figure 3-5a). Such yield benefit from the slow wilting trait has also been noted in other studies (Pathan et al., 2014; Ye et al., 2019) and is likely associated with other underlining physiological traits. Specifically, it has been observed that higher production resulted from the complex drought tolerance mechanism behind the symptom of slow canopy wilting, including the uptake of water from deep soil horizon, improving water use efficiency, and limiting transpiration rate (Passioura, 2007; Sadok \& Sinclair, 2010). 
(a)

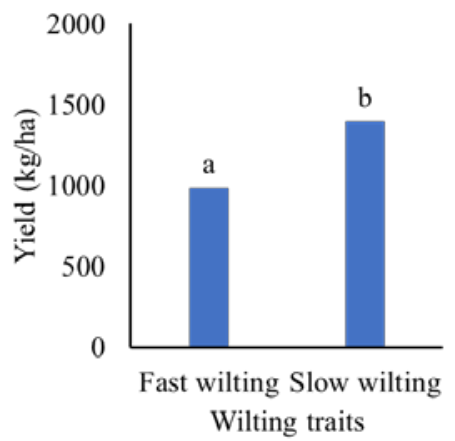

(b)

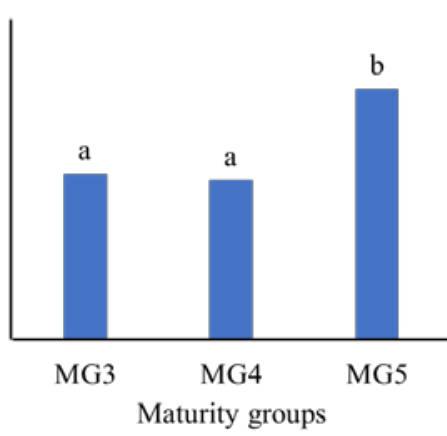

(c) $\square$ Fast wilting $\square$ Slow wilting

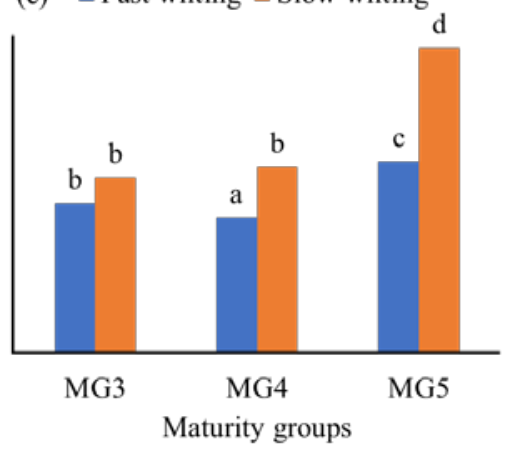

Figure 3-5. The bar plots of the effects of maturity group, wilting trait, and their interactions on yield. (a) is the yield means of the fast- and slow-wilting groups. (b) is the yield means of three MGs. (c) is the yield means of three MGs with different wilting traits. The lower-case letters above bars indicate the significant difference among these means at a 0.001 significance level.

No significant difference was found in yield between MG $3\left(1018.0 \mathrm{~kg} \cdot \mathrm{ha}^{-1}\right)$ and MG $4\left(976.8 \mathrm{~kg} \cdot \mathrm{ha}^{-1}\right)$ across wilting traits, while the yield of MG $5\left(1540.0 \mathrm{~kg} \cdot \mathrm{ha}^{-1}\right)$ was significantly higher than that of MG 3 and MG 4 (Figure 3-5 b). Generally, soybean genotypes in later MGs, thus with extended maturity time may take full advantage of the growing season to improve their yield (Zhou et al., 2019). The longer growing period of MG 5 plots may offer opportunities and time for plants to recover from stress conditions such as drought, thereby resulting in higher yields. The interactive effects of MGs and wilting traits were also significant on yield, implying that reductions in yield loss due to soybean drought tolerance depend on its maturity stages, i.e., the stage that soybeans are under water stress. The finding is similar to the conclusion drawn by Kron et al. (2008) that responses of soybean differed to water stress induced at different stages. In addition, Desclaux et al. (2000), and Rosolem et al. (2005) also observed that the growth period of pod initiation (R3) to 50\% yellow leaves (R7) is the most critical stage for productivity and thus soybean showed greater susceptibility to water stress during these stages. In our case, the absence of yield improvement of the MG 4 genotypes (Figure 3-5 b) might be due to the average yield loss caused by the severe drought during their reproductive stages. 


\subsubsection{Image features collected at three growth stages}

ANOVA tests were conducted to evaluate their variations on different growth stages based on the six image features extracted from two types of images. Image features included three RGB derived features (hue, Saturation, and ExG), two VIs related to the NIR band (NDVI and gNDVI), and a dimensional parameter (height). The results of ANOVA as shown in Figure 3-6 show a significant difference among each of these image features collected at three growth stages. It can be seen from Figure 3-6a that the mean $\mathrm{H}$ of all soybean plots collected on July 7 was significantly higher than both earlier and later dates. Hue resembles pure colors and the range from 0.15 to 0.40 in Figure 3-6a covers the colors from yellow-green to dark-green (Smith, 1978). Soybean plants were in light green color at vegetative stages (extreme low values could be due to remaining soil background around small plants) and reached the highest green (hue) during reproductive stages (full canopy coverage). The decrease in hue at later stages might be because the more seed pods achieved mature color, i.e. grey, tan or brown color as soybean entered maturity status (Zhou et al., 2019). 

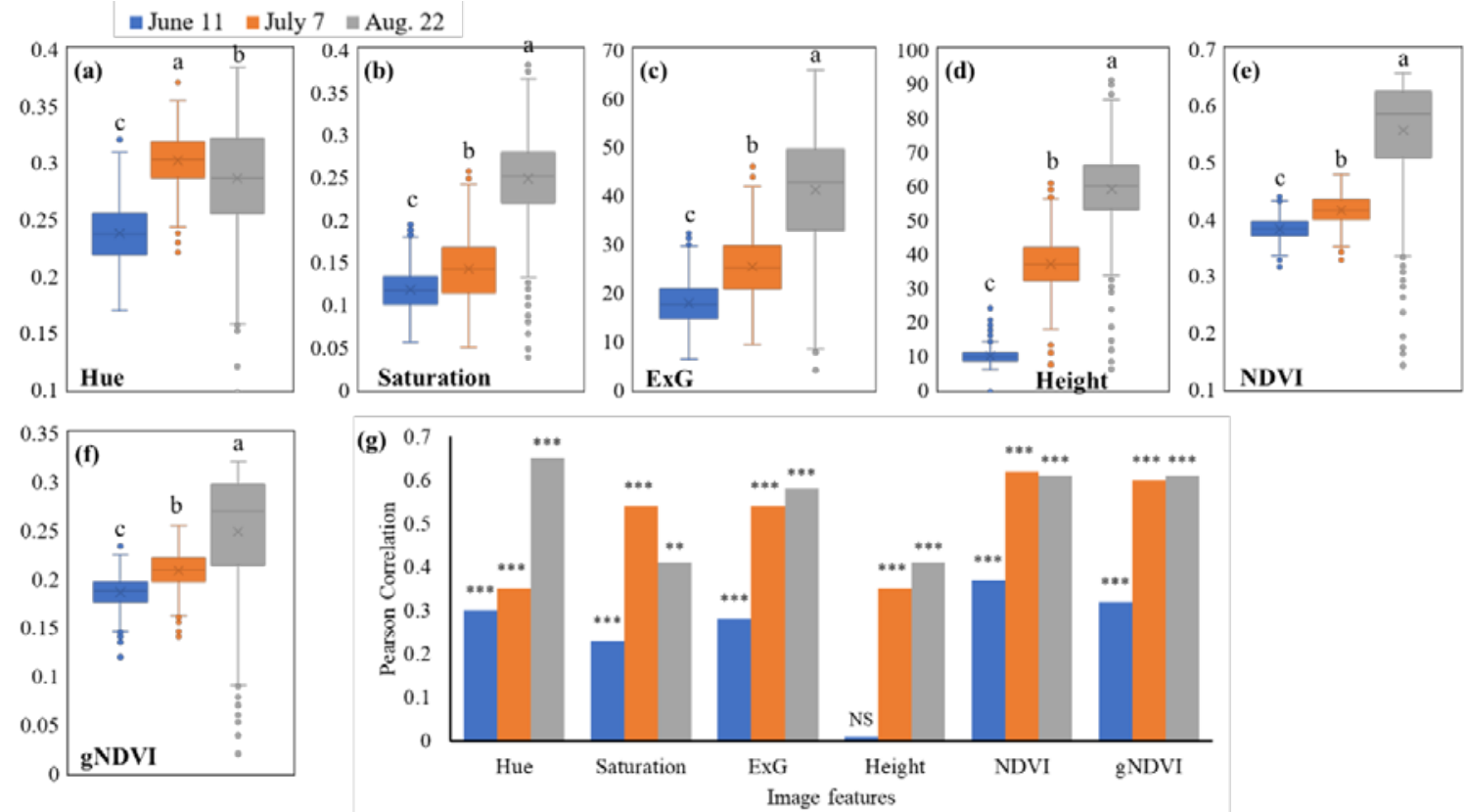

Figure 3-6. Image features collected at three growth stages. (a) - (f) are boxplots of six image features, i.e. hue, saturation, ExG, height, NDVI, and gNDVI collected at three growth stages.

The lower-case letters indicate the significant difference among these means at a 0.05 significance level. (g) Pearson correlations between yield and image features collected at three dates. NS indicates No Significance. ${ }^{* *},{ }^{* * *}$ indicate the significance levels of $p=0.01$ and 0.001 , respectively.

In addition, Figure 3-6 b and f show that the mean values of the other five image features gradually increase as soybean maturing. It is straightforward to understand that soybeans were averagely taller at later stages (no lodging was observed in this experiment). It should be noted that soybean height at the latest stage varied more than earlier stages Figure 3-6 d, which might be due to the genotype variations as well as water stress induced in the late vegetation stage (V4) (Desclaux et al., 2000). As NDVI and gNDVI are widely used to quantify chlorophyll content in plants (Gago et al., 2015; Gitelson \& Merzlyak, 1996), Figs. 3-6 e and f imply that soybeans at later stages had higher chlorophyll generally. It was observed by Zhou et al. (2019) that mature soybean plots had significantly lower NDVI values than immature plots, leading to high variations in NDVI and gNDVI values.

Pearson correlations between yield and each of the image features collected were 
calculated to evaluate the potential growth stages in estimating yield (Figure 3-6 g). Except for plant height on June 11, all other image features at three dates had significant positive relationships with yield. Image features on Aug. 22 had the best overall performance with $r$ ranging from $0.41-0.65$, followed by the features on July 7 with $r$ from $0.35-0.62$, and those collected on the earliest stage presented the poorest performance with $r$ from 0-0.37. On June 11, soybean plants were at the V6 stage and started experiencing drought after 18 days without any precipitation and irrigation. As yield could be severely reduced by drought conditions during the flowering stage by limiting the number of pods produced per plant and seed filling stages by reducing seed weight (Corn Agronomy, 2015; Desclaux et al., 2000), image features at the early stages might not be reliable to predict yield.

It should be noticed that S and NDVI on July 7 had higher $r$ values compared to those on Aug. 22, and two $r$ values for gNDVI on both dates were surprisingly close to each other. Additionally, right skewness that might underestimate $r$ in linear regressions (Kutner et al., 2005) can be observed from the box plots of the three image features on Aug. 22. It is possibly because the genotypes in early MGs had entered the maturity status (95\% of pods achieved mature pod color) and halted parts of their physiological changes (Yu et al., 2016), leading to the aggregation at the top of boxes. Therefore, the comparison suggests that image features collected at the early and late reproductive stages are comparably promising in estimating soybean yield.

Although strong linear correlations between image features and yield were observed in similar studies for breeding purposes (Maja et al., 2016; Yu et al., 2016), the performance of yield estimation was limited using specific image features with single data points representative for soybean plots. Underlying features beyond the commonly used 
features were expected to be explored using more complicated models, e.g. CNN models.

\subsubsection{Yield estimation using the mixed CNN model}

The mixed CNN model was trained for 100 epochs and the results are shown in Figure 3-7. The model loss for the training and testing set (Figure 3-7 a) generally decreased over epochs. It was noticed that the testing loss exhibited a slightly increasing trend at around the 60th epoch, while training loss kept decreasing, which may be caused by overfitting (Ashapure et al., 2019). Therefore, the $60^{\text {th }}$ epoch was considered as an optimal point for training the model. The performance for yield estimation with the optimal mixed CNN model achieved an $R^{2}$ of 0.78 with the RMSE of $391.0 \mathrm{~kg} \cdot \mathrm{ha}^{-1}$ (33.8 \% to average yield).

The CNN branch of the mixed model (Branch 1) was trained separately for 50 epochs to compare its performance with the mixed model, i.e. comparing the performance between the estimations with and without the categorical factors. As shown in Figure 3-7 c and d, the estimation performance of the CNN branch yielded a slightly lower $R^{2}(0.76)$ and higher RMSE (439 kg $\cdot \mathrm{ha}^{-1}$ ) than the mixed model, implying the necessity of including MGs and wilting traits as categorical factors in the model. 

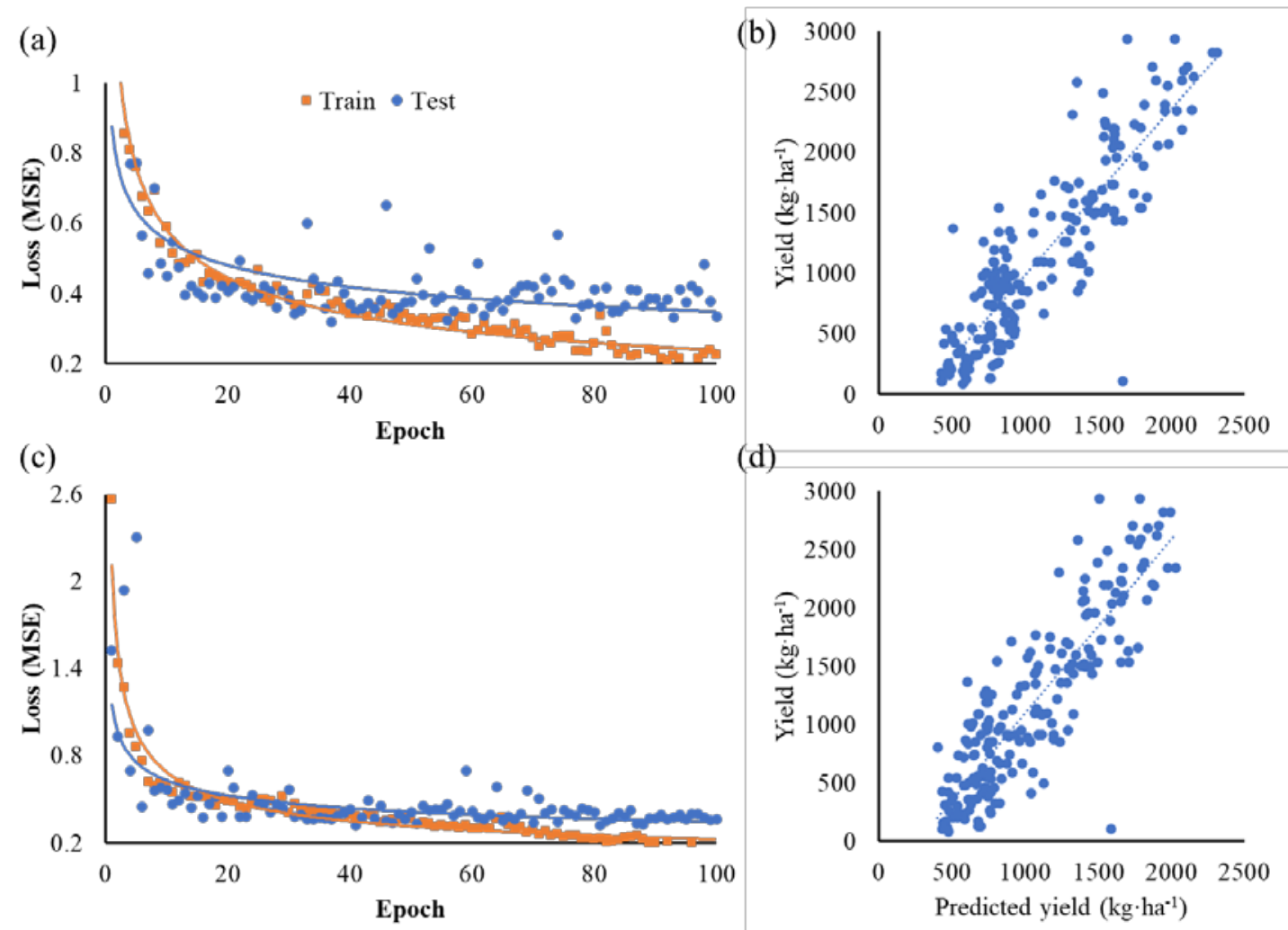

Figure 3-7. The performance of yield estimated by the CNN branch and the mixed CNN model with seven image features as predictors. (a) and (c) are the scatter plots of losses against epochs. (b) and (d) are the scatter plots of the predicted vs. the measured yield. Blue dot lines: least square linear fits of the dots $(y=1.4 x-393$ and $y=1.5 x$-399 for the lines in (b) and (d), respectively). (a) and (b) are for the mixed CNN model. (c) and (d) are for the CNN model.

The proposed mixed CNN model is competitive among similar studies that focused on yield estimation for soybean breeding purposes. For example, the estimation accuracy with $R^{2}=0.72$ and $R M S E=1054.9 \mathrm{~kg} \cdot \mathrm{ha}^{-1}$ was reported by Maimaitijiang et al. (2020) for predicting the yield of three soybean cultivars under two irrigation treatments. Zhang et al. (2019) reported an estimation accuracy of $R^{2}=0.59$ for 1103 soybean breeding lines using a PLSR model and image features from hyperspectral imagery. Based on personal communication with soybean breeders, the estimation accuracy is an important factor to improve the selection intensity of superior soybean lines. The evidence in this study that image features at early reproductive stages have great potential in estimating yield will help breeders to adjust experimental cycles in future trials and thus accumulatively improve the 
efficiency of soybean breeding programs. The framework and proof of concept developed in this study may provide evidence of yield estimation using key image features and a mixed CNN model, which might be potentially useful in differentiating yield components in crops for other studies.

Figure 3-8 lists images of five soybean plots that had the yield of 235.4, 853.4, 1537.4, 2346.9 and $2836.3 \mathrm{~kg} \cdot \mathrm{ha}^{-1}$ from the top to bottom. They were divided into five levels from L0 to L4 corresponding to their yield levels. From the RGB image in Figure 3$8 \mathrm{a}$, it is observed that soybean plots with higher yield had a darker green color canopy. As chlorophyll content has shown a positive relationship with yield in rice (Liu et al., 2017) and wheat (Kanning et al., 2018), the darker green might imply higher chlorophyll content in a soybean plot. In addition, MTVI2, CCCI and CVI are VIs related to chlorophyll content (Barnes et al., 2000; Vincini et al., 2008), the darker green canopy had higher values in these channels too. 


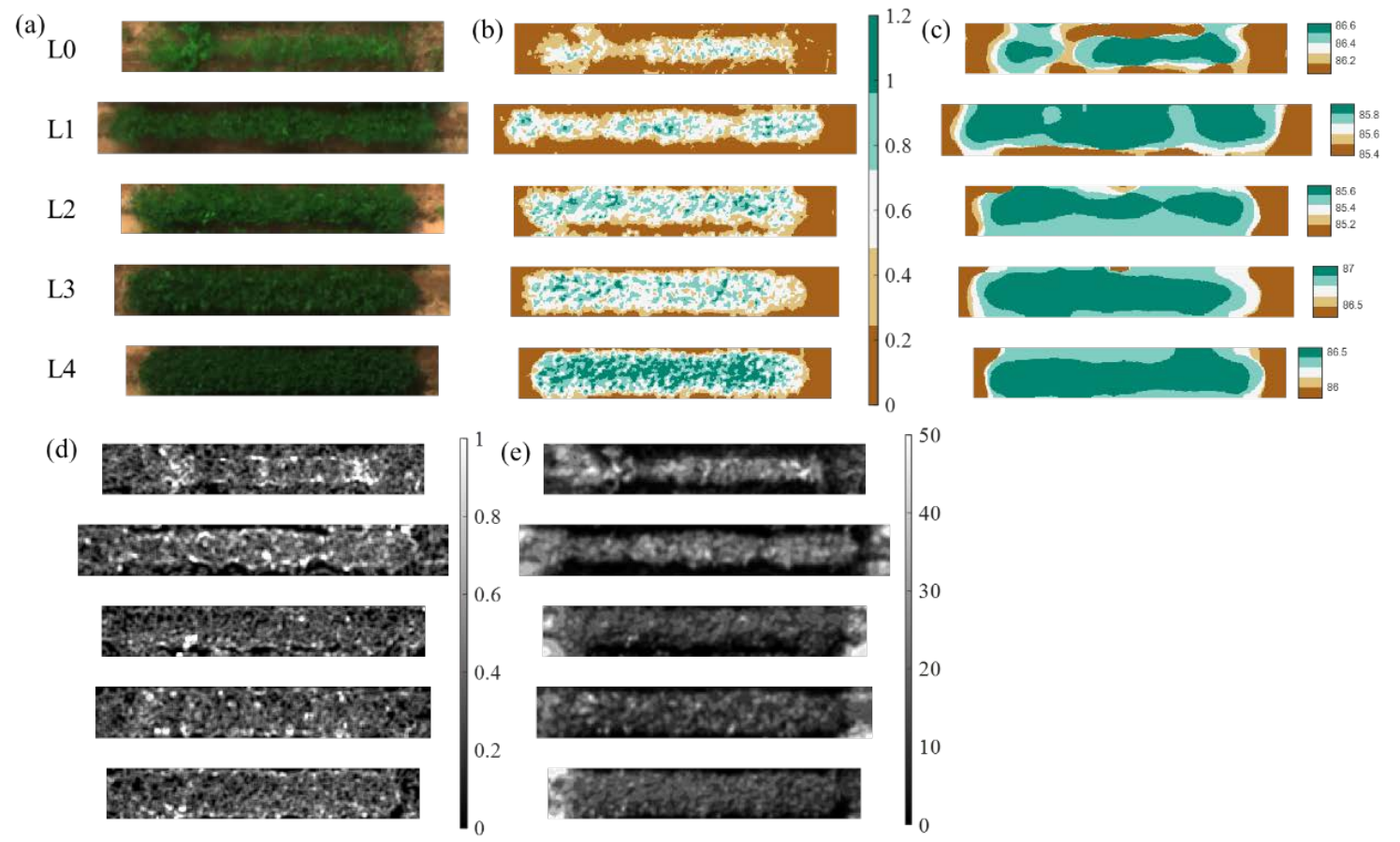

Figure 3-8. Images of soybean plots with yield in five levels. (a) - (e) are images in RGB, MTVI2, DEM, CVI_contrast, and CVI_entropy, respectively.

It is also obvious that the high-yield soybean plots, such as L4, had a full and wide canopy shape, while those with low yield, e.g. L0, were slim and discontinuous. In the DEM images in Figure 3-8c, the center part (green color) of L4 showed a complete runway shape and covered the majority of the image, while as yield decreasing, the center part starts shrinking and clustered. Compared to the images in VIs, a DEM image contains only depth information excluding color details and other noises, leading to a relatively clear contour of the soybean canopy.

Based on our observations, canopies of soybean plots with lower yield were less uniform than plots with a higher yield. For example, the soybean plot L0 shown in Figure 3-8 had an inconsistent canopy width which may be due to poor germination or missing plants. There were also two clusters of weeds near two ends of the plot, taking away nutrition and water from crop plants. In the L3 plot, some dots with a light green color 
might because that drought sensitive varieties showed canopy wilting symptoms causing the lower side to show up (Zhou et al., 2020).

Texture contrast can signify the amount of contrast in local regions of an image by exponentially increasing the difference between neighbor pixels (Hall-Beyer, 2017). The texture contrast images were able to highlight the non-uniform canopies, such as weed clusters in L0, light green dots in L1 to L3, which showed as bright dots as shown in Figure 3-8 d. The texture feature calculated using entropy is used to quantify the level of disorder in an image and larger entropy indicates greater disorder (Hall-Beyer, 2017). As shown in Figure 3-8 e, the L4 with uniform canopy had a low and dense fog texture image, while the texture values in $\mathrm{L} 0$ were much higher due to the variations in its canopy.

One outlier was identified in Figure 3-7 b and d. From the illustration in Figure 38, soybean plots with high yield are bulky, dark green and have a smooth canopy texture, while those with low yield are slim, light green and varied. Comparing the outlier with these soybean plots that have similar yield but were predicted with small residuals, the large residuals came from their canopy appearances that were different from the normal features in their peers. The outlier rarely had productions but had a wider canopy and darker color than L1. It is possibly because the soybean plot failed to gain seed weight due to severe drought stress during its seed filling stages (Zhou et al., 2020), even though with equivalent flowers, nodes and vigor to other fairly yielded plots.

\subsection{Conclusions}

The potential of estimating the yield of soybean breeding plots under drought conditions using UAV imagery and convolutional neural network was investigated in this study. A population of 972 soybean breeding plots in three maturity groups was planted 
under rainfed conditions to select soybean genotypes with improved traits of drought tolerance and yield potential. The visual wilting score was given to each plot by breeders to evaluate their drought tolerance and classify the soybean wilting traits (slow- and fastwilting). RGB and multispectral images were acquired at the late vegetation (V6), early reproductive (R1) and late reproductive growth stages (R6-R8). The correlations between the yield and the six image features extracted from the images collected at three stages were evaluated, and statistical significance was observed in the correlations. Seven image features representing plant height, canopy color and canopy texture were used to develop a mixed CNN model for yield estimation. The predictors included the seven image features and two categorical factors, i.e. maturity groups and drought tolerance. Results show that image features collected at the early and late reproductive growth stages were comparably promising in estimating soybean yield. The agreement of predicted yield and the measured yield was high with the $R^{2}$ up to 0.78 and a root mean square error of $391.0 \mathrm{~kg} \cdot \mathrm{ha}^{-1}$ (33.8 $\%$ to average yield), leading to the conclusion that the UAV imagery and deep learning methods are promising in estimating yield for soybean breeding purposes.

\section{References}

Araus, J. L., Kefauver, S. C., Zaman-Allah, M., Olsen, M. S., \& Cairns, J. E. (2018). Translating high-throughput phenotyping into genetic gain. Trends in Plant Science, 23(5), 451-466. https://doi.org/10.1016/j.tplants.2018.02.001

Ashapure, A., Oh, S., Marconi, T. G., Chang, A., Jung, J., Landivar, J., et al. (2019). Unmanned aerial system based tomato yield estimation using machine learning. Paper presented at the Proceedings of SPIE - The International Society for Optical Engineering. https://doi.org/10.1117/12.2519129 
Barnes, E., Clarke, T., Richards, S., Colaizzi, P., Haberland, J., Kostrzewski, M., et al. (2000). Coincident detection of crop water stress, nitrogen status and canopy density using ground based multispectral data. Paper presented at the Proceedings of the Fifth International Conference on Precision Agriculture, Bloomington, MN, USA.

BATS Center, B. a. S. (1995). Methods of Plant Breeding. Retrieved from https://www.bats.ch/bats/publikationen/1995-1_TA/2-methods.php/ (accessed June 12, 2020).

Carter Jr, T. E. (1999). Recent advances in breeding for drought and aluminum resistance in soybean. Paper presented at the Proceedings at the World Soybean Research Conference VI Chicago, IL, 1999.

Chan, T. F., \& Vese, L. A. (2001). Active contours without edges. IEEE Transactions on image processing, 10(2), 266-277. https://doi.org/10.1109/83.902291

Chu, T., Chen, R., Landivar, J. A., Maeda, M. M., Yang, C., \& Starek, M. J. (2016). Cotton growth modeling and assessment using unmanned aircraft system visualband imagery. Journal of Applied Remote Sensing, 10(3). https://doi.org/10.1117/1.JRS.10.036018

Corn Agronomy (2015). Soybean Growth and Development. Retrieved from http://corn.agronomy.wisc.edu/Crops/Soybean/L004.aspx/ (accessed June 12, 2020).

Desclaux, D., Huynh, T.-T., \& Roumet, P. (2000). Identification of soybean plant characteristics that indicate the timing of drought stress. Crop Science, 40(3), 716722. https://doi.org/10.2135/cropsci2000.403716x

Elavarasan, D., Vincent, D. R., Sharma, V., Zomaya, A. Y., \& Srinivasan, K. (2018). Forecasting yield by integrating agrarian factors and machine learning models: A survey. Computers and Electronics in Agriculture, 155, 257-282. https://doi.org/10.1016/j.compag.2018.10.024

Fehr, W. (1987). Principles of cultivar development. New York, NY: Macmillian. Feng, A., Zhang, M., Sudduth, K. A., Vories, E. D., \& Zhou, J. (2019). Cotton yield 
estimation from UAV-based plant height. Transactions of the ASABE, 62(2), 393404. https://doi.org/10.13031/trans.13067

Freedman, D., Pisani, R., \& Purves, R. (2007). Statistics (4th ed.): New York, NY: W. W. Norton \& Company.

Gago, J., Douthe, C., Coopman, R. E., Gallego, P. P., Ribas-Carbo, M., Flexas, J., et al. (2015). UAVs challenge to assess water stress for sustainable agriculture. Agricultural Water Management, 153, 9-19. https://doi.org/10.1016/j.agwat.2015.01.020

Geipel, J., Link, J., \& Claupein, W. (2014). Combined spectral and spatial modeling of corn yield based on aerial images and crop surface models acquired with an unmanned aircraft system. Remote Sensing, 6(11), 10335-10355. https://doi.org/10.3390/rs61110335

Gill, J. L. (1963). Effect of population size, selection intensity, linkage and non-additive variability upon genetic change in simulated populations. (Doctor of Philosophy), Iowa State University, Ames, Iowa. Retrieved from https://lib.dr.iastate.edu/rtd/2347

Gitelson, A., \& Merzlyak, M. N. (1996). Signature analysis of leaf reflectance spectra: algorithm development for remote sensing of chlorophyll. Journal of Plant Physiology, 148(3-4), 494-500. https://doi.org/10.1016/S0176-1617(96)80284-7

Goodfellow, I., Bengio, Y., \& Courville, A. (2016). Deep learning: Cambridge, MA: MIT press.

Haboudane, D., Miller, J. R., Pattey, E., Zarco-Tejada, P. J., \& Strachan, I. B. (2004). Hyperspectral vegetation indices and novel algorithms for predicting green LAI of crop canopies: Modeling and validation in the context of precision agriculture.

Remote Sensing of Environment, 90(3), 337-352. https://doi.org/10.1016/j.rse.2003.12.013

Hall-Beyer, M. (2017). GLCM texture: A tutorial. v.3.0. National Council on Geographic Information and Analysis Remote Sensing Core Curriculum, 3.

Herrmann, I., Bdolach, E., Montekyo, Y., Rachmilevitch, S., Townsend, P. A., \& 
Karnieli, A. (2019). Assessment of maize yield and phenology by drone-mounted superspectral camera. Precision Agriculture, 21, 51-64.

https://doi.org/10.1007/s11119-019-09659-5

Hincks, J. (2018). The World Is Headed for a Food Security Crisis. Here's How We Can Avert It. Retrieved from https://www.un.org/development/desa/en/news/population/world-populationprospects-2017.html/ (accessed June 12, 2020).

Hunt, E. R., Daughtry, C., Eitel, J. U., \& Long, D. S. (2011). Remote sensing leaf chlorophyll content using a visible band index. Agronomy Journal, 103(4), 10901099. https://doi.org/10.2134/agronj2010.0395

Ioffe, S., \& Szegedy, C. (2015). Batch normalization: Accelerating deep network training by reducing internal covariate shift. arXiv preprint. arXiv:1502.03167

James, G., Witten, D., Hastie, T., \& Tibshirani, R. (2013). An introduction to statistical learning (Vol. 112): Berlin, Germany: Springer.

Kanning, M., Kühling, I., Trautz, D., \& Jarmer, T. (2018). High-resolution UAV-based hyperspectral imagery for LAI and chlorophyll estimations from wheat for yield prediction. Remote Sensing, 10(12). https://doi.org/10.3390/rs10122000

Kron, A. P., Souza, G. M., \& Ribeiro, R. V. (2008). Water deficiency at different developmental stages of Glycine max can improve drought tolerance. Bragantia, 67(1), 43-49. https://doi.org/10.1590/S0006-87052008000100005

Krueger, S. (2019). How Breeders Develop New Soybean Varieties. Retrieved from https://emergence.fbn.com/inputs/how-breeders-develop-new-soybean-varieties/ (accessed June 12, 2020).

Kutner, M. H., Nachtsheim, C. J., Neter, J., \& Li, W. (2005). Applied linear statistical models (Vol. 5): New York, NY: McGraw-Hill.

LeCun, Y., Bengio, Y., \& Hinton, G. (2015). Deep learning. Nature, 521(7553), 436-444. https://doi.org/10.1038/nature14539

Liu, X., Zhang, K., Zhang, Z., Cao, Q., Lv, Z., Yuan, Z., et al. (2017). Canopy 
chlorophyll density based index for estimating nitrogen status and predicting grain yield in rice. Frontiers in Plant Science, 8(1829).

https://doi.org/10.3389/fpls.2017.01829

Maimaitijiang, M., Sagan, V., Sidike, P., Hartling, S., Esposito, F., \& Fritschi, F. B. (2020). Soybean yield prediction from UAV using multimodal data fusion and deep learning. Remote Sensing of Environment, 237, 111599.

https://doi.org/10.1016/j.rse.2019.111599

Maja, J. M. J., Campbell, T., Camargo Neto, J., \& Astillo, P. (2016). Predicting cotton yield of small field plots in a cotton breeding program using UAV imagery data. Paper presented at the Proceedings of SPIE - The International Society for Optical Engineering. https://doi.org/10.1117/12.2228929

Moghimi, A., Yang, C., \& Anderson, J. A. (2020). Aerial hyperspectral imagery and deep neural networks for high-throughput yield phenotyping in wheat. Computers and Electronics in Agriculture, 172, 105299. https://doi.org/10.1016/j.compag.2020.105299

Moreira, F. F., Hearst, A. A., Cherkauer, K. A., \& Rainey, K. M. (2019). Improving the efficiency of soybean breeding with high-throughput canopy phenotyping. Plant methods, 15(1), 139. https://doi.org/10.1186/s13007-019-0519-4

Passioura, J. (2007). The drought environment: physical, biological and agricultural perspectives. Journal of Experimental Botany, 58(2), 113-117. https://doi.org/10.1093/jxb/erl212

Pathan, S. M., Lee, J.-D., Sleper, D. A., Fritschi, F. B., Sharp, R. E., Carter Jr., T. E., et al. (2014). Two soybean plant introductions display slow leaf wilting and reduced yield loss under drought. Journal of Agronomy and Crop Science, 200(3), 231236. https://doi.org/doi:10.1111/jac.12053

Rosolem, C., Suzuki, S., Yuyama, M., \& Camacho, S. (2005). Papel do Brasil no combate a fome no mundo. Boletim de pesquisa da soja, (9), 95-102.

Rubel, F., Brugger, K., Haslinger, K., \& Auer, I. (2017). The climate of the European Alps: Shift of very high resolution Köppen-Geiger climate zones 1800-2100. 
Meteorologische Zeitschrift, 26(2), 115-125.

https://doi.org/10.1127/metz/2016/0816

Sadok, W., \& Sinclair, T. R. (2010). Transpiration response of 'slow-wilting' and commercial soybean (Glycine max (L.) Merr.) genotypes to three aquaporin inhibitors. Journal of Experimental Botany, 61(3), 821-829. https://doi.org/10.1093/jxb/erp350

Shipman, J. W. (2012). Introduction to colour theory. Retrieved from https://pdf4pro.com/view/introduction-to-colour-theory-new-mexico-institute-of4ac193.html/ (accessed June 12, 2020).

Singh, A. K., Ganapathysubramanian, B., Sarkar, S., \& Singh, A. (2018). Deep learning for plant stress phenotyping: trends and future perspectives. Trends in Plant Science, 23(10), 883-898. https://doi.org/10.1016/j.tplants.2018.07.004

Sleper, D. A., \& Poehlman, J. M. (2006). Breeding field crops. Hoboken, NJ: WileyBlackwell.

Smith, A. R. (1978). Colour gamut transform pairs. Comput Graph, 12(3), 12-19. https://doi.org/10.1145/965139.807361

USDA-FAS. (2020). World Agricultural Production. Retrieved from https://apps.fas.usda.gov/psdonline/circulars/production.pdf/ (accessed June 12, 2020).

Valliyodan, B., Ye, H., Song, L., Murphy, M., Nguyen, H. T., \& Shannon, J. G. (2016). Genetic diversity and genomic strategies for improving drought and waterlogging tolerance in soybeans. Journal of Experimental Botany, 68(8), 1835-1849. https://doi.org/10.1093/jxb/erw433

Vincini, M., Frazzi, E., \& D’Alessio, P. J. P. A. (2008). A broad-band leaf chlorophyll vegetation index at the canopy scale. 9(5), 303-319. https://doi.org/10.1007/s11119-008-9075-z

Westcott, P., \& Hansen, J. (2014). USDA Agricultural Projections to 2025. USDA Economic Research Service. 
Whitaker, R. T. (1998). A level-set approach to 3D reconstruction from range data. International journal of computer vision, 29(3), 203-231. https://doi.org/10.1023/A:1008036829907

Ye, H., Song, L., Schapaugh, W. T., Ali, M. L., Sinclair, T. R., Riar, M. K., et al. (2019). The importance of slow canopy wilting in drought tolerance in soybean. Journal of Experimental Botany, 71(2), 642-652. https://doi.org/10.1093/jxb/erz150

Yu, N., Li, L., Schmitz, N., Tian, L. F., Greenberg, J. A., \& Diers, B. W. (2016). Development of methods to improve soybean yield estimation and predict plant maturity with an unmanned aerial vehicle based platform. Remote Sensing of Environment, 187, 91-101. https://doi.org/10.1016/j.rse.2016.10.005

Zhang, X., Zhao, J., Yang, G., Liu, J., Cao, J., Li, C., et al. (2019). Establishment of plotyield prediction models in soybean breeding programs using UAV-based hyperspectral. Remote Sensing, 11(23), 2752. https://doi.org/10.3390/rs11232752

Zhou, J., Yungbluth, D., Vong, C. N., Scaboo, A., \& Zhou, J. J. R. S. (2019). Estimation of the maturity date of soybean breeding lines using UAV-based multispectral imagery. Remote Sensing, 11(18), 2075. https://doi.org/10.3390/rs11182075

Zhou, J., Zhou, J., Ye, H., Ali, M. L., Nguyen, H. T., \& Chen, P. (2020). Classification of soybean leaf wilting due to drought stress using UAV-based imagery. Computers and Electronics in Agriculture, 175, 105576.

https://doi.org/10.1016/j.compag.2020.105576 


\section{CHAPTER FOUR}

\section{VARIETY SELECTION USING UAV HIGH-THROUGHPUT PHENOTYPING TOWARDS IMPROVE SELECTION ACCURACY AND INTENSITY FOR SOYBEAN BREEDING}

\subsection{Abstract}

The efficiency of a crop breeding strategy is evaluated by genetic gain in the primary trait, i.e. yield, achieved in one year through artificial selection of advanced breeding materials. Selection for yield by measuring yield itself is a labor-intensive procedure, especially for progeny rows, and sometimes inaccurate due to its complex genetic behavior and high genotype-environment interaction. Indirect selection by considering crop secondary traits may improve genetic gain when the traits are less expensive but more accurate to measure than yield, or by accelerating decision-making steps and consequently decreasing the breeding cycle if they can be selected in earlier developmental stages or generations. There has been a number of applications of a secondary trait that can be precisely measured with a High-throughput phenotyping platform. However, the selection criteria have not been well established for crop breeding due to the lack of knowledge about the phenotypes responsible for the growth and development of superior genotypes. The goal of this study is to model the breeder's selection using a group of agronomic traits and investigate the potential of selecting superior soybean varieties for soybean breeding programs using image-based secondary traits. A total of 11473 progeny rows were planted in 2018 (PT) and 1773 among them were selected for the preliminary yield trial (PYT) in 2019 and 238 were then selected for the advance yield trial (AYT) in 2020. Seven agronomic traits, including yield, plant height, maturity data, flower and pubescence color, moisture and lodging were manually 
measured for soybeans in the two yield trials. Unmanned Aerial Vehicle-based images were collected every two weeks over the growing seasons, and a group of image features was extracted from five-band multispectral images for each trial. Results show that yield is the primary trait for selecting superior soybean lines as there were significant differences in yield between the selected and non-selected group for both yield trials. Profile analysis on the image features showed that progeny rows had the most variation among three trials and the images collected at earlier stages (before R5) explained more variation than those at later stages. The lasso model for selecting soybean lines with image features correctly identified $71 \%$ and $76 \%$ of the breeder's selection for the PT and PYT and the model's selections were shown to have a significantly higher yield than the breeder's selection regarding yield in the following year's trial. The model selections in PT and PYT had respectively $4 \%$ and $5 \%$ higher yield, comparing the breeder's selection. It could be concluded that the proposed model is promising in making selections on soybean breeding trials.

Keywords: high-throughput phenotyping; soybean breeding; machine learning; profile analysis; variety selection

\subsection{Introduction}

Crop breeding is the art and science of changing the traits of crops in order to produce desired characteristics (Sleper \& Poehlman, 2006). From 10,000 years ago, plants with preferrable phenotypes have been selected and bred by human for higher productions and environmental adaptivity (Ahmar et al., 2020). Since the early 1900s, crop development has been systemized with the introduction of Mendelian laws, followed by cross-breeding and hybrid breeding that are referred to as conventional breeding strategies. 
It usually takes one or two decades to create a new cultivar by conventional breeding, given the many phases of crossing, selection, and testing involved in the production of new plant varieties, (Ahmar et al., 2020). With the availability of high-throughput genome information, molecular breeding strategies were developed to shorten conventional breeding cycles with the aid of genetic markers (Xu et al., 2017). For example, markerassisted selection (MAS) and genomic selection (GS) rely on marker-trait associations and are both routinely applied in modern plant breeding with the objective of selecting individuals with superior performance (Arruda et al., 2016).

Despite genomics and technical advances, identifying plants with desirable traits is the initial and most important step in breeding pipelines (Ahmar et al., 2020). It is well acknowledged in the breeding community that phenotyping efficiency still represents a bottleneck in crop breeding (Araus \& Cairns, 2014; Araus et al., 2018; Furbank \& Tester, 2011), as phenotypes are the bases to make selections in conventional breeding and a lack of suitable phenotyping data has led to poor results in gene/quantitative trait locus (QTL) discovery, limiting progress in genomics-assisted crop improvement programs (Mammadov et al., 2012).

The goal of crop breeding is to improve genetic gain that is the amount of increase in performance of a population achieved in one year through artificial selection (Fehr, 1991). Genetic gain can be increased by increasing population size (or selecting fewer numbers of varieties) to increase selection intensity, shortening the breeding cycle, ensuring suitable genetic variation in the population, and obtaining accurate estimates of the genetic values (Araus et al., 2018; Moreira et al., 2019; Xu et al., 2017). Among them, the selection intensity (or population size) is limited by the phenotyping capacity on 
measuring key agronomic traits of breeding materials and selection accuracy is limited by the lack of objective and efficient phenotyping tools.

In conventional breeding, there are several procedures following the timeline (as shown in Figure 1-1) to combine desired crop traits into individual crops and eventually select superior varieties. After identifying a key problem in a specific program, breeders select from parent lines from given populations that have desired traits to potentially address the problem. Then the selected parent lines are combined to obtain crosses that probably would exhibit simultaneously desired traits from both parents. The cross lines are advanced for a couple of years to obtain enough seeds for field evaluations. Normally 10,000 to 30,000 cross lines are made at the beginning of soybean breeding programs, depending on the scale of the programs.

In the first year of the field evaluations, the cross lines are planted in progeny trials (PTs). With the large number of lines and limited numbers of seeds for each entry, progeny lines are planted in one-row plots without replicates (Orf et al., 2004). Manual phenotyping of all such one-row plots in PTs requires a large investment of resources but is inaccurate due to the boundary effects of single rows (Moreira et al., 2019). As an alternative, the plots in PTs are visually examined by experienced breeders and a certain percentage of them (normally 10-15\%) will be selected for a combination of the desired traits eliminating those inheriting undesired traits from parents.

The selected progeny lines are kept planting in multi-location yield trials (YTs). In YTs, soybean lines are planted in four-row plots with two center rows as effective rows while two side rows as protection rows. Key traits, such as yield, plant height and maturity group, etc., are manually measured for each plot in YTs. This procedure will be repeated 
in many locations for 2-3 years. Breeders could keep selecting a certain percentage of lines (normally $10 \%$ each year) that are adapted either widely across the regions or specifically for targeted regions, depending on the purpose of the breeding program.

High-throughput phenotyping (HTP) has been demonstrated the ability to estimate various agronomic traits for different crops for breeding purposes, such as yield (J. Zhou, J. Zhou, et al., 2021), height (Watanabe et al., 2017), the maturity date (Zhou et al., 2019), detecting crop components (Makanza et al., 2018; Zhang et al., 2018) and stress symptoms (J. Zhou, H. Mou, et al., 2021; Zhou et al., 2020). With higher accuracy and efficiency, the estimates by HTP could be used as secondary traits additionally to yield to select a small group of superior lines, thus increasing the population size and selection intensity (Richards, 2000). Those that could be estimated at earlier growth stages or generations would accelerate the selection procedure and eventually shorten the breeding circles (Moreira et al., 2019).

Motivated by the need to improves the selection accuracy and intensity for soybean breeding, the goal of this study was to investigate the potential of selecting superior soybean progeny using the unmanned aerial vehicle (UAV)-based image features. There were three objectives to achieve this goal: (1) modeling breeder's selection (selected/nonselected) using manually measured crop traits (flower color, pubescence color, height, lodging, maturity date, moisture and yield) to quantify weights of the traits on the final selections, (2) evaluate the difference in image features between selected and non-selected genotypes, (3) identify the growth stages when the image features are effective in making selections by profiling the time-series image features, and (4) evaluate the performance of model selections by comparing their yield performances with breeder's selection in the 
following YTs.

\subsection{Material and Method}

\subsubsection{Plant material and field experiment}

A three-year breeding trial was conducted at three locations in Missouri, United States from 2018-2020, including a progeny trial (PT), a preliminary yield trial (PYT) in 2019 and an advanced yield trial (AYT) in 2020. The PT with 11473 soybean progeny rows derived from F4 plants was conducted in a 3.64 ha field at the Greenley Research Center of the University of Missouri (MU), Novelty, Knox County, Missouri. The progeny rows were planted without replicates in one-row plots of size $2.59 \mathrm{~m}$ with $0.76 \mathrm{~m}$ row spacing and planted on May 29, 2018.

A group of 1860 lines was selected from the PT among which 1773 were advanced as a PYT in a 4.09 ha field at the Bradford Research Center of MU, Columbia, Boone County, Missouri. The PYT lines were planted without replicates in four-row plots on June 3, 2019. A group of 238 lines selected from the PYT was evaluated in an AYT in a 1.76 ha field at Missouri Soybean Association, Columbia, Boone County, Missouri. The lines were planted with two replicates in four-row plots on June 2, 2020. Soybean plots in the PYT and AYT were in the same size and row spacing as those in the PT. In each of the three trials, replicated checks in MG III and IV were planted over the field as maturity references. 


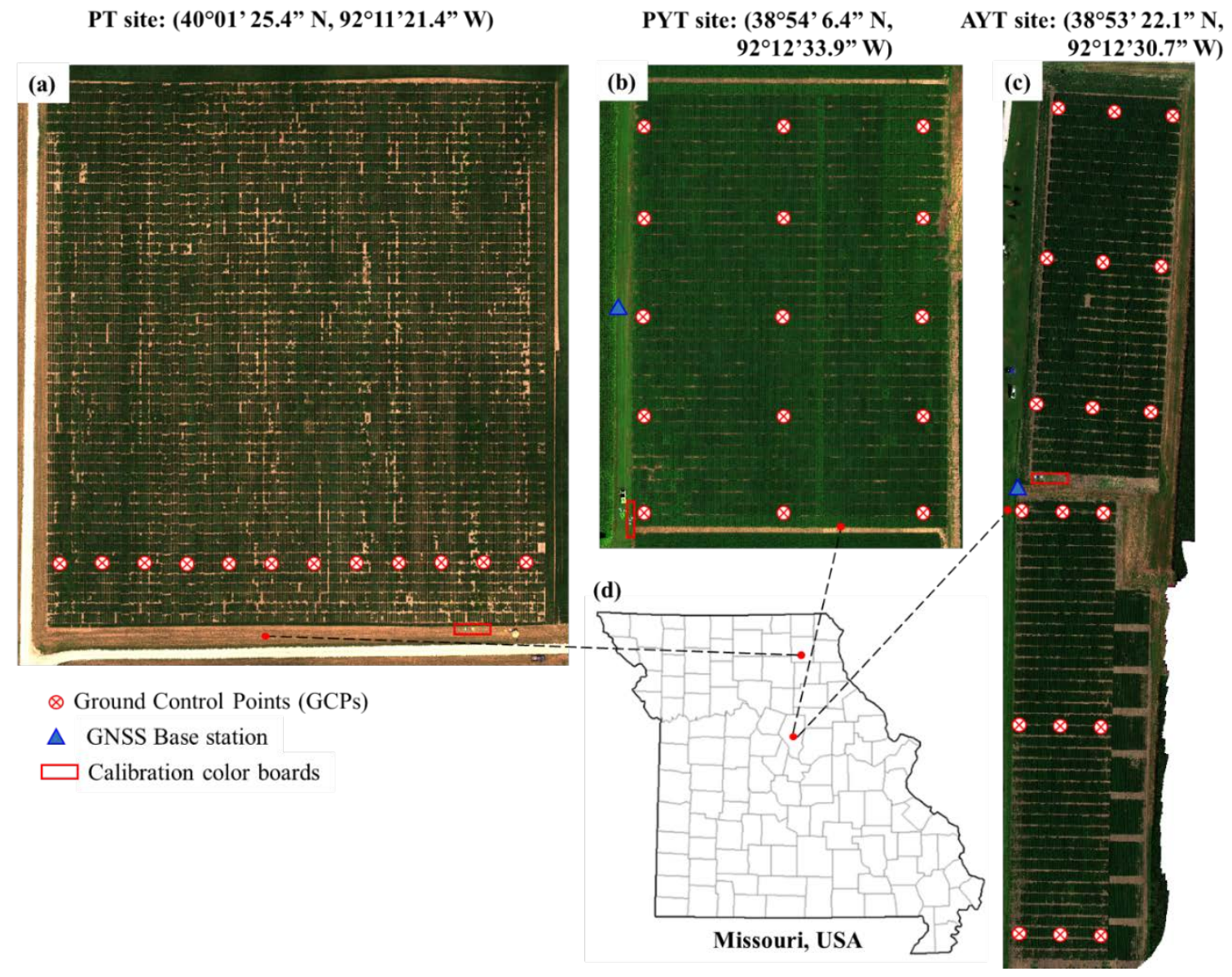

Figure 4-1. Illustration of the experimental fields. (a) The progeny trial in 2018. (b) The preliminary yield trial in 2019. (c) The advanced yield trial in 2020. (d) Locations of the fields.

\subsubsection{Manually measured agronomic traits}

Seven agronomic traits were manually measured for the four-row soybean plots in the PYT and AYT, including flower color, pubescence color, plant height, lodging, maturity date, seed moisture and grain yield. There were three flower colors noticed, including purple, white, and segregating (mixed colors) and four pubescence colors, including tawny, gray and segregating in the PYT and tawny, light tawny and gray in the AYT. Plant height was measured from the ground level to the leaf base of the highest fully expanded leaf by holding a measuring tape close to the stem of soybean lines vertically. Lodging is visually rated on a scale from $1-5$ with $1=$ plants standing upright while $5=$ 
plants prostrating on the ground. Maturity dates were visually determined and recorded as the number of days after September $1^{\text {st }}$ (day 1) in each of 2019 and 2020. A soybean plot was not considered mature until approximately 95\% of its pods achieved mature pod color (Zhou et al., 2019). Grain yield and seed moisture were measured during harvest in realtime with a combine.

\subsubsection{UAV data collection}

Imagery data were acquired using a UAV imaging system consisting of a UAV platform (model: DJI Matrice 600 Pro, DJI, Shenzhen, Guangdong, China) and a fivechannel multispectral (Blue-Green-Red-RedEdge-NIR) camera (RedEdge-M, MicaSense, Seattle, WA, USA) camera. The multispectral camera has a resolution of $1260 \times 960$ pixels and was configured to take time-lapse images at $1 \mathrm{fps}$ (frame per second) and calibrated using a spectral standard board before flights following the procedure described in (Zhou et al., 2019). The multispectral camera has a Global Navigation Satellite System (GNSS) receiver unit embedded and pre-programmed to provide geo-referencing information. The information was stored in EXIF (Exchangeable Image File Format) metadata associated with each frame of the images. Before each flight, a calibration reflectance panel (CRP) was imaged by holding the camera at about $1 \mathrm{~m}$ above the CRP and looking vertically in an open area (to avoid shadow).

Data were collected on four times (75, 90, 108 and 121 day after planting (DAP)) in the 2018 PT, six times (45, 57, 74, 87, 109 and 120 DAP) in the 2019 PYT and six times (51, 64, 78, 94, 107 and 120 DAP) in the 2020 AYT. The UAV imaging system flew at 30 $\mathrm{m}$ above ground level at a nadir view for all data collections, resulting in ground sampling distances (GSDs) of $20.8 \mathrm{~mm} \cdot$ pixel $^{-1}$ for the multispectral images. Before each flight, the 
flight speed was set to $7 \mathrm{~km} \cdot \mathrm{h}^{-1}$ and the flight paths were designed to ensure a forward overlap of $\geq 70 \%$ and side overlap of $\geq 65 \%$ for all images using a flight control App Autopilot (Hangar Technology, Austin, TX, USA).

Twelve, 15 and 18 ground control points (GCPs) were placed evenly for each data collection in the PT, PYT and AYT field, respectively. The GCP was made of a $30 \times 30 \mathrm{~cm}$ wood square covered with a cross-patterned vinyl sheet that was mounted on the top of a $1.1 \mathrm{~m}$ plastic fence post. The GCPs were manually stepped into soil before each data collection, and each had a height of $99 \mathrm{~cm}$ above the ground. The GNSS coordinates of the GCPs were obtained using a Reach Real-Time Kinematic (RTK) GNSS receiver system (Reach RS+, Emlid, St. Petersburg, Russia). The system consists of a base station and a rover receiver. The base station was mounted on a tribrach that was fixed in an open area in the fields to ensure sufficient satellite reception for each data collection. As accurate absolute GNSS accuracy is not required for this study, but precise relative accuracy of the rover to the base station, the base position with an averaged single accuracy ( $\sim 2.5 \mathrm{~m})$ was obtained for each field by accumulating its GNSS coordinates for 5 min (Emlid, 2021). The base station was maintained stationary and horizontally during every flight and its position was fixed over a growing season. The rover receiver was mounted on a monopod that was placed vertically in the holes after the GCPs were pulled out. The GNSS coordinate of each GCP was recorded after accumulating for 10 sec using ReachView that is a manufacturer-developed App for users to take full control of the Reach modules.

\subsubsection{Image processing}

The images were processed using a semi-automatic pipeline to generate numeric inputs for data analysis and modeling. The pipeline mainly includes (1) Generating 
Orthomosaic images; (2) Plot separation and (3) Feature calculation.

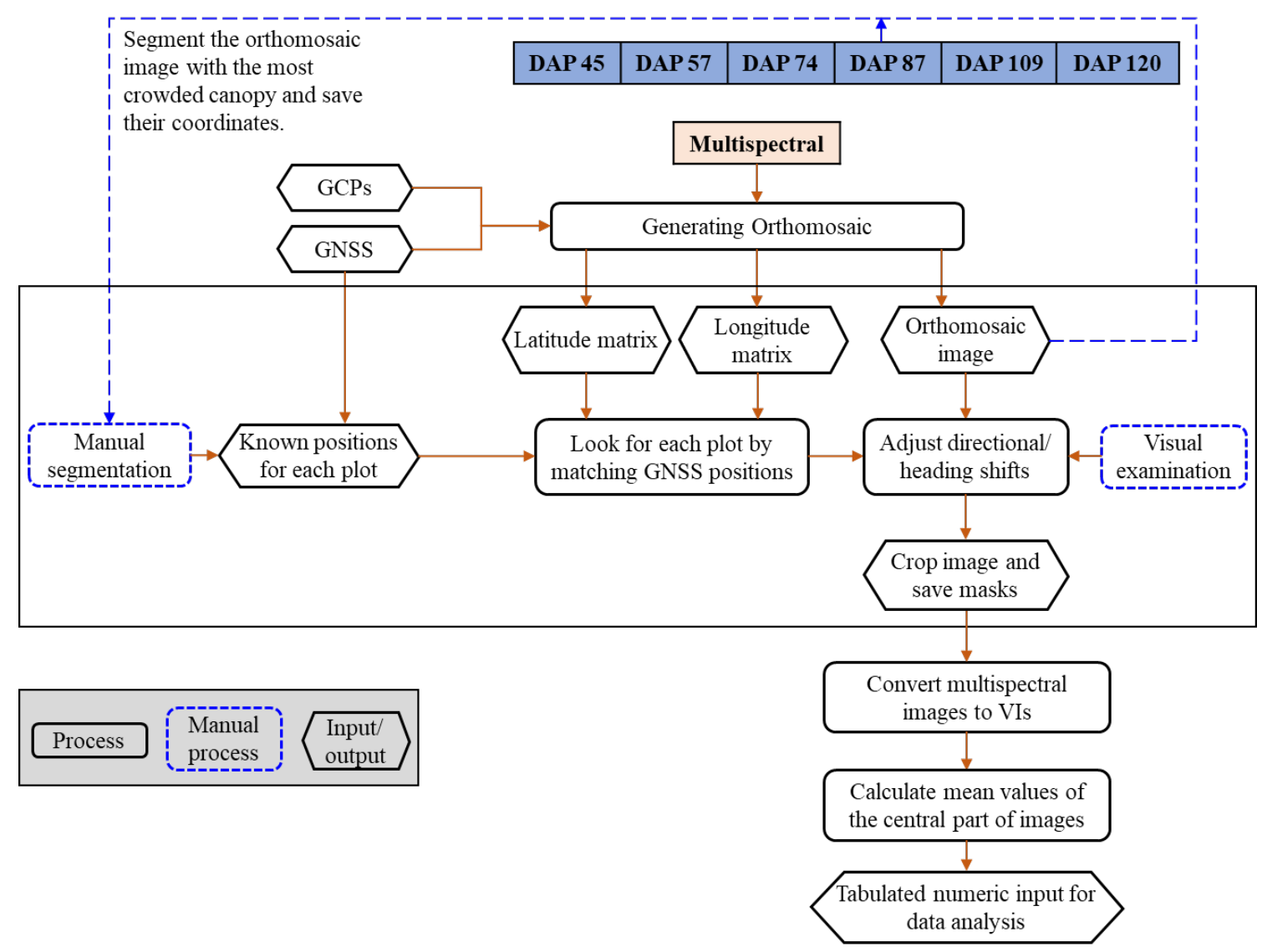

Figure 4-2. The image processing pipeline

The multispectral images were processed using Pix4D Mapper (Pix4D, Lausanne, Switzerland) to generate orthomosaic images. After all the images were imported, the georeferencing information was read automatically from the EXIF metadata. The images of the CRP were manually cropped for reflectance calibration (Zhou et al., 2019). The generated orthomosaic images and DEMs were then exported as the tag image file format (tiff) that allows GNSS position tagged with each pixel being exported together. The exported images were then processed using the Mapping Toolbox and Image Processing Toolbox of MATLAB (Version 2019a, The MathWorks, Natick, MA, USA).

GNSS positions (i.e., Latitude and Longitude) are stored in the exported 
orthomosaic images as two matrices that have the same dimensions as the images. One matrix has only latitude values and the other has only longitude. The coordinates were read using the 'pixcenters' function with a 'makegrid' option in the Mapping toolbox in MATLAB. The projected geolocation coordinate system of the multispectral images was converted into the World Geodetic System 1984 (WGS84) using the function 'projinv' with required information read from the orthomosaic images.

Individual four-row plots were manually separated from the orthomosaic image when soybean plants had the bulkiest canopy. A rectangle mask was given to each plot and then visually examined to ensure the plot was separated correctly regarding its positions and boundaries. The mask was applied to the coordinate matrices to obtain latitude and longitude values of the plot. New rectangle masks were generated by looking for pixel positions of the previous latitude and longitude values of the plot from the coordinate matrices of other orthomosaic images and then applied to separate the plot from those images. Directional and heading shifts might occur when matching coordinates for different images due to GPS and stitching errors. The shifts were identified by visual examination and measured by calculating the difference between the matched coordinates and the manual adjustments of the masks at four corners. The saved coordinate values were applied to separate plots from the images on other days with the same procedure for adjusting shifts.

The separated images containing a single-row soybean were used to calculate image features. The center part of each row was cropped by removing the image edge (a quarter of image width on each width side and a quarter of image length on each length side). Thirty-eight image features were calculated from the five-band images, including the band 
of green and red edge, as well as 36 vegetation indices listed in Appendix A.

\subsubsection{Model training and validation}

Soybean plots in the PT and PYT were randomly split into training (80\%, i.e., 9178 and 1418 plots for the PT and PYT, respectively) and testing (20\%, 2295 and 790 plots for the PT and PYT, respectively) datasets to train and validate the variety selection model. Two Lasso (Least absolute shrinkage and selection operator) models were trained separately for the PT and PYT. The model was built using the 'cv.glmnet' function in the 'glmnet' $\mathrm{R}$ package by specifying the model type argument alpha $=1$. The shrinkage parameter lambda was tuned in the model regarding test errors by 5 -fold cross-validation. The trained models were applied on the test sets to predict if the plots were selected (1) or non-selected (0). The model output for each plot was a probability $(p)$ of it being selected or non-selected. The plot was classified to the selected group if $p>0.5$, or the non-selected group, otherwise.

The model performance was evaluated by the classification recall (Eq. 4-1) of the testing sets. As shown in Figure 4-3, the true positive (TP) class represents the overlapping between the model selections and breeder's selection, while the false negative (FN) class contains those who were not selected by the model but selected by the breeder. In this study, the FN class needs to be avoided, i.e., the minimum type 2 error. Recall measures the proportion of the TP class out of all the true classes. In breeding programs, it is always preferred that the selected genotypes have the increased yield from one generation to the next (Moreira et al., 2019). To evaluate the model performance, yield means in the following yield trials of the TP class (the model selections) were compared to the yield means of the FN class (the model non-selected group but selected by breeders). 


$$
\text { Recall }=\frac{T P}{T P+F N}
$$

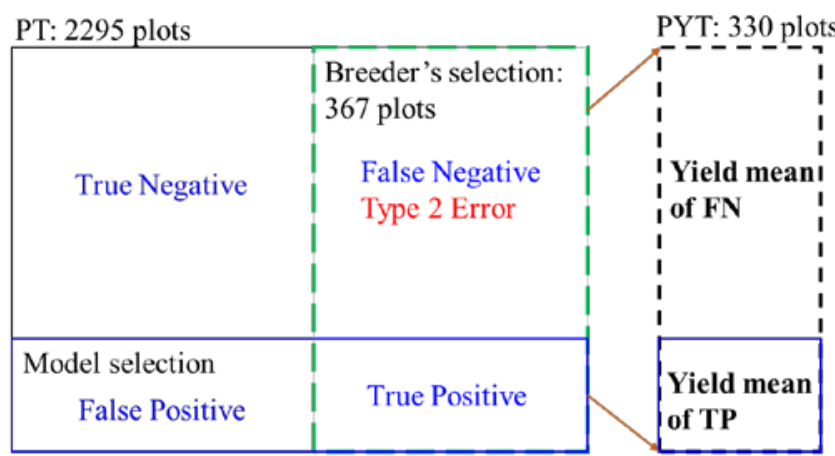

Figure 4-3. Illustration of two metrics for evaluating model performance for selecting superior soybean plots

\subsubsection{Data analysis}

Data analyses were conducted using the R language in RStudio (Ver. 1.3.1093, RStudio, Boston, MA, USA). A one-way analysis of variance (ANOVA) with an honest significant difference (HSD) Tukey test was conducted to evaluate the differences in manually measured agronomic traits between the selected and non-selected soybean varieties. Principal component analysis (PCA) was performed to estimate the relationship among the manually measured agronomic traits and model the breeder's selection criterion.

A multivariate ANOVA (MANOVA) was conducted to test the null hypothesis that no difference in image features averagely for multiple data collections between the two groups. Profile analysis was conducted to evaluate the time behavior of image features for the selected and non-selected groups. The null hypothesis of the profile tests is defined as:

$$
H_{0}: \boldsymbol{L B} \boldsymbol{M}=\mathbf{0}
$$

where $\boldsymbol{L}=[1,-1], \boldsymbol{B}=\left[\begin{array}{lll}\mu_{11} & \mu_{12} & \mu_{1 p} \\ \mu_{21} & \mu_{22} & \mu_{2 p}\end{array}\right]$, and $\boldsymbol{M}=\left[\begin{array}{cccc}1 & 0 & \ldots & 0 \\ -1 & 1 & \cdots & 0 \\ \vdots & & \ddots & \vdots \\ 0 & 0 & \cdots & -1\end{array}\right] . \boldsymbol{L}$ is the indictor matrix of comparisons between the selected and non-selected groups. $\boldsymbol{B}$ is the matrix of the mean responses in an image feature between the two groups over time, and $p$ 
represents the times of data collection in each trial. In this study, $p=4,7$ and 7 for the PT, PYT and AYT, respectively. $\boldsymbol{M}$ is the matrix of comparisons across time. The profile analysis was performed using the 'linearHypothesis' function in the 'car' package (S. Zhou et al., 2021).

\subsection{Results}

4.4.1 Differences in the manually measured agronomic traits between the selected and unselected groups

Figure 4-4 shows the result of one-way ANOVA tests on the manually measured agronomic traits that were collected in the yield trials. In the PYT, significant differences were observed in yield between the two groups, with the selected group averagely producing $2804.2 \mathrm{~g}$ seed weight and the non-selected group $2427.1 \mathrm{~g}$ (Figure 4-4 a). The yield difference was observed consistently in the AYT, with the selected group averagely producing $2605.1 \mathrm{~g}$ seed weight and the non-selected group 2330.7g (Figure 4-4 a). In the PYT, plant height had a dynamic range from 45 to $125 \mathrm{~cm}$ and the selected group (89.6 $\mathrm{cm})$ was significantly taller than the non-selected one $(87.9 \mathrm{~cm})$, while in the AYT there was no difference in plant height $(55-105 \mathrm{~cm})$ between the two groups. No significant differences were observed between the two groups in lodging and moisture for the PYT. Similarly, there is no significant difference in moisture for the AYT. It is noted that the selected group had higher lodging readings than the non-selected group, which were possibly caused by the fewer variations in lodging, as most of the plots in the AYT had the lodging readings within the range of $1-2$, except for a few plots over 3 . For the maturity date, the selected groups were mostly located between 25-37 and 30-37 days after August $1^{\text {st }}$. From Figure 4-4 f, g and 4-5f, g, flower pubescence colors were not the decisive factors 
because certain percentages of selection were made from each of the colors. However, it is obvious that color segregating is an indicator of the non-selection groups.
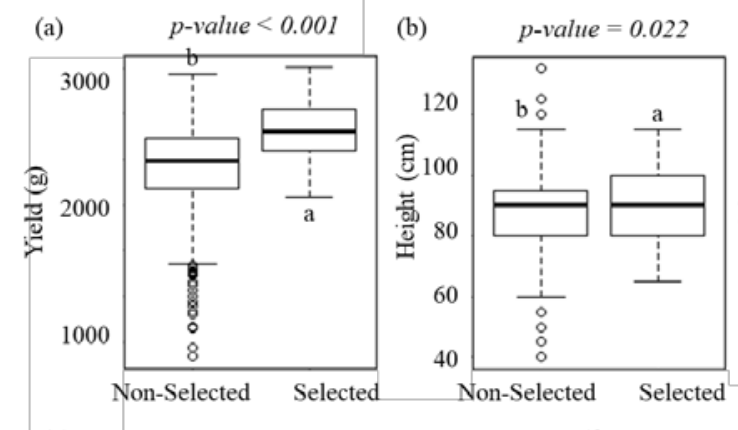

(c)
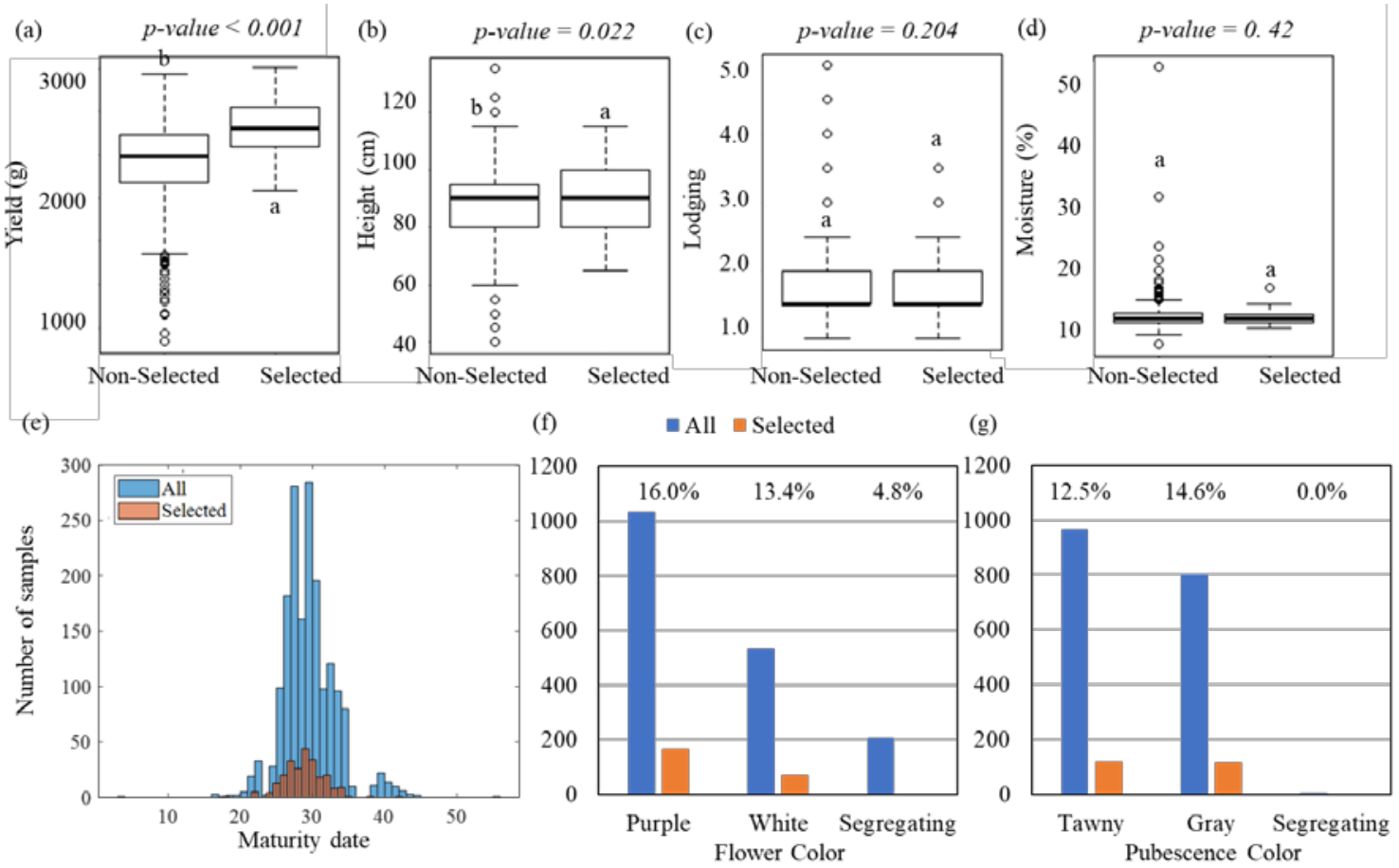

Figure 4-4. Comparisons of manually measured agronomic traits between the selected and nonselected groups in the PYT. (a) - (d) Boxplots of the comparisons in yield, height, lodging and moisture, respectively. The lower-case letters above bars indicate the significant difference among these means at the above significance levels (p-values). (e) Histogram of the maturity dates. (f) and (g) Bar plots of the flower and pubescence colors. For (e)-(g), the blue bars represent all plots, while the orange bars represent the selected plots. 

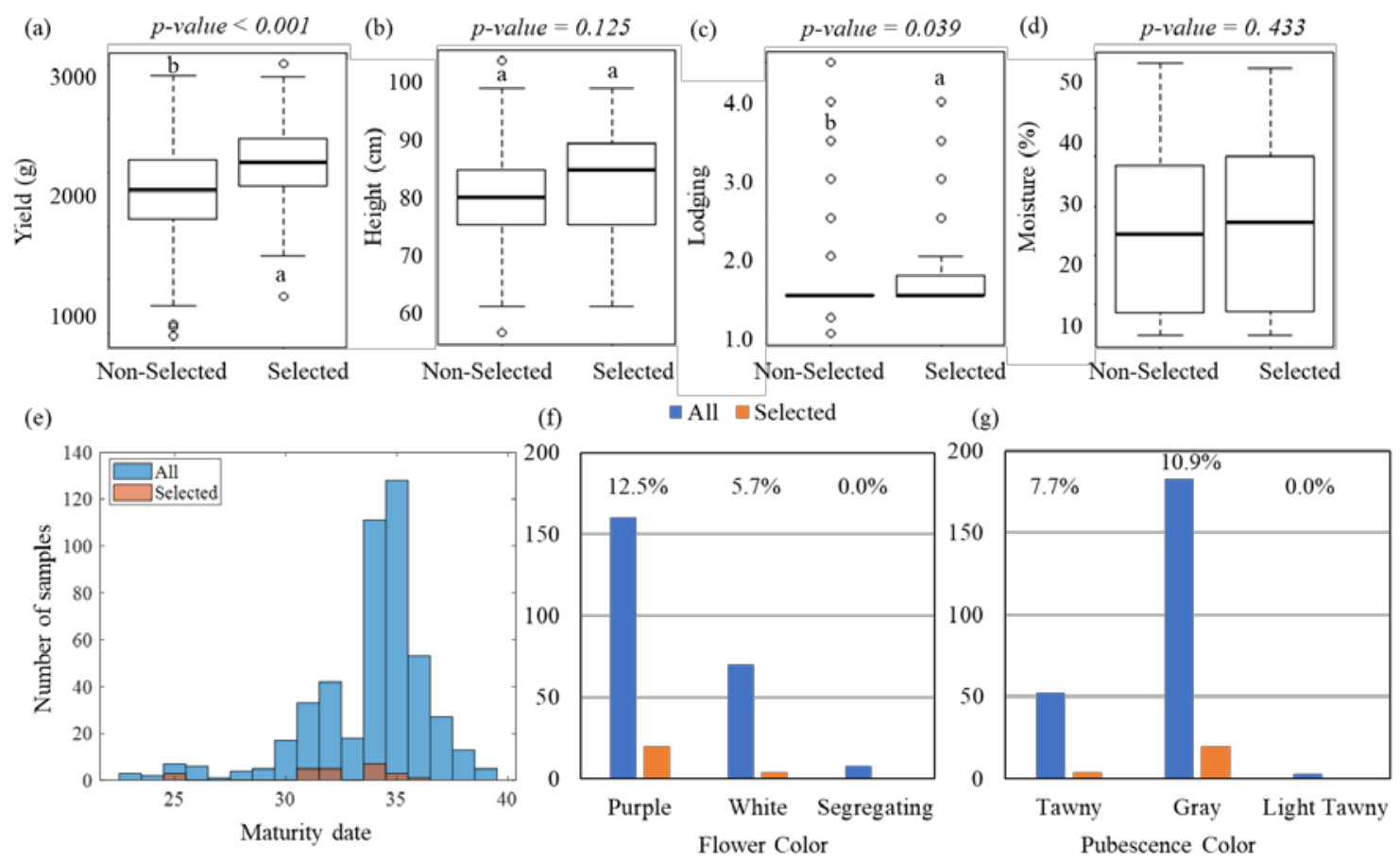

Figure 4-5. Comparisons of manually measured agronomic traits between the selected and nonselected groups in the AYT. (a) - (d) Boxplots of the comparisons in yield, height, lodging and moisture, respectively. The lower-case letters above bars indicate the significant difference among these means at the above significance levels (p-values). (e) Histogram of the maturity dates. (f) and (g) Bar plots of the flower and pubescence colors. For (e)-(g), the blue bars represent all plots, while the orange bars represent the selected plots.

\subsubsection{Modelling breeder’s selection criterion}

The PCA analytical results on the manually measured agronomic traits for the PYT and AYT are shown in Figure 4-6a and 4-7a. The PCA revealed three principal components (PCs) with eigenvalues $>1$ capturing $65.3 \%$ of the total variation for the PYT and three PCs capturing $62.4 \%$ for the AYT. In both yield trials, PC1 was primarily influenced by yield, height and maturity date. While PC2 was greatly influenced by moisture and lodging in PYT, and by maturity date and flower color in AYT. Yield and height had the highest contributions with consistent directions to PC1 for both trials. Moreover, it was noted from Figure 4-6c and 4-7c that height was significantly correlated with yield with positive linear coefficients. Lodging, maturity date and moisture contributed secondly. No consistent 
linear relationship was found between lodging and yield, as well as between the maturity date and yield over two years. Flower and pubescence color had very little contribution to the selection and no particular color was observed to have a higher yield than others. In PYT, soybean plots with the white flower color had a higher yield than those with purple and segregating, while those with the pure pubescence colors had a higher yield than segregating lines. In AYT, however, no significant difference was found among different colors. The relationship between yield and other agronomic traits shown in Figure 4-6 and 4-7 generally confirms the variable contributions found in the first two PCs in the PYT and AYT.

From the scatterplots of sample distribution based on PCA regression scores, it is obvious that the selected groups were mainly in the second and third quadrant, negatively directing to the axis of PC1. However, the selected group highly overlapped with the nonselected group in the first two PCs in both trials, and in each trial, the two PCs collectively accounted for less than $50 \%$ of the variations. 

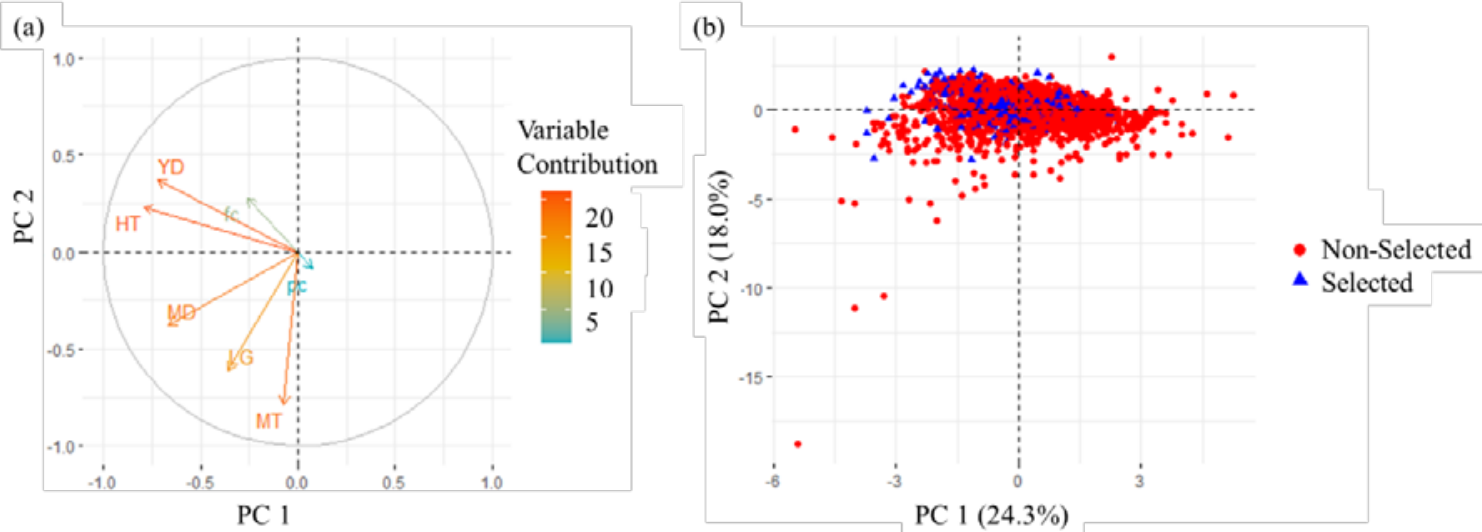

(c) Slope $=19.2, p$-value $<0.001$

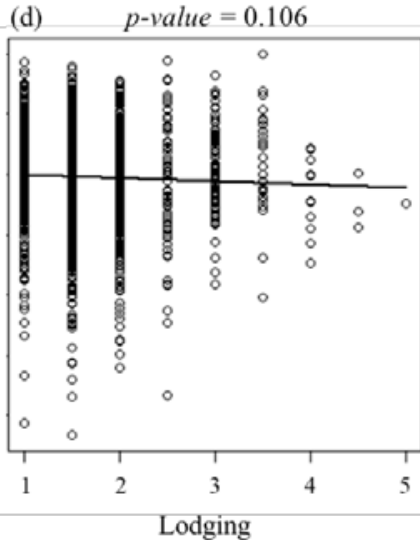

(e) Slope $=30.0, p$-value $<0.001$
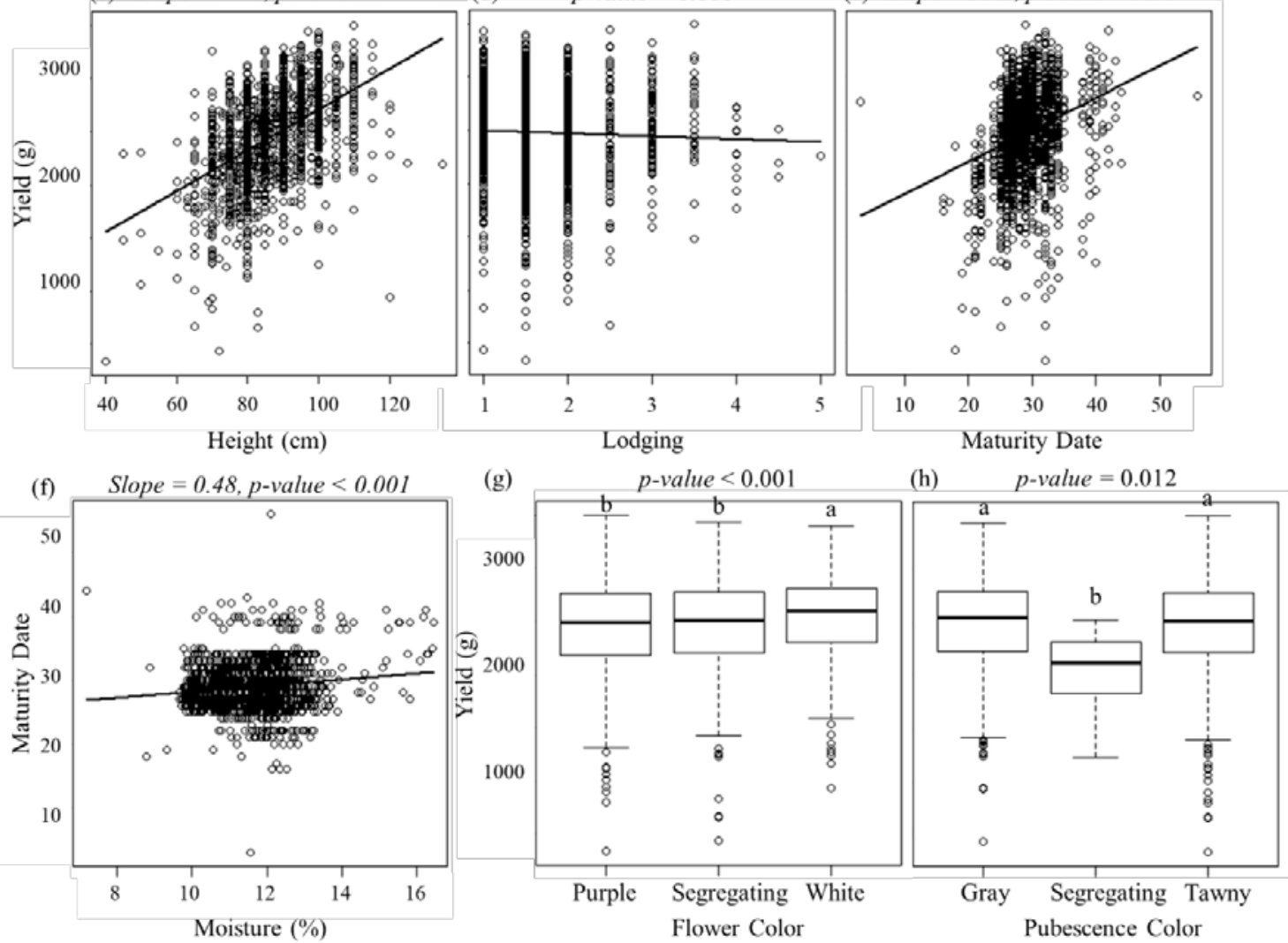

Figure 4-6. Manually measured agronomic traits in PYT. (a) are the visualization plots of the variables' contributions and directions in the plane of the first two PCs. (b) is the projection of sample variables on the first two PCs. (c)-(d) are scatterplots of yield versus height, lodging and maturity date, respectively. (h) is a scatterplot of the relationship between moisture and maturity date. Eight outliers with moisture values higher than $20 \%$ were removed to ensure the adequacy of linear regression. (g)-(h) are boxplots of the yield of three groups of flower color and three groups of pubescence color. The p-value above (c)-(h) indicates the significance level of estimation. 

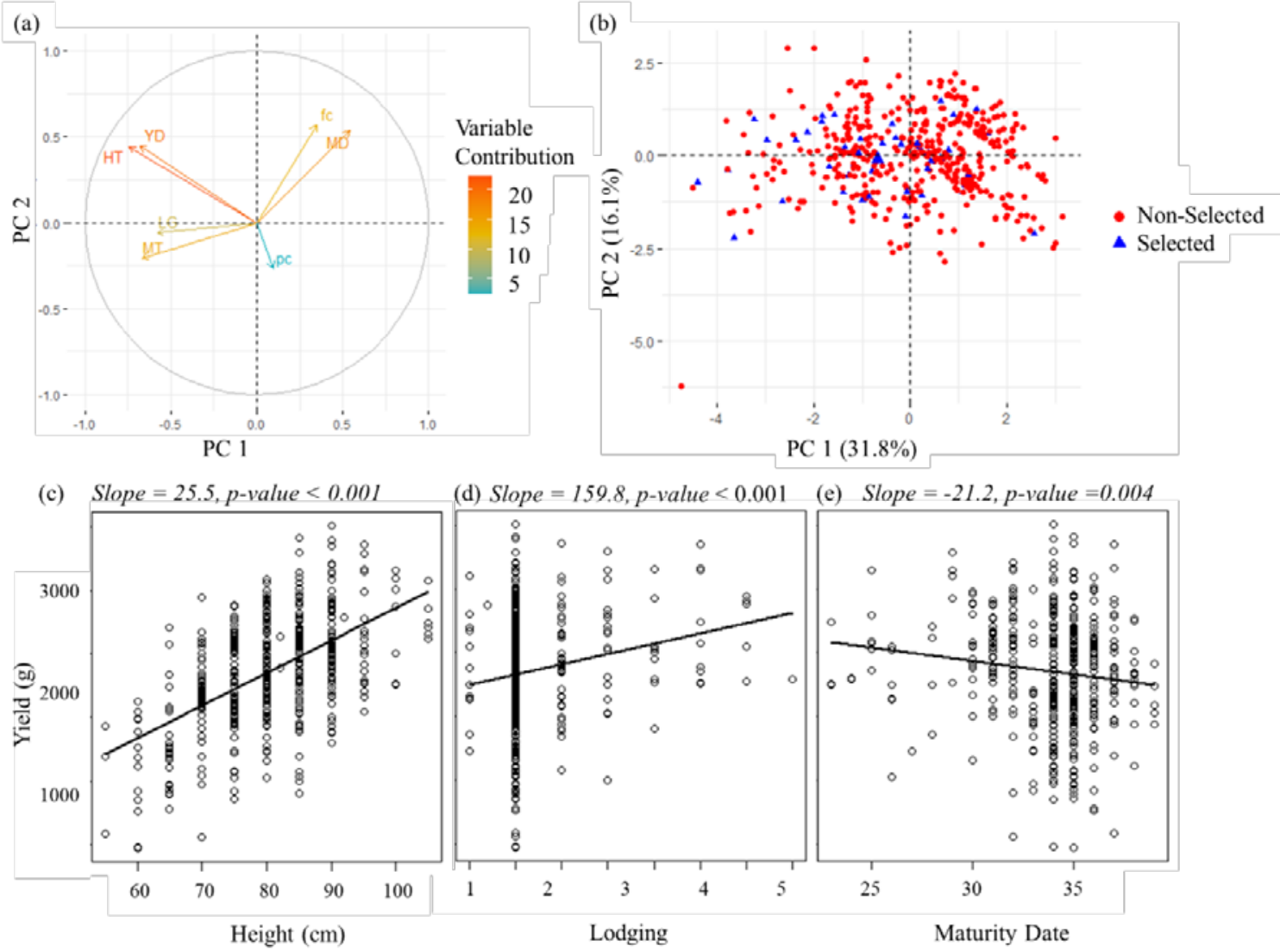

(d) Slope $=159.8$, p-value $<0.001 \quad$ (e) Slope $=-21.2, p$-value $=0.004$
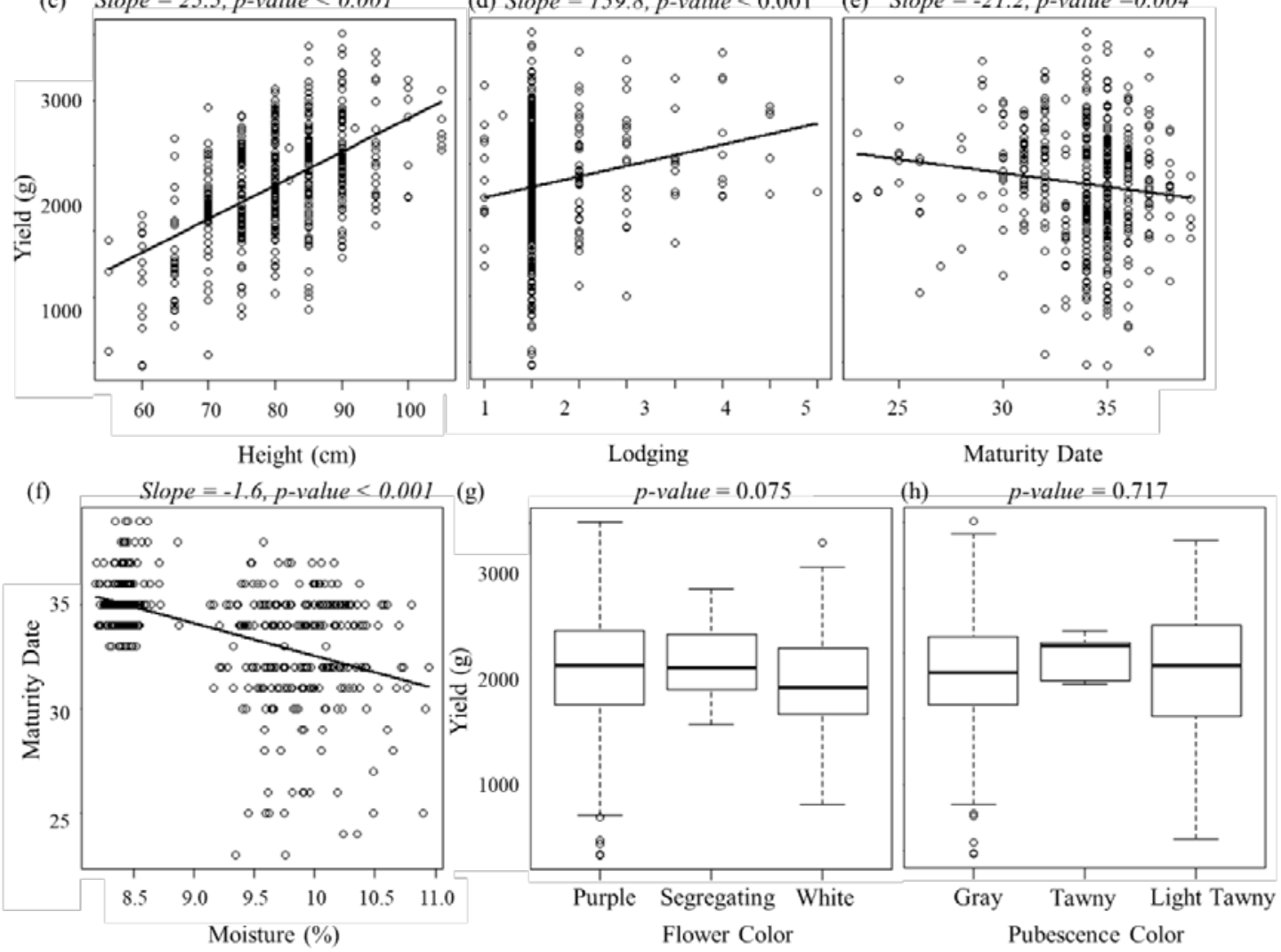

Figure 4-7. Manually measured agronomic traits in PYT. (a) are the visualization plots of the variables' contributions and directions in the plane of the first two PCs. (b) is the projection of sample variables on

the first two PCs. (c)-(e) are scatterplots of yield versus height, lodging and maturity date,

respectively. (f) is a scatterplot of the relationship between moisture and maturity date. (g)-(h) are boxplots of the yield of three groups of flower color and three groups of pubescence color. The $p$ value above (c)-(h) indicates the significance level of estimation.

\subsubsection{Differences in image features between selected and non-selected varieties}

Figure 4-8 shows the differences in 38 image features collected multiple times in the PT, PYT and AYT. Each square indicates the existence of a difference in the means of 
an image feature (all listed above) between the selected and non-selected groups. The DAPs for collecting images in each trial was on the left side. There were image features from PT, PYT and AYT from top to bottom. In the PT, there were 37, 35, 31 and 31 image features significantly different between the two groups, collected on DAP 75, 90, 108 and 120, respectively. There were 31, 32, 24, 4, 28 and 7 image features in the PYT and 34, 33, 28, 26, 18, 3 image features in the AYT that showed significant differences from the early to late stages. Among the 38 image features, 17 had consistently significant patterns (i.e., the selected group consistently higher or lower than the non-selected group) for the three trials.

The selected group has significantly higher values than the non-selected one

The selected group has significantly lower values than the non-selected one

No significant difference found between the selected and non-selected groups

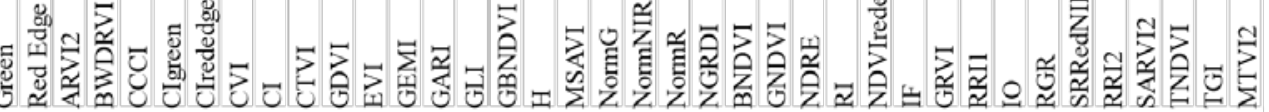
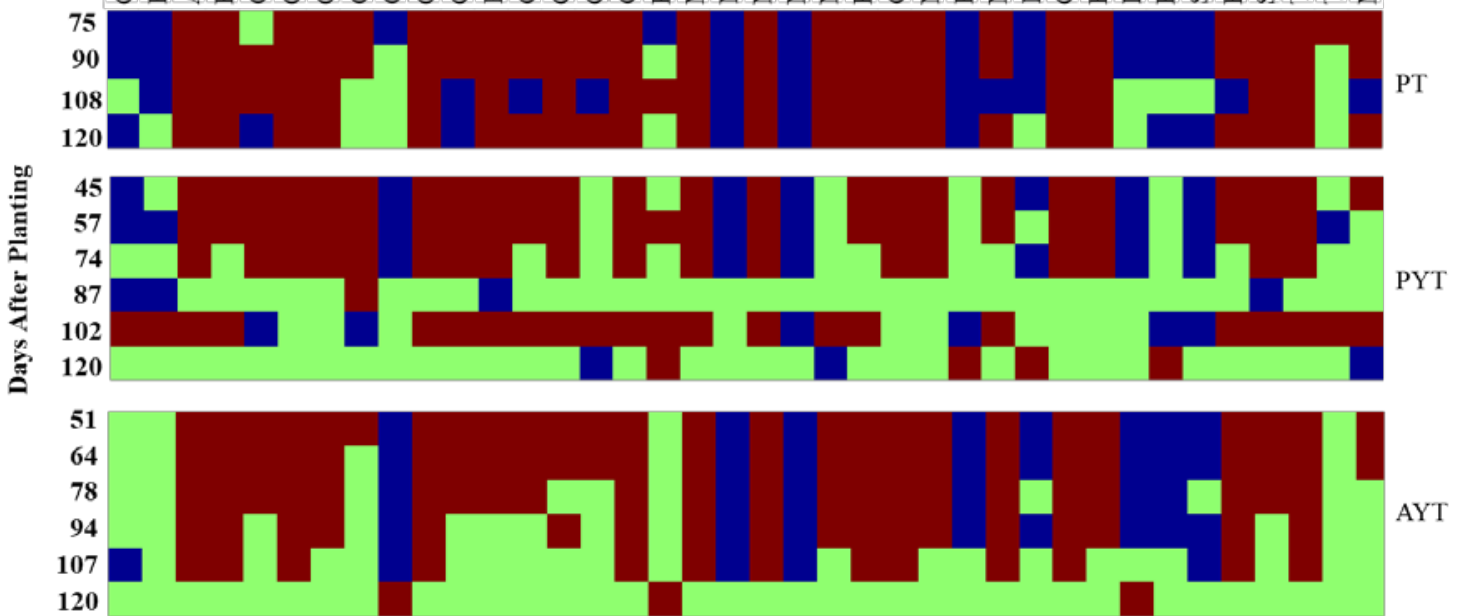

Figure 4-8. Illustration of the differences in image features between the selected and non-selected groups. Each square represents if there is a significant difference in an image feature collected at single times (p-value $<0.05$ ) between the two groups and which had higher values.

4.4.4. Profile analysis results in image features

Results of the profile analysis on the 38 image features show that all the image 
features in the PT displayed different patterns ( -value $<0.05)$ over time between the selected and non-selected groups, while 37 image features (except for IO) in the PYT and 31 features (except for red-edge, GLI, IF, RGR, and TGI) in the AYT had different patterns (p-value $<0.05$ ). Figure 4-9 shows the plots of GNDVI as an example of the profile analysis. The profile plots look parallel between the two groups due to the extended dynamic ranges over three years. A group of profile plots in Appendix Figure A1 was plotted with different GNDVI scales. It can be seen that the non-selected group usually had higher variations than the selected group. The variations of GNDVI collected at single times in the PT were higher than those in the PYT and AYT at similar times. In both PT and AYT, the nonselected group had a sharper increasing slope before the 90 DAP (around the R5 stage). The profiles showed that the 90 DAP was a changing point that the GNDVI values slowly increased before the stage and then started decreasing sharply.

It is noted that in the PYT, the GNDVI values had extremely low values among all data points. Appendix Figure A2 shows an RGB image and a GNDVI map collected at the 120 DAP for each of the three trials. It can be seen that the PT had a very large variation in the canopy greenness, i.e., maturity status. There were a number of lines have matured for some time (yellow and dry canopy), while most of the plots still had many green leaves. The PYT and AYT obviously had less vegetation than the PT and higher uniformity in maturity dates (mostly in 20-40 days after Sept. $1^{\text {st }}$ ). The GNDVI values for soil background in the three maps were consistently within a range of 0.3-0.45 and those for the light green leaves were around 0.6. However, the mature plots in the PYT contributed a lot to the low GNDVI values with some had similar values to the soil background. 

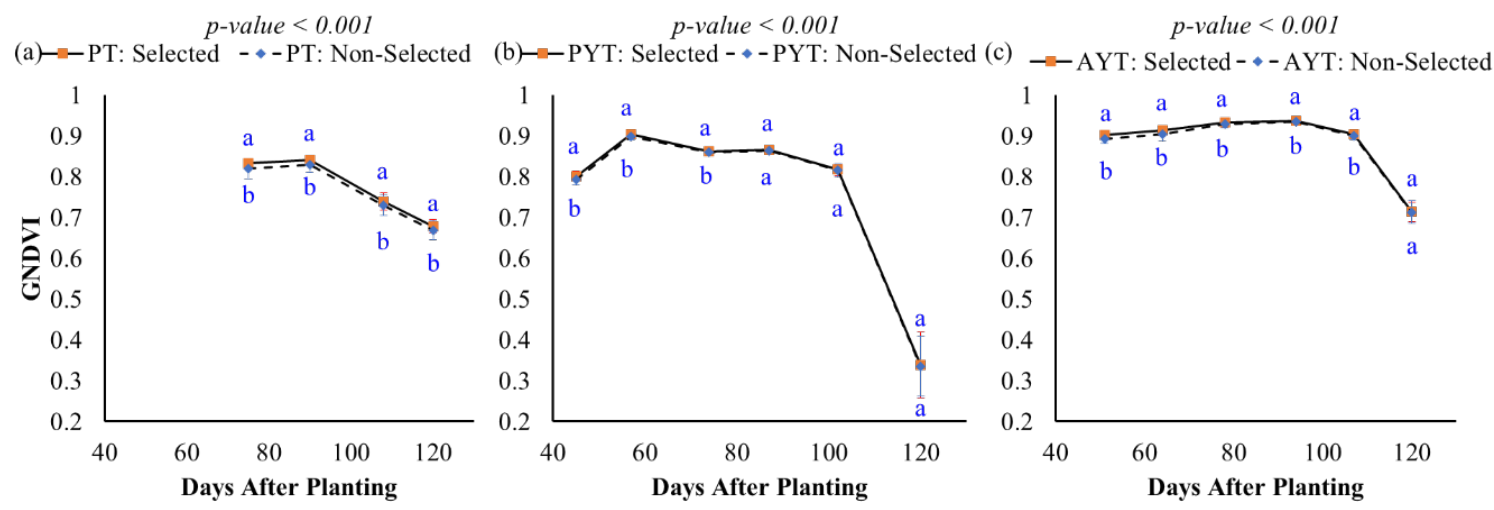

Figure 4-9. Profile plots of GNDVI for the selected and non-selected groups over the growing seasons. (a) - (c) are plots for the PT, PYT and AYT, respectively. The lower-case letters above bars indicate the significant difference among these means at a 0.05 significance level. The pvalue above each plot indicates the significance level of the null hypothesis that the two groups had a parallel pattern over time in GNDVI. Appendix Figure A1 shows the profile plots with different GNDVI scales.

\subsubsection{Performance of the model selections}

The performance of the model selections for the PT and PYT is shown in Figure 410. In all the breeder's selections of progeny rows, the part selected by the lasso model had a significantly higher yield in PYT than the non-selected part, when the model used image features collected at 75, 90 and 108 DAPs. No significance was observed when using the image features on the 120 DAP. However, the significance was not observed in yield from the AYT between the two groups. In the breeder's selection of the PYT lines, significant differences were observed in the PYT yield when using image features before the R5 stage (the 45, 57 and 74 DAP) and in AYT yield only at the 57 DAP. The numbers in blue show the classification recalls of model selections, representing the proportions of the breeder's selection that were also selected by the model. For both PT and PYT, the model was able to identify over $60 \%$ of the breeder's selections from the testing sets. The highest recalls were 0.71 (90 DAP) and 0.76 (102 DAP) for the PY and PYT, respectively.

Comparing the model selected part with all the lines of breeder's selections of progeny rows, yield in the PYT of the model selection (90 DAP) were up to $4 \%$ (106 g/plot) 
higher than the breeder's selection and 1\% (133/plot) higher in the AYT. While comparing for the selections of PYT lines, yield of the model selection (57 DAP) was up to 2\% (59 g/plot) higher than the breeder's selection in the PYT and 5\% (107/plot) higher in the AYT. It is noted that the highest recall $(0.76)$ in the PYT selections was obtained using image features on the 102 DAP, but the PYT yield of the model selection was not significantly higher than the remaining lines in the breeder's selection. On contrary, the AYT of the model selection was clearly lower than the remaining lines. A similar situation was observed for PT selections on the 120 DAP. Therefore, the high classification recall did not necessarily lead to higher yield performance in the following trials.

The significantly higher PYT yield of the model selections was achieved by the image features before the maturity stage (R8). Although there was no significant difference in the AYT yield in the corresponding DAP due to higher variations, the yield in model selections on the 75 (R4: the full pod stage) and 90 DAP (R5: the beginning of seed fill stage) was clearly higher. Similarly, the significance was observed in the PYT model selections before the 87 DAP (R5: the beginning of seed fill). Thus, image-based crop traits collected before maturity tended to perform better in selecting higher yielding progeny lines and, the appropriate time point for making selections for yield trials should be even earlier due to the less variation in these trials. 


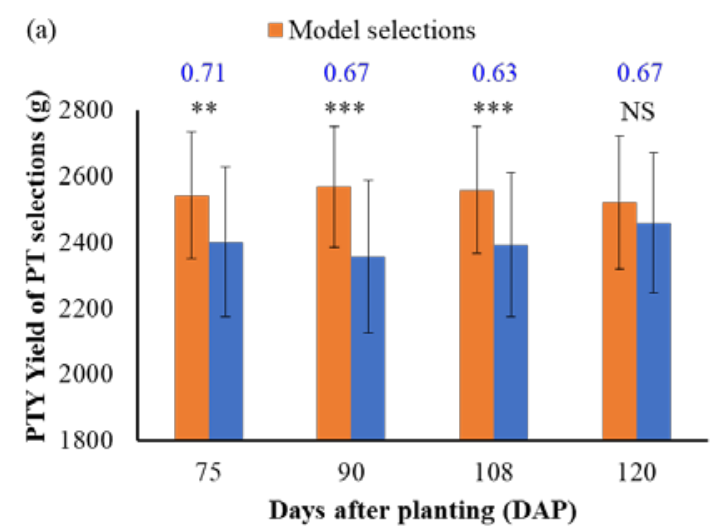

(b) Model Non-selections but selected by breeders
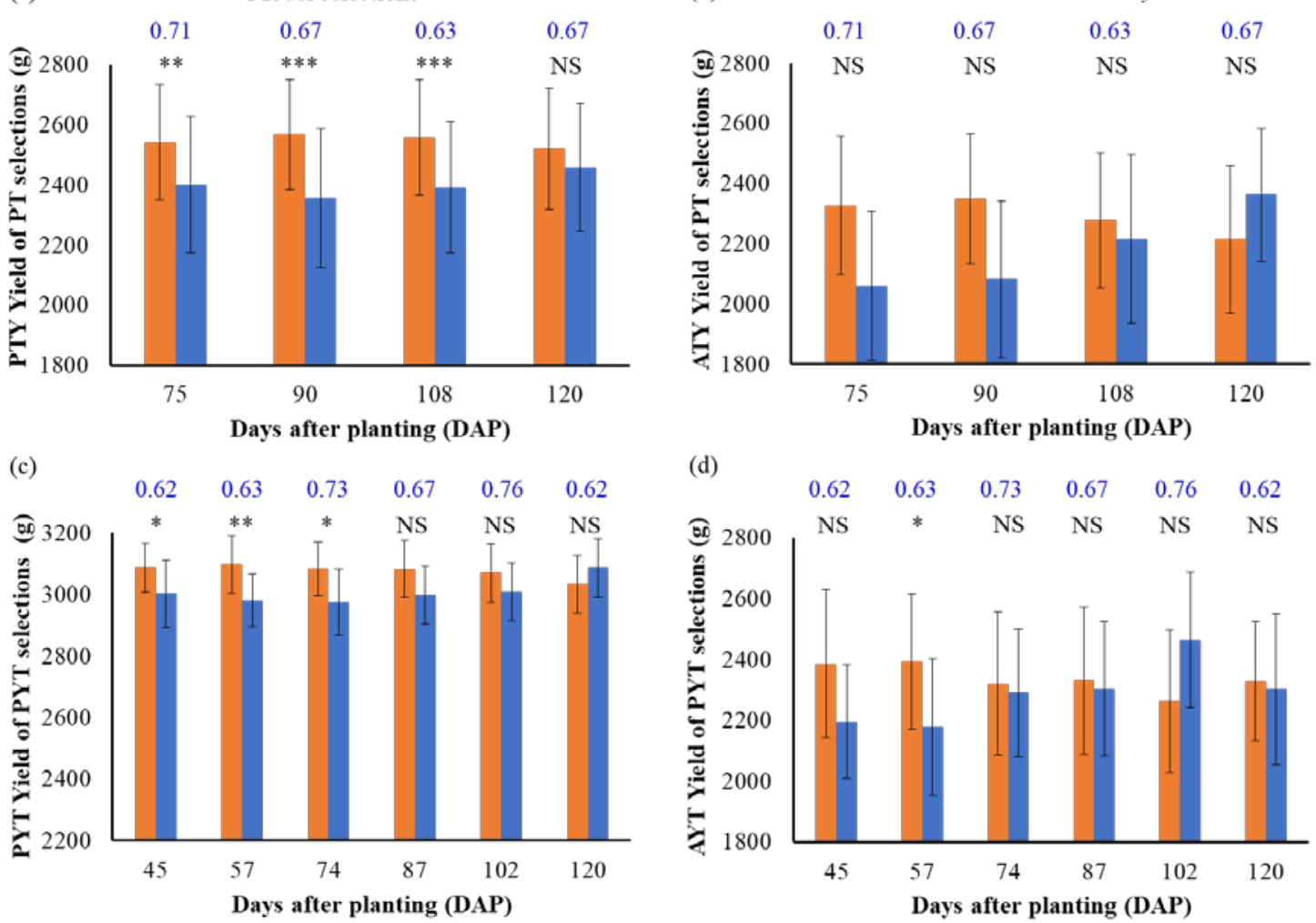

Figure 4-10. Model performance on selecting superior soybean lines from the PT and PYT. (a) and (c) Comparison of the PYT yield means. (b) and (d) Comparison of the AYT yield means. (a)-(b) are for the PT selections, while (c) and (d) are for the PYT selections. Orange and blue bars represent the model selections (the TP class) and model non-selections but selected by breeders (the FN class), respectively. Error bars show the standard deviations. *, **, *** indicate the significance levels of $p=0.05,0.01$, and 0.001 for testing the hypothesis that there is no difference in yield means between model selections and non-selections. NS represents no significance. The numbers in blue are the classification recall.

\subsection{Discussion}

Grain yield is the only trait that had significant differences (Figure 4-4a and 4-5a) in both yield trials, and it consistently and highly contributed (Figure 4-6a and 4-7a) to explain the variations in collected agronomic traits. As the most important trait for soybean breeding, yield has demonstrated its primary position among all the agronomic traits in this study. Yield estimation using remote sensing technologies has been a hot topic in the area of high-throughput phenotyping and yield has shown high correlations with image features 
(Herrmann et al., 2019; Maimaitijiang et al., 2020; Moreira et al., 2019). In one of our previous study (J. Zhou, J. Zhou, et al., 2021), the yield was highly correlated with VIs, such as GNDVI, and CCCI that were also shown to be significantly distinguishable for selecting superior soybean lines in all the three trials (Figure 4-8), implying the reliability of using these features make selections in scenarios without actually knowing yield, i.e., the PT.

Similar to yield, plant height is another agronomic trait that highly contributed to explaining the variations in both yield trials, which might be due to the significantly positive correlation between yield and height (Figure 4-6c and 4-7c). The selected group was taller than the non-selected group in the AYT, while with less variation and fewer outliers, there was no significant difference in plant height in the AYT. Compared to grain yield, plant height is easier to be measured using photographic methods with high accuracy and thus often used as an alternative to yield in agriculture studies. For example, Hassan et al. (2019) reported a significantly high correlation of $R^{2}=0.96$ with a $5.75 \mathrm{~cm}$ root mean square error between UAV-based plant height estimates and ground measurements in wheat, and Feng et al. (2019) reported a Pearson correlation coefficient of 0.96 between and cotton yield and UAV-based plant height.

It should be noted that the soybean materials in this study were indeterminate (IND) varieties that continue their vegetative growth and producing nodes on the main stem until the beginning of seed fill (R5) (Purcell et al., 2014). Compared to those with a determinate (DET) growth habit, i.e., stop vegetative growth and producing nodes before the flowering stages, IND soybeans usually have tall plants and averagely high yield, however, they are more susceptible to lodging. Height VS yield between indeterminate (IND) and determined 
soybean. It was reported by Wilcox and Sediyama (1981) that seed yield increased by 350 $\mathrm{kg} / \mathrm{ha}$ and lodging score increased by 0.008 for every $10 \mathrm{~cm}$ increase in plant height among the determinate lines, while seed yield increased by $112 \mathrm{~kg} / \mathrm{ha}$ and lodging score by 0.3 for every $10 \mathrm{~cm}$ increase in plant height among indeterminate lines. Therefore, plant lodging is nonnegligible in soybean breeding and those with severe lodging have to be avoided, especially among the IND varieties because of its tall plant height due to elongation of the main stem after flowering (Kato et al., 2015).

Severe lodging was not observed in this study. There were only six and seven plots having the lodging scores above 3 in the PYT and AYT, respectively. And there was not a consistently incremental or decremental relationship shown between lodging and yield (Figure 4-6d and 4-7d). It has been shown that lodging from R3 (beginning pod) to R5 (beginning seed) have the greatest impact on yield, while lodging before R2 (full flowering) and after R6 (full seed) does not significantly impact yield but lodging at the maturity stage can have negative impacts on harvestability (Koester et al., 2014). As the lodging scores were taken when soybeans reached their R8 stages, they did not necessarily reflect the negative effects on the yield of potential lodging in the middle of the growing seasons.

Figure. 4-8 gives a general idea of how differentiable the image features were between two groups over the growing seasons and how consistently the differences in multiple years and locations. Significant differences were observed in many image features between the selected and non-selected groups in the three trials. The PT had consistent differences from the reproductive to maturity stages. On the contrary, the PYT and AYT plots had less distinguishable features after the R5 stage. In soybean breeding, the selection 
on progeny row is usually based on the breeder's visual examinations. The plots with a similar appearance to the surrounding checks would be preferable and have desired performance regarding yield potential and maturity date. Therefore, the selected progeny rows are expected to have low variations, compared to all the materials planted in the PT. Yield of soybean plants is mainly formed at before their R5 stages, as the results of conjunctive effects of genotype and environments. The bloom (R1-R2) and pod stages (R3R4) are critical for gaining seed yield by determining the numbers of flowers, pods and beans, seed size and pod dry weight. Any stress at these stages could cause more yield reduction than at any other time. The model performance was benefited by the most significance in canopy coverage and colors displayed at these stages, rather than the appearances of yellow and dry stems at the maturity stages.

GNDVI was proved to be more sensitive to the variation of chlorophyll content in crops and highly correlated with nitrogen content in leaves (Gitelson \& Merzlyak, 1996). The profile analysis on GNDVI clearly exhibited how soybean physiology changes over time. Despite the extremely lower value collected at 120 DAP in the PYT, the profile patterns were very consistent for all three years. Moreover, it is straightforward to see image features collected at early stages were more powerful in splitting the selected and non-selected groups.

The selection confirms with the profile analysis that image features at early stages had better performance in selecting lines because the early model selections tended to have significantly higher productions in the next year, compared with the breeder's selections. Due to higher variations in the PT materials, superior progeny rows were easier to be identified with image features collected through reproductive stages (75-108 DAP). With 
the model selection, nearly $30 \%$ of soybean lines could be cut off in the following yield trials, consequently saving $30 \%$ of land usage, human labor and other resources. With the aid of efficient data collection and selection, human interactions could be eliminated a lot from the traditional breeding pipeline and the limitation on the number of progeny rows could be boosted. Hence the genetic gain could be increased by a higher selection intensity benefited from higher population size.

\subsection{Conclusion}

This study evaluated the breeder's criterion on selecting superior soybean for soybean breeding programs varieties using a group of agronomic traits and investigated the potential of making the selections using image-based secondary traits. A total of 11473 progeny rows were planted in 2018 (PT) and 1773 among them were selected for the preliminary yield trial (PYT) in 2019 and 238 were then selected for the advance yield trial (AYT) in 2020. Seven agronomic traits, including yield, plant height, maturity data, flower and pubescence color, moisture and lodging were manually measured for soybeans in the two yield trials. Unmanned Aerial Vehicle-based images were collected every two weeks over the growing seasons, and a group of image features was extracted from five-band multispectral images for each trial. Results show that yield is the primary trait for selecting superior soybean lines as there were significant differences in yield between the selected and non-selected group for both yield trials. Profile analysis on the image features showed that progeny rows had the most variation among three trials and the images collected before the beginning of seed fill stage (R5) explained more variation than those at later stages, consistently for the PT, PYT and AYT. The lasso model for selecting soybean lines with image features correctly identified $71 \%$ and $76 \%$ of the breeder's selection for the PT and 
PYT. The PYT yield in PT model selections before 120 DAP (the maturity stage R8) and the PYT model selection before 87 DAP (the beginning of seed fill stage, R5) was significantly higher $(p$-value $<0.05)$ than the model non-selections but selected by breeders. The model selections in PT and PYT had respectively 4\% and 5\% higher yield in the following year's trial, comparing the breeder's selection. It could be concluded that the proposed model is promising in making selections on soybean breeding trials.

\section{Reference}

Ahmar, S., Gill, R. A., Jung, K.-H., Faheem, A., Qasim, M. U., Mubeen, M., et al. (2020). Conventional and Molecular Techniques from Simple Breeding to Speed Breeding in Crop Plants: Recent Advances and Future Outlook. International journal of molecular sciences, 21(7), 2590. 10.3390/ijms21072590

Araus, J. L., \& Cairns, J. E. (2014). Field high-throughput phenotyping: the new crop breeding frontier. Trends in Plant Science, 19(1), 52-61

Araus, J. L., Kefauver, S. C., Zaman-Allah, M., Olsen, M. S., \& Cairns, J. E. (2018). Translating high-throughput phenotyping into genetic gain. Trends in Plant Science, 23(5), 451-466. https://doi.org/10.1016/j.tplants.2018.02.001

Arruda, M. P., Lipka, A. E., Brown, P. J., Krill, A. M., Thurber, C., Brown-Guedira, G., et al. (2016). Comparing genomic selection and marker-assisted selection for Fusarium head blight resistance in wheat (Triticum aestivum L.). Molecular Breeding, 36(7), 84. 10.1007/s11032-016-0508-5

Emlid. (2021). Reach RS/RS+ docs: Placing the base. Retrieved from https://docs.emlid.com/reachrs/common/tutorials/placing-the-base/

Fehr, W. R. (1991). Principles of cultivar development: theory and technique. New York, NY: Macmillian.

Feng, A., Zhang, M., Sudduth, K. A., Vories, E. D., \& Zhou, J. (2019). Cotton yield 
estimation from UAV-based plant height. Transactions of the ASABE, 62(2), 393-404. https://doi.org/10.13031/trans.13067

Furbank, R. T., \& Tester, M. (2011). Phenomics - technologies to relieve the phenotyping bottleneck. Trends in Plant Science, 16(12), 635-644. https://doi.org/10.1016/j.tplants.2011.09.005

Gitelson, A., \& Merzlyak, M. N. (1996). Signature analysis of leaf reflectance spectra: algorithm development for remote sensing of chlorophyll. Journal of Plant Physiology, 148(3-4), 494-500. https://doi.org/10.1016/S0176-1617(96)80284-7

Hassan, M. A., Yang, M., Fu, L., Rasheed, A., Zheng, B., Xia, X., et al. (2019). Accuracy assessment of plant height using an unmanned aerial vehicle for quantitative genomic analysis in bread wheat. Plant methods, 15(1), 37. 10.1186/s13007-0190419-7

Herrmann, I., Bdolach, E., Montekyo, Y., Rachmilevitch, S., Townsend, P. A., \& Karnieli, A. (2019). Assessment of maize yield and phenology by drone-mounted superspectral camera. Precision Agriculture, 21, 51-76. https://doi.org/10.1007/s11119-019-09659-5

Kato, S., Fujii, K., Yumoto, S., Ishimoto, M., Shiraiwa, T., Sayama, T., et al. (2015). Seed yield and its components of indeterminate and determinate lines in recombinant inbred lines of soybean. Breeding science, 65(2), 154-160. $10.1270 /$ jsbbs. 65.154

Koester, R. P., Skoneczka, J. A., Cary, T. R., Diers, B. W., \& Ainsworth, E. A. (2014). Historical gains in soybean (Glycine max Merr.) seed yield are driven by linear increases in light interception, energy conversion, and partitioning efficiencies. Journal of Experimental Botany, 65(12), 3311-3321. 10.1093/jxb/eru187

Maimaitijiang, M., Sagan, V., Sidike, P., Hartling, S., Esposito, F., \& Fritschi, F. B. (2020). Soybean yield prediction from UAV using multimodal data fusion and deep learning. Remote Sensing of Environment, 237, 111599. https://doi.org/10.1016/j.rse.2019.111599

Makanza, R., Zaman-Allah, M., Cairns, J. E., Eyre, J., Burgueño, J., Pacheco, A., et al. 
(2018). High-throughput method for ear phenotyping and kernel weight estimation in maize using ear digital imaging. Plant methods, 14(1). 10.1186/s13007-018-0317-4

Mammadov, J., Aggarwal, R., Buyyarapu, R., \& Kumpatla, S. (2012). SNP Markers and Their Impact on Plant Breeding. International Journal of Plant Genomics, 2012, 11. $10.1155 / 2012 / 728398$

Moreira, F. F., Hearst, A. A., Cherkauer, K. A., \& Rainey, K. M. (2019). Improving the efficiency of soybean breeding with high-throughput canopy phenotyping. Plant methods, 15(1), 139. https://doi.org/10.1186/s13007-019-0519-4

Orf, J. H., Diers, B. W., \& Boerma, H. R. (2004). Genetic improvement: Conventional and molecular-based strategies. Soybeans: Improvement, production, and uses, $16,417-450$

Purcell, L. C., Salmeron, M., \& Ashlock, L. (2014). Soybean growth and development. Arkansas Soybean Production Handbook, MP197, 1-8

Richards, R. (2000). Selectable traits to increase crop photosynthesis and yield of grain crops. Journal of Experimental Botany, 51(suppl_1), 447-458

Sleper, D. A., \& Poehlman, J. M. (2006). Breeding field crops: Blackwell publishing.

Watanabe, K., Guo, W., Arai, K., Takanashi, H., Kajiya-Kanegae, H., Kobayashi, M., et al. (2017). High-throughput phenotyping of sorghum plant height using an unmanned aerial vehicle and its application to genomic prediction modeling. Frontiers in Plant Science, 8. 10.3389/fpls.2017.00421

Wilcox, J. R., \& Sediyama, T. (1981). Interrelationships among height, lodging and yield in determinate and indeterminate soybeans. Euphytica, 30(2), 323-326. 10.1007/BF00033993

Xu, Y., Li, P., Zou, C., Lu, Y., Xie, C., Zhang, X., et al. (2017). Enhancing genetic gain in the era of molecular breeding. Journal of Experimental Botany, 68(11), 26412666. $10.1093 / \mathrm{jxb} / \mathrm{erx} 135$

Zhang, C., Craine, W., Davis, J. B., Khot, L. R., Marzougui, A., Brown, J., et al. (2018). 
Detection of canola flowering using proximal and aerial remote sensing techniques. Paper presented at the Proceedings of SPIE - The International Society for Optical Engineering. 10.1117/12.2304054

Zhou, J., Mou, H., Zhou, J., Ali, M. L., Ye, H., Chen, P., et al. (2021). Qualification of soybean responses to flooding stress using UAV-based imagery and deep learning. Manuscript under review

Zhou, J., Yungbluth, D., Vong, C. N., Scaboo, A., \& Zhou, J. (2019). Estimation of the maturity date of soybean breeding lines using UAV-based multispectral imagery. Remote Sensing, 11(18), 2075. https://doi.org/10.3390/rs11182075

Zhou, J., Zhou, J., Ye, H., Ali, M. L., Chen, P., \& Nguyen, H. T. (2021). Yield estimation of soybean breeding lines under drought stress using unmanned aerial vehiclebased imagery and convolutional neural network. Biosystems Engineering, 204, 90-103. https://doi.org/10.1016/j.biosystemseng.2021.01.017

Zhou, J., Zhou, J., Ye, H., Ali, M. L., Nguyen, H. T., \& Chen, P. (2020). Classification of soybean leaf wilting due to drought stress using UAV-based imagery. Computers and Electronics in Agriculture, 175, 105576. https://doi.org/10.1016/j.compag.2020.105576

Zhou, S., Mou, H., Zhou, J., Zhou, J., Ye, H., \& Nguyen, H. T. (2021). Development of an automated plant phenotyping system for evaluation of salt tolerance in soybean. Computers and Electronics in Agriculture, 182, 106001. https://doi.org/10.1016/j.compag.2021.106001 


\section{CHAPTER FIVE}

\section{CONCLUSIONS AND FUTURE WORK}

In this research, an integrated and automated UAV HTP platform was developed to deliver image-based crop traits for soybean breeding programs. It was equipped with multispectral and digital cameras to take images with high spatial and temporal resolutions, as well as GNSS units to provide precise geo-referencing for image stitching and processing. Images collected at different days were calibrated to eliminate the effects of light variations on image pixel numbers and thus ensure consistent spectral reflectance. An automated pipeline was developed to process the time-series images and extract a number of image features for each soybean plot. To evaluate the potential of improving breeding efficiency using the UAV-based HTP platform and pipeline, a typic soybean breeding program was traced for three years from the progeny trial to the preliminary yield trials and then to the advanced yield trials. Two key agronomic traits, i.e., maturity date and yield were estimated using image features and machine learning models. A group of elite soybean genotypes was selected by learning from the breeder's selections. The performance of the proposed framework was evaluated by comparing the image estimates with the manually measured traits. Detailed conclusions are as follows.

\subsection{Conclusions}

The analysis on image features and estimation of soybean maturity dates showed that that the best agreement of predicted maturity dates and manual measurements was made by image features collected roughly when soybean was at the beginning maturity status, i.e., starting to have fallen leaves and yellow pods. It was found that four image features (CIrededge_mean, GDVI_std, GRVI_mean and BNDVI_mean) had positive 
linear relationships with the maturity dates, and five image features (S_mean, CVI_std, IF_std, CI_mean and IF_mean) were negatively related to the maturity dates. The adjusted maturity dates proposed in this study were able to tolerate the variances caused by the conjunction of genetic and environmental variances. The maturity dates were predicted using a partial least square regression (PLSR) model and the results showed that the image predicted maturity date highly agreed $\left(R^{2}=0.81\right)$ with the visually rated value and the root mean square error (RMSE) of 1.4 days. By personal communication with breeders, RMSE within 1.5 days can be considered as a tolerable error in soybean breeding programs. Compared to previous studies, the proposed methods showed improvements in predicting the exact maturity dates instead of the classification between mature and immature lines, as well as making a more practical prediction for breeding programs.

A CNN model was developed to estimate soybean yield taken seven image features representing plant height, canopy color, and canopy texture as the predictors. The analysis on the RGB and multispectral image features acquired at three growth stages showed that the image features collected at the early and late reproductive growth stages were comparably promising in estimating soybean yield. It was also observed that high-yield soybean lines had a darker green color with a full and wide canopy shape, a higher plant height, and a more uniform canopy texture. As the breeding materials were in three maturity group and under drought stress, two categorical traits, i.e. maturity groups (III, IV and V) and drought tolerance (fast- and slow-wilting) were also included in a mixed version of the CNN model. The improved prediction model could explain 78\% of the measured yield with a root mean square error of $391.0 \mathrm{~kg} \cdot \mathrm{ha}^{-1}$ (33.8 \% to average yield).

To model the breeder's selection criteria, seven agronomic traits, including yield, 
plant height, maturity data, flower and pubescence color, moisture and lodging were manually measured for soybeans in two yield trials. The principal component analysis (PCA) showed that yield is the primary trait for selecting superior soybean lines as there were significant differences in yield between the selected and non-selected groups for both yield trials. In addition, plant height is another agronomic trait that highly contributed to explaining the variations in both yield trials due to the significantly positive correlation between yield and height. It was noted that for indeterminate (IND) materials (the kind that used in this soybean in this study, plant lodging is a nonnegligible factor for selecting elite genotypes and those with severe lodging have to be avoided. The profile analysis showed that image features at early stages had better performance in selecting lines as soybean plants at early stages exhibited larger variances in their appearances. Due to higher variations in the PT materials, superior progeny rows were easier to be identified with image features collected through early reproductive stages (75-108 DAP). compared the yield trials. A Lasso model for selecting soybean lines with image features correctly identified $71 \%$ and $76 \%$ of the breeder's selection for the PT and PYT. The model selections in PT and PYT had respectively $4 \%$ and 5\% higher yield, comparing the breeder's selection.

The developed UAV HTP platform is able to efficiently collect multi-dimensional images on soybean breeding materials, for example, it can cover a 9-acres breeding field within 2 hours. The automated pipeline can generate tabulated image features for each soybean plot within 24-48 hours after data collection. For images collected multiple times in a field, the pipeline can segment soybean plots and output their image features from orthomosaic images efficiently within 10 min for over 10,000 plots, leveraging the precise 
georeferencing information. By comparing with manual/ground data, the accuracy of estimating the maturity date and yield using image features and machine learning models is sufficient for breeding purposes. The proposed framework largely increases the efficiency of scouting the fields and measuring plant performances for breeders, thus breaking the constraints of the numbers of breeding lines in a trial due to limited human labors. Breeding efficiency would be improved by the higher probability to identify elite lines as more materials could be tested in a trial. The model selections of elite lines confirmed near $70 \%$ of the breeder's selections and their intersections had better yield performance than the remaining. The HTP framework can successfully assist breeders to identify lines with better yield performance and select a smaller group than the regular percentages. Therefore it helps to increase the selection intensity and eventually genetic gain. Moreover, with a smaller group of candidates, land usage, human labor and other resources will be saved correspondingly, allowing breeding resources to be relocated for more efficient strategies. With all respects, it is concluded that the proposed framework is promising in improving the soybean breeding efficiency by increasing the selection intensity and accuracy, and saving resources.

\subsection{Future work}

Based on the observations and results from this research, future work should focus on: (1) There were two major issues expected to be addressed to reduce errors introduced during the image collection and processing pipeline. First, as an image pixel value is the reflectance of incoming solar irradiance, the variation in light conditions could introduce variations to image-derived features. The relationship between light changes during UAV flight missions and camera responses (reflectance) should be further explored and flight 
dynamics (drone pose, sun's position, light diffusion, clouds, etc.) need to be better integrated to describe the light changes. Second, variations are inevitably introduced when images are stitched based on feature recognition. Instead of localizing individual breeding lines from an orthomosaic, efforts have to be made to investigate the potential of direct georeferencing that the geometric position of a breeding line is the conjunction of the relative position of the line in an image and the UAV position where the image was taken. With direct georeferencing, flight attitude and speed can be liberated from high image overlaps ( $\geq 70 \%$ for creating orthomosaic images) so that the time issue and light variation can be moderated consequently.

(2) The proposed models for yield estimation and maturity date should be further validated by repeating the experiments in multiple environments and documenting image features. To make the model parameters robust and universal for other soybean breeding programs, soybean materials with more genetic variations should be added to the training dataset by collecting both UAV-based images and ground data. Moreover, the models could be further improved by adding variables related to environmental factors, such as soil conditions, temperature, and precipitation, etc.

(3) The image-based model selections of soybean elite progeny rows should be further evaluated by side-by-side comparisons of the yield-potential in following yield trials between all the model selections and the breeder's selections. Other than solely trained by the breeder selections, the model would learn to process other information from the provided image features, i.e., estimating each of the factors that are critical to a specific breeding program, such as yield, lodging and maturity dates, etc., and selectively integrating these factors to make selections. 


\section{APPENDIX}

Table A1. Vegetation indices.

\begin{tabular}{|c|c|c|c|}
\hline No. & Index Name & Descriptions & Formula \\
\hline \multirow{3}{*}{1} & \multirow{2}{*}{ NDVI } & Normalized & \multirow{4}{*}{$1.22 \times \frac{\frac{\text { nir }- \text { red }}{\text { nir }+ \text { red }}}{1.22 * \text { nir }+ \text { red }-0.0366+0.08 \times\left(1+a^{2}\right)}$} \\
\hline & & difference VI* & \\
\hline & & $\begin{array}{c}\text { Adjusted } \\
\text { transformed soil-- }\end{array}$ & \\
\hline 2 & ATSAVI & $\begin{array}{l}\text { transformed soll- } \\
\text { adjusted VI }\end{array}$ & \\
\hline 3 & ARVI2 & $\begin{array}{l}\text { Atmospherically } \\
\text { Resistant VI } 2\end{array}$ & $-0.18+0.17 \times \frac{\text { nir }- \text { red }}{\text { nir }+ \text { red }}$ \\
\hline 4 & BWDRVI & $\begin{array}{l}\text { Blue-wide dynamic } \\
\text { range VI }\end{array}$ & $\frac{0.1 \times \text { nir }- \text { red }}{0.1 \times n i r+r e d}$ \\
\hline 5 & CCCI & $\begin{array}{l}\text { Canopy } \\
\text { Chlorophyll } \\
\text { Content Index }\end{array}$ & $\frac{\text { nir }-r e}{\text { nir }+r e}$ \\
\hline 6 & CIgreen & Chlorophyll Index & nir \\
\hline 7 & CIrededge & $\begin{array}{l}\text { Green } \\
\text { Chlorophyll Index } \\
\text { RedEdge }\end{array}$ & $\begin{array}{l}\text { green }-1 \\
\frac{\text { nir }}{r e-1}\end{array}$ \\
\hline 8 & CVI & Chlorophyll VI & $\begin{array}{c}\text { re }-1 \\
\text { nir } \times \frac{\text { red }}{\text { green }^{2}}\end{array}$ \\
\hline 9 & CI & Coloration Index & $\frac{\text { red }- \text { blue }}{\text { red }}$ \\
\hline 10 & CTVI & $\begin{array}{l}\text { Corrected } \\
\text { Transformed VI }\end{array}$ & $\frac{N D V I+0.5}{|N D V I+0.5|} \times \sqrt{|N D V I+0.5|}$ \\
\hline 11 & GDVI & $\begin{array}{l}\text { Green Difference } \\
\text { VI }\end{array}$ & nir - green \\
\hline 12 & EVI & Enhanced VI & $2.5 \times \overline{\text { nir }+6 \times \text { rod }-75 \times h l u+1}$ \\
\hline 13 & EVI2 & Enhanced VI 2 & $\begin{array}{c}\text { nir }+6 \times \text { red }-7.5 \times \text { blue }+1 \\
2.4 \times \frac{\text { nir }- \text { red }}{\text { nir }+ \text { red }+1} \\
\text { nir }- \text { red }\end{array}$ \\
\hline 14 & EVI22 & Enhanced VI 2-2 & $\begin{array}{c}2.5 \times \frac{n i r+2.4 \times r e d+1}{n i r}+0.5 \times r e d \\
2 \times\left(\text { nir }^{2}-\text { red }^{2}\right)+1.5 \times \text { nir }+0.5\end{array}$ \\
\hline 15 & GEMI & $\begin{array}{l}\text { Environment } \\
\text { Monitoring Index }\end{array}$ & $\begin{array}{l}a=\frac{\text { nir }+ \text { red }+0.5}{\text { GEMI }=a \times(1-0.25 \times a)-\frac{\text { red }-125}{1-\text { red }}}\end{array}$ \\
\hline 16 & GARI & $\begin{array}{l}\text { Green } \\
\text { atmospherically } \\
\text { resistant VI }\end{array}$ & $\frac{\text { nir }-(\text { green }-(\text { blue }- \text { red }))}{\text { nir }+(\text { green }+(\text { blue }- \text { red }))}$ \\
\hline 17 & GLI & Green leaf index & $2 \times$ green - red - blue \\
\hline 18 & GBNDVI & Green-Blue NDVI & $\begin{array}{c}2 \times \text { green }+ \text { red }+ \text { blue } \\
\text { nir }-(\text { green }+ \text { blue })\end{array}$ \\
\hline 19 & GRNDVI & Green-Red NDVI & $\begin{array}{l}\text { nir }+(\text { green }+ \text { blue }) \\
\text { nir }-(\text { green }+ \text { red }) \\
\end{array}$ \\
\hline & GIIVDV & & $\overline{\text { nir }+(\text { green }+ \text { red })}$ \\
\hline 20 & $\mathrm{H}$ & Hue & $\tan ^{-1}\left(\frac{2 \times \text { red }- \text { green }- \text { blue }}{30.5} \times(\right.$ green - blue $)$ \\
\hline 21 & IPVI & $\begin{array}{l}\text { Infrared percentage } \\
\text { VI }\end{array}$ & $\frac{n i r}{2 \times(n i r+r e d)} \times N D V I$ \\
\hline
\end{tabular}




\begin{tabular}{|c|c|c|c|}
\hline רי ד & & $J^{\prime}$ & red + green + blue \\
\hline 22 & 1 & Intensity & $\begin{array}{l}30.5 \\
\end{array}$ \\
\hline 23 & $\log R$ & Log Ratio & $\log \left(\frac{\text { nir }}{\text { red }}\right)$ \\
\hline 24 & MSAVI & $\begin{array}{l}\text { Modified Soil } \\
\text { Adjusted VI }\end{array}$ & $\frac{1}{2} \times\left(2 \times\right.$ nir $\left.+1-\sqrt{(2 \times \text { nir }+1)^{2}-8 \times(\text { nir }- \text { red })}\right)$ \\
\hline 25 & NormG & Norm Green & $\frac{\text { green }}{\text { nir }+ \text { red }+ \text { green }}$ \\
\hline 26 & NormNIR & Norm NIR & $\frac{\text { nir }}{\text { nir }+ \text { red }+ \text { green }}$ \\
\hline 27 & NormR & Norm Red & $\frac{\text { red }}{\text { nir }+ \text { red }+ \text { green }}$ \\
\hline 28 & NGRDI & $\begin{array}{l}\text { Normalized green } \\
\text { red difference index }\end{array}$ & $\frac{\text { green }- \text { red }}{\text { green }+ \text { red }}$ \\
\hline 29 & BNDVI & $\begin{array}{l}\text { Blue-normalized } \\
\text { difference VI }\end{array}$ & $\frac{\text { nir }- \text { blue }}{\text { nir }+ \text { blue }}$ \\
\hline 30 & GNDVI & Green NDVI & $\frac{\text { nir }- \text { green }}{\text { nir }+ \text { green }}$ \\
\hline 31 & NDRE & $\begin{array}{l}\text { Normalized } \\
\text { Difference Red- } \\
\text { Edge }\end{array}$ & $\frac{n i r-r e}{n i r+r e}$ \\
\hline 32 & RI & Redness Index & $\frac{\text { red }- \text { green }}{\text { red }+ \text { green }}$ \\
\hline 33 & NDVIrededge & $\begin{array}{l}\text { Normalized } \\
\text { Difference } \\
\text { Rededge/Red }\end{array}$ & $\frac{r e-r e d}{r e+r e d}$ \\
\hline 34 & PNDVI & Pan NDVI & $\frac{\text { nir }-(\text { green }+ \text { red }+ \text { blue })}{\text { nir }+(\text { green }+ \text { red }+ \text { blue })}$ \\
\hline 35 & RBNDVI & Red-Blue NDVI & $\frac{\text { nir }-(\text { red }+ \text { blue })}{\text { nir }+(\text { red }+ \text { blue })}$ \\
\hline 36 & IF & Shape Index & $\frac{2 \times \text { red }- \text { green }- \text { blue }}{\text { green }- \text { blue }}$ \\
\hline 37 & GRVI & Green Ratio VI & $\begin{array}{l}\text { green } \\
\text { nir }\end{array}$ \\
\hline 38 & DVI & Difference VI & $\frac{n t r}{r e d}$ \\
\hline 39 & RRI1 & $\begin{array}{l}\text { RedEdge Ratio } \\
\text { Index } 1\end{array}$ & $\frac{\text { nir }}{r e}$ \\
\hline 40 & IO & Iron Oxide & $\frac{\text { red }}{\text { blue }}$ \\
\hline 41 & RGR & Red-Green Ratio & $\overline{\text { green }}$ \\
\hline 42 & SRRedNIR & Red/NIR Ratio VI & $\frac{\text { red }}{\text { nir }}$ \\
\hline 43 & RRI2 & $\begin{array}{l}\text { Rededge/Red } \\
\text { RedEdge Ratio } \\
\quad \text { Index } 2\end{array}$ & $\frac{r e}{\text { red }}$ \\
\hline 44 & SQRTIRR & SQRT(IR/R) & $\sqrt{\frac{\text { nir }}{r e d}}$ \\
\hline 45 & TNDVI & Transformed NDVI & $\sqrt{\frac{\text { nir }- \text { red }}{\text { nir }+ \text { red }}}+0.5$ \\
\hline
\end{tabular}




\begin{tabular}{|c|c|c|c|}
\hline 46 & TGI & $\begin{array}{l}\text { Triangular } \\
\text { greenness index }\end{array}$ & $-0.5 \times(0.19 \times($ red - green $)-0.12 \times($ red - blue $))$ \\
\hline 47 & WDRVI & $\begin{array}{l}\text { Wide Dynamic } \\
\text { Range VI }\end{array}$ & $\frac{0.1 \times \text { nir }- \text { red }}{0.1 \times \text { nir }+ \text { red }}$ \\
\hline 48 & MSR & $\begin{array}{l}\text { Modified Simple } \\
\text { Ratio }\end{array}$ & $\frac{r e a}{\sqrt{\frac{\text { nir }}{\text { red }}+1}}$ \\
\hline 49 & МТУИ & Modified & $1.5 \times(1.2 \times($ nir - green $)-2.5 \times($ red - green $))$ \\
\hline 49 & MIVIL & Triangular VI & $\sqrt{(3-n i r)^{2}-6 \times n i r+5 \times \sqrt{r e d}-0.5}$ \\
\hline 50 & RDVI & $\begin{array}{l}\text { Renormalized } \\
\text { Difference VI }\end{array}$ & $\frac{\text { nir }- \text { red }}{\sqrt{\text { nir }+ \text { red }}}$ \\
\hline 51 & IRG & $\begin{array}{l}\text { Red Green Ratio } \\
\text { Index }\end{array}$ & red-green \\
\hline 52 & OSAVI & $\begin{array}{l}\text { Optimized Soil } \\
\text { Adjusted VI } \\
\text { Simple Ratio } \times\end{array}$ & $\frac{n i r-r e d}{n i r+r e d+0.16}$ \\
\hline 53 & SRNDVI & $\begin{array}{c}\text { Normalized } \\
\text { Difference } \\
\text { Vegetation Index }\end{array}$ & $\frac{n i r^{2}-r e d}{n i r+r e d^{2}}$ \\
\hline 54 & SARVI2 & $\begin{array}{c}\text { Soil and } \\
\text { Atmospherically } \\
\text { Resistant } \\
\text { Vegetation Index 2 }\end{array}$ & $2.5 \times \frac{\text { nir }- \text { red }}{1+\text { nir }+6 \times \text { red }-7.5 \times \text { blue }}$ \\
\hline
\end{tabular}

*VI: Vegetation index.
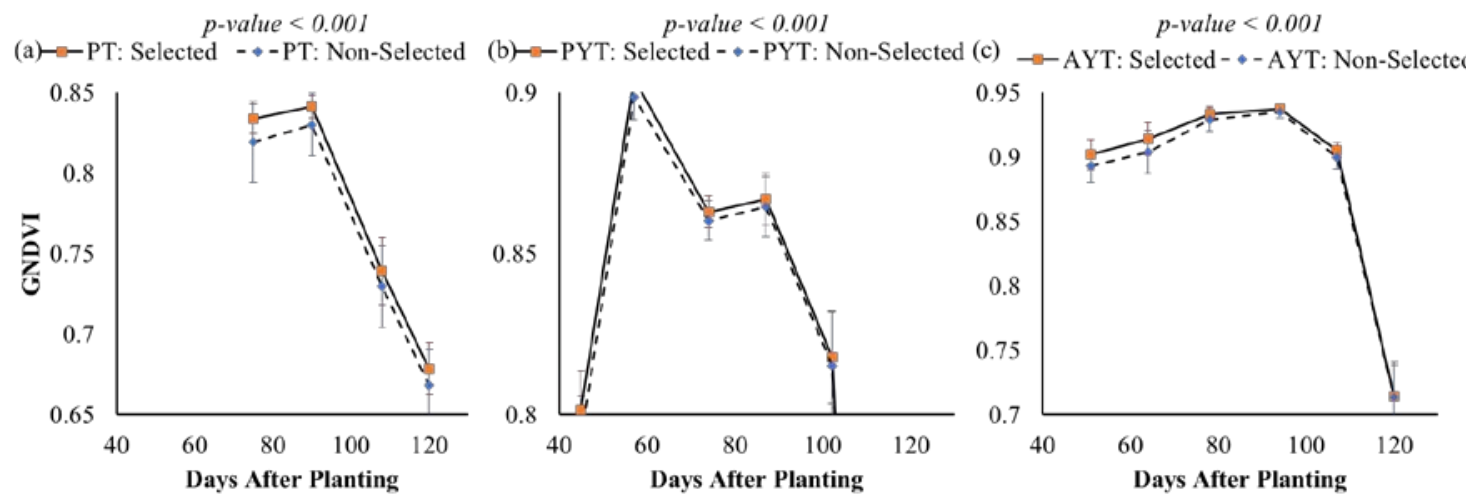

Figure A1. Profile plots of GNDVI for the selected and non-selected groups over the growing seasons. (a) - (c) are plots for the PT, PYT and AYT, respectively. They were plotted with different GNDVI scales to show the differences in profile patterns. The error bars represent the standard deviation. The p-value above each plot indicates the significance level of the null hypothesis that the two groups had a parallel pattern over time in GNDVI. 

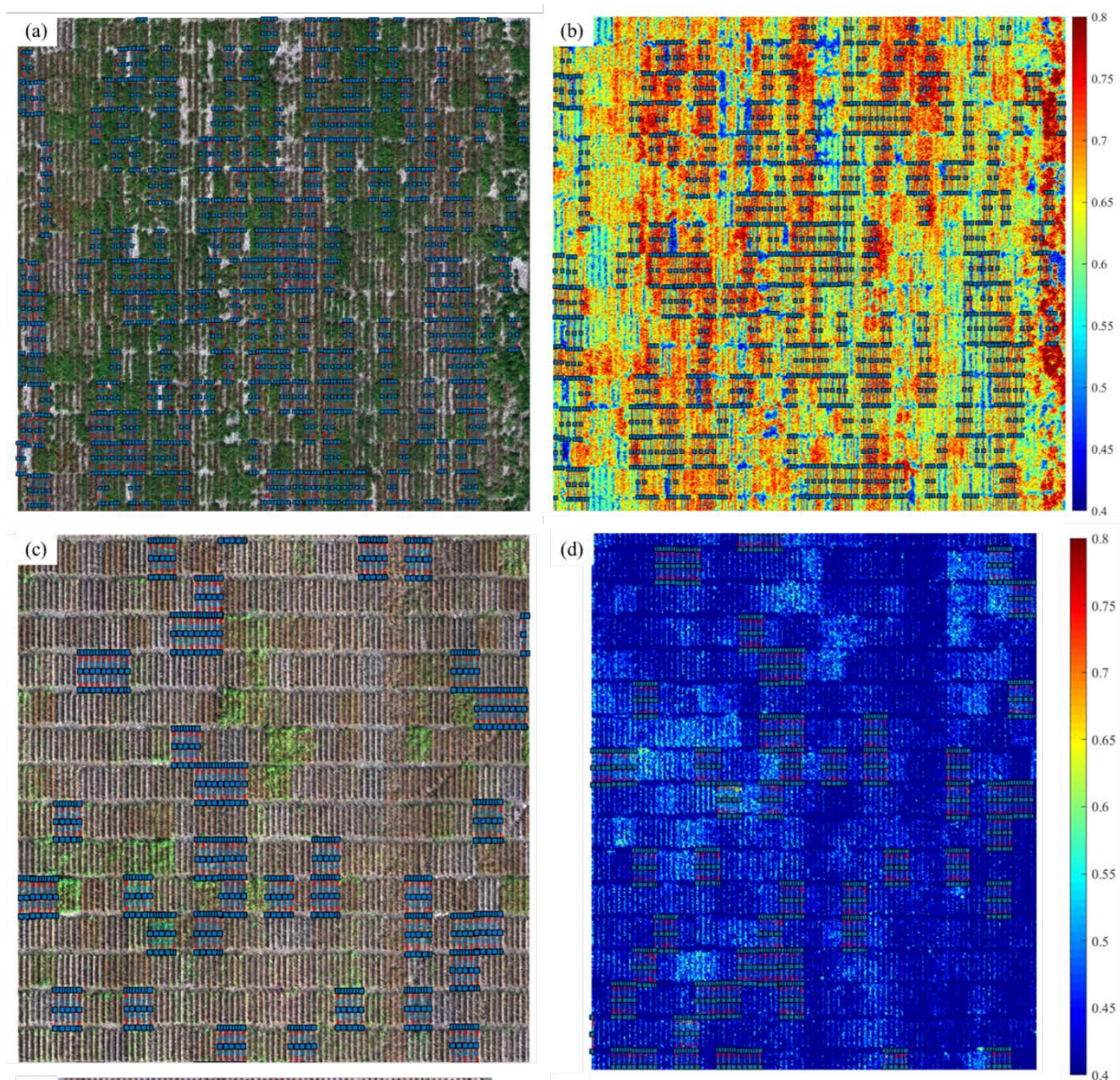

(e)

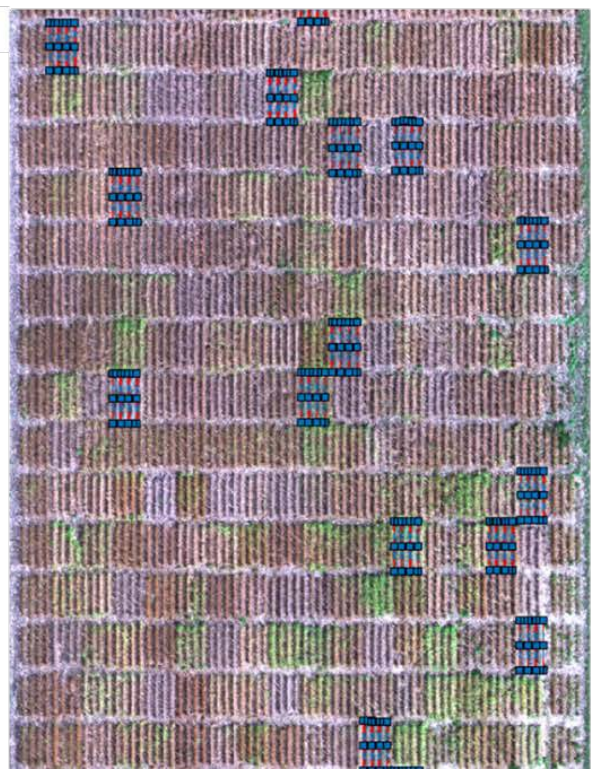

(f)

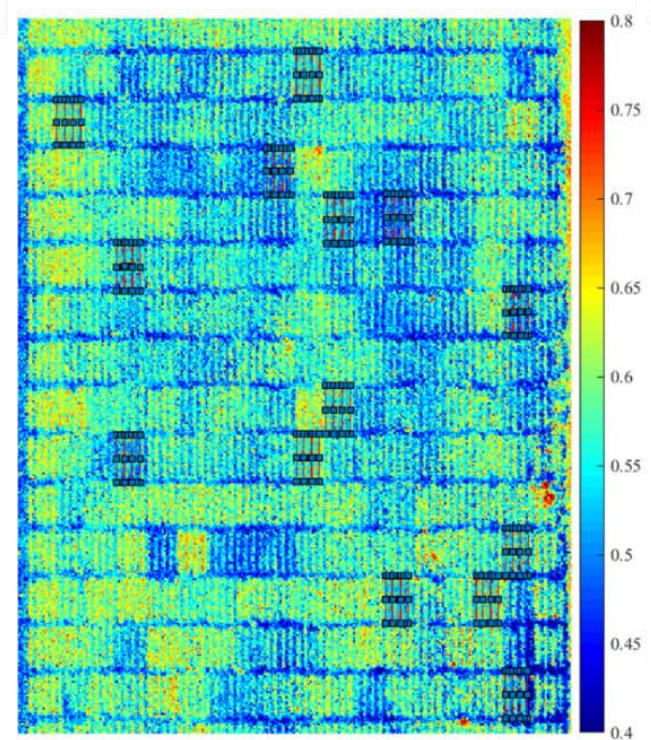


Figure A2. RGB images of the partial experimental fields in the three trials. The blue rectangles mark the selected plots. (a) and (b) were from the PT. (c) and (d) were from the PYT. (e) and (f) were from the AYT. (a), (c) and (e) are RGB bands of the multispectral images. (b), (d) and (f) were in the GNDVI. Images were collected on the 120 DAP for all three trials. 
Jing Zhou was born in April 1991 in Rugao, Jiangsu, China. He received his B.S. degree in Agricultural mechanization and its automation in 2013 and his M.E. in Agricultural Engineering in 2015 from Nanjing Agricultural University, Nanjing, Jiangsu, China. He received a Ph.D. degree major in Biological Engineering and a minor in Statistics from the University of Missouri in 2021. He was trained as an engineer with a multidisciplinary background in Mechanical and Electronical Engineering, Computer Engineering, Statistics, and plant science. His research interests focus on addressing agricultural challenges using modern technologies. 